Florida International University FIU Digital Commons

\title{
Human Papillomavirus Infection and Vaccination Policies in the American South
}

Dudith Pierre-Victor

Florida International University, dpier014@fiu.edu

DOI: $10.25148 /$ etd.FIDC000732

Follow this and additional works at: https://digitalcommons.fiu.edu/etd

Part of the Medicine and Health Sciences Commons

\section{Recommended Citation}

Pierre-Victor, Dudith, "Human Papillomavirus Infection and Vaccination Policies in the American South" (2016). FIU Electronic Theses and Dissertations. 2591.

https://digitalcommons.fiu.edu/etd/2591

This work is brought to you for free and open access by the University Graduate School at FIU Digital Commons. It has been accepted for inclusion in FIU Electronic Theses and Dissertations by an authorized administrator of FIU Digital Commons. For more information, please contact dcc@fiu.edu. 


\section{FLORIDA INTERNATIONAL UNIVERSITY}

Miami, Florida

\section{HUMAN PAPILLOMAVIRUS INFECTION AND VACCINATION POLICIES IN THE AMERICAN SOUTH}

A dissertation submitted in partial fulfillment of

the requirements for the degree of

DOCTOR OF PHILOSOPHY

in

PUBLIC HEALTH

by

Dudith Pierre-Victor

2016 
To: Dean Tomás R. Guilarte

R.Stempel College of Public Health and Social Work

This dissertation, written by Dudith Pierre-Victor, and entitled Human Papillomavirus Infection and Vaccination Policies in the American South, having been approved in respect to style and intellectual content, is referred to you for judgment.

We have read this dissertation and recommend that it be approved.

Mary Jo Trepka

$\begin{array}{r}\hline \text { Tan Li } \\ \hline \text { Timothy F. Page } \\ \hline \text { Dionne P. Stephens } \\ \hline \text { Purnima Madhivanan, Major Professor }\end{array}$

Date of Defense: June 6, 2016

The dissertation of Dudith Pierre-Victor is approved.

Dean Tomás R. Guilarte R.Stempel College of Public Health and Social Work

Andrés G. Gil

Vice President for Research and Economic Development and Dean of the University Graduate School

Florida International University, 2016 


\section{DEDICATION}

I acknowledge, first and foremost, the Creator of the universe, from whom all blessings flow! To Him I owe all that I am and all that I have. I acknowledge my Lord and Savior Jesus-Christ for His unconditional love and acceptance. I thank Him for His faithfulness. He has given me the courage to pursue my dreams despite my numerous health challenges.

I dedicate this work to my aunt, Félicité Pierre, and my uncles, Wilson Émilson Pierre and Frandieu Pierre-Victor. I wish my uncles were here to celebrate this accomplishment with me. To my parents, Helene Gesurin and Fabius Pierre, I dedicate this work. They have always offered their unwavering support. I thank all my siblings

for their support in this endeavor. To my cousin, Rosette Pierre Étienne and her family, I owe sincere gratitude. I am grateful to all my family members for their support.

I thank Saingel Benoit, Jécolia and John Pascal for their exemplifying perseverance to me. I give thanks to Newsoul Lapaix for the emotional support she has provided when it was most needed. To Martha and Paul Mathias I am forever indebted for their contribution to not only my intellectual but also my spiritual growth. I am grateful to the Social and Health Disparities Research Lab (SAHDRL) for providing the emotional support I needed to cross the finish line. I am indebted to Dr. Mary ShawRidley for her career development mentoring. She has provided tremendous guidance that helped me prepare for a postdoctoral opportunity. 


\section{ACKNOWLEDGMENTS}

I am truly grateful to my dissertation committee and chair for their guidance in this long journey. I am indebted to Dr. Trepka for being so generous with her time, for helping think through the research problems I wanted to address. I thank Dr. Page for the long hours he spent explaining the policy analysis process and many other concepts. Dr. $\mathrm{Li}$ has done the same for me in statistics. For that, I am grateful to him. I thank Dr. Stephens for her guidance not only in the dissertation but also in the qualitative research project that I have conducted. I am indebted to Dr. Madhivanan for her support and guidance in this process. I am appreciative of their patience and guidance.

I thank all the professors in the Epidemiology department for their contribution to my intellectual growth. To all my teachers from kindergarten to Lycée Marie-Jeanne in Haiti, I owe sincere gratitude.

I am grateful to Dr. Ahmed for encouraging me to apply for the McKnight Doctoral Fellowship through which my graduate studies have been supported. I am also grateful to Dr. Sonja Montas-Hunter. I am forever indebted to the Florida Education Fund staff for the emotional and financial support that were invaluable in the journey.

This research was supported by the Florida Education Fund through the McKnight Doctoral Fellowship (2014-2015) and by the Florida International University Graduate School Dissertation Year Fellowship (2015-2016). 


\section{ABSTRACT OF THE DISSERTATION \\ HUMAN PAPILLOMAVIRUS INFECTION AND VACCINATION POLICIES IN THE AMERICAN SOUTH}

by

Dudith Pierre-Victor

Florida International University, 2016

Miami, Florida

\section{Professor Purnima Madhivanan, Major Professor}

In the United States, the South has a disproportionate burden of cervical cancer, yet research reporting regional prevalence of human papillomavirus (HPV) infection is scarce. Since 2008, Virginia has passed a HPV vaccine mandate and Louisiana a HPV education bill. This dissertation estimated the prevalence of HPV infection among females and assessed the impact of Virginia's and Louisiana's HPV vaccination policy on vaccination among adolescent females.

The first manuscript estimated the prevalence of HPV infection using data from 4,250 females collected during the 2007-2010 National Health and Nutrition Examination Survey. Among 14-26 year-olds, the prevalence of high-risk oncogenic HPV was $25.6 \%$ (95\% CI: 22.4-33.3) in the South and 29.1\% (95\% CI: 24.8-33.8) in the rest of the country $(p=0.15)$. Among $27-59$ year-olds, infection rates were $20.9 \%(95 \%$ CI: 17.4-24.9) for the South and $14.5 \%$ (95\% CI: 12.9-16.3) for the rest of the country $(\mathrm{p}=0.0001)$ 
The second manuscript assessed the impact of Virginia's HPV vaccine mandate on vaccination using National Immunization Survey-Teen 2008-2012 data $(\mathrm{n}=3,203)$. A difference-in-differences estimation and logistic regression analysis were performed with South Carolina and Tennessee serving as comparison states. Virginia's mandate was not associated with an increase in vaccination rates. Physician recommendation was strongly associated with vaccination in the Virginia-South Carolina $(\mathrm{aOR}=10.3 ; \mathrm{p}=0.0001)$ and Virginia-Tennessee analyses (aOR=9.33; 95\%CI: 6.11-14.3).

The third manuscript assessed the impact of Louisiana's HPV education policy on vaccination using difference-in-differences estimation and logistic regression analysis, with Alabama and Mississippi as comparison states $(n=2,327)$. There was no evidence that the policy increased vaccination rates. Physician recommendation was associated with vaccination in the Louisiana-Alabama $(\mathrm{aOR}=7.74 ; 95 \% \mathrm{CI}$ : 5.22-11.5) and Louisiana-Mississippi comparison $(\mathrm{aOR}=7.05 ; 95 \% \mathrm{CI}$ : 4.6-10.5).

This study found a higher prevalence of HPV infection among females aged 2759 years in the South compared to the rest of the country. Additionally, physician recommendation was strongly associated with vaccination despite HPV policy implementation. These findings highlight the importance of physician recommendation for HPV vaccination and the need for recommended cervical cancer screening, particularly in the South. 


\section{TABLE OF CONTENTS}

CHAPTER

PAGE

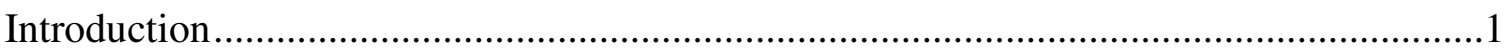

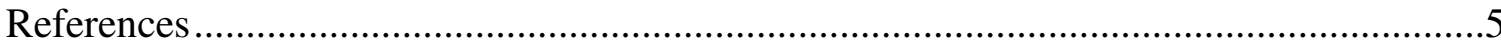

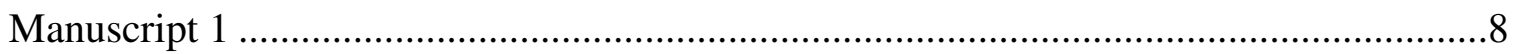

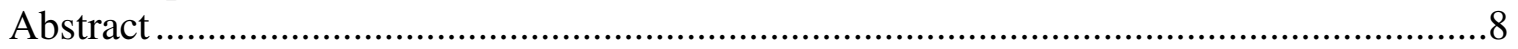

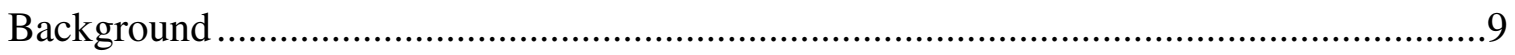

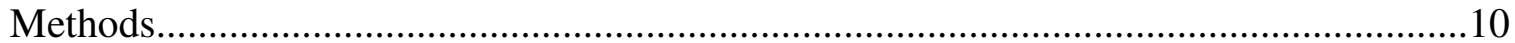

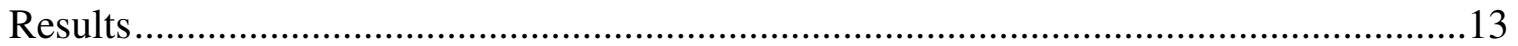

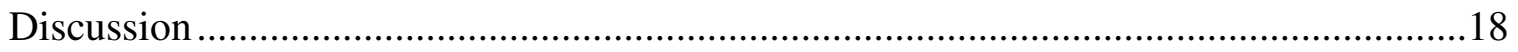

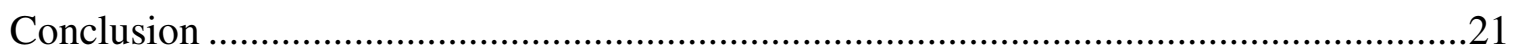

References

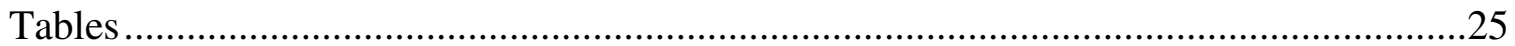

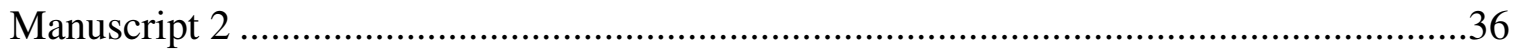

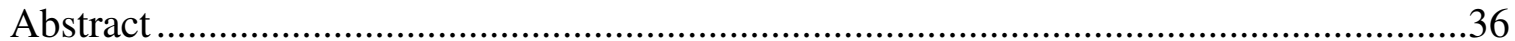

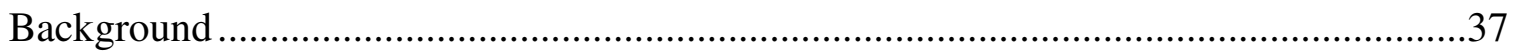

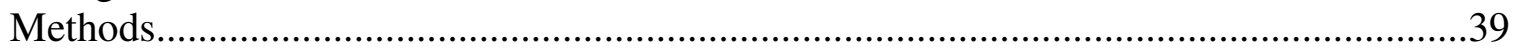

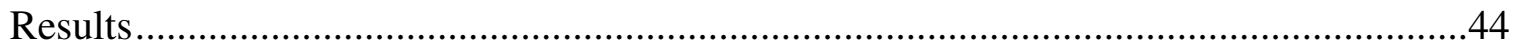

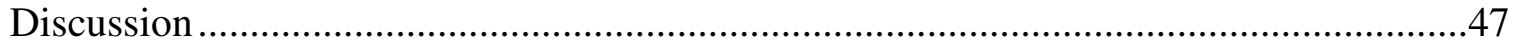

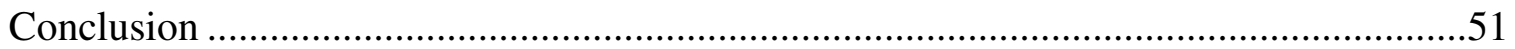

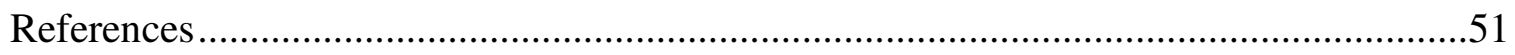

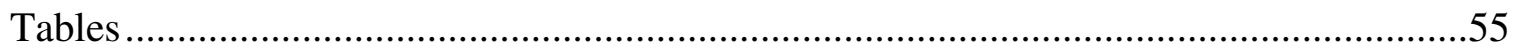

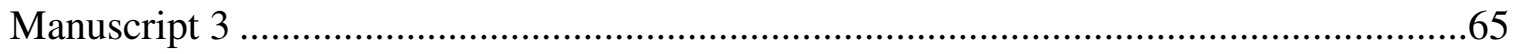

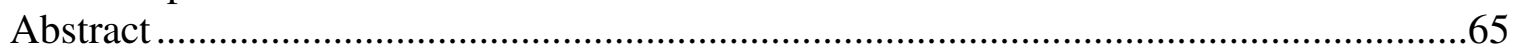

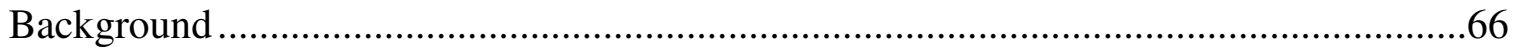

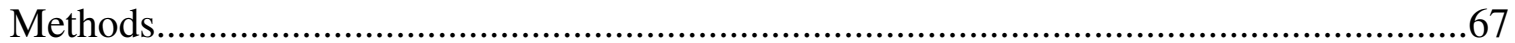

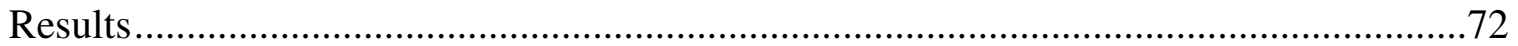

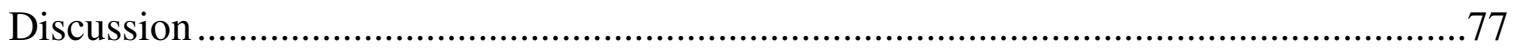

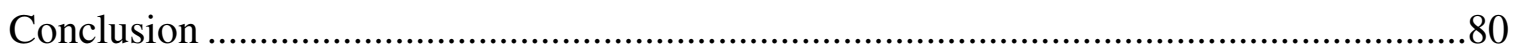

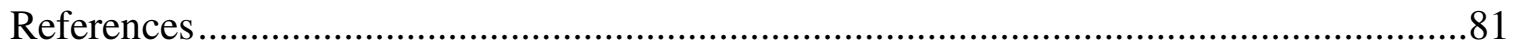

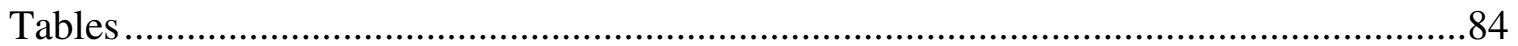

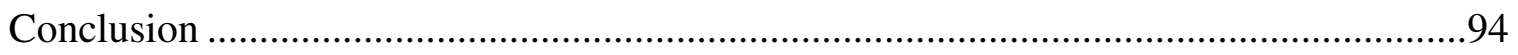

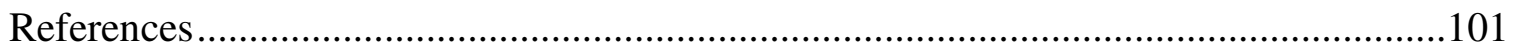

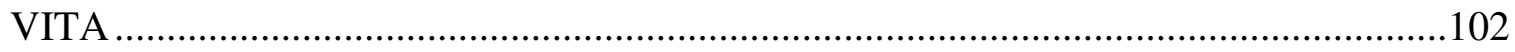




\section{LIST OF TABLES}

TABLES

PAGE

Manuscript 1

1. Prevalence of high-risk oncogenic, oncogenic and non-oncogenic types HPV

2. Socio-demographic and behavioral characteristics associated with infection

from high risk types among 14-26 year-old females .26

3. Socio-demographic and behavioral characteristics associated with infection from any oncogenic types among 14-26 year-old females

4. Socio-demographic and behavioral characteristics associated with infection from high-risk oncogenic types among 27-59 year-old females

5. Socio-demographic and behavioral characteristics associated with infection from any oncogenic types among 27-59 year-old females

6. Odds of HPV infections among sexually active 14-26 year-old females by sociodemographic and sexual behavioral characteristics

7. Odds of HPV infections among sexually active 27-59 year-old females by sociodemographic and sexual behavioral characteristics

Manuscript 2

1. Difference-in-differences in HPV vaccination rates, Virginia and South Carolina, and Virginia and Tennessee

2. Difference in selected demographic characteristics in Virginia and South

Carolina, and Virginia and Tennessee, pre-policy period.

3. Difference in selected demographic characteristics in Virginia and South

Carolina, and Virginia and Tennessee, post-policy period....

4. Odds ratios for HPV vaccination according to selected characteristics,

Virginia and South Carolina, and Virginia and Tennessee

5. Difference-in-differences in HPV vaccine Recommendation rates, Virginia

and South Carolina, and Virginia and Tennessee

6. Odds ratios for HPV vaccine recommendation according to selected characteristics, Virginia, South Carolina, and Tennessee 


\section{Manuscript 3}

1. Difference-in-differences in HPV vaccination rates, in Louisiana and Alabama, Louisiana and Mississippi

2. Difference in selected demographic characteristics in Louisiana and Alabama, Louisiana and Mississippi, pre-policy period.

3. Difference in selected demographic characteristics in Louisiana and Alabama, Louisiana and Mississippi, post-policy period

4. Odds ratios for HPV vaccination according to selected characteristics

Louisiana and Alabama, Louisiana and Mississippi

5. Difference-in-differences in HPV vaccine Recommendation rates, Louisiana and Alabama, Louisiana and Mississippi

6. Odds ratios for HPV vaccine recommendation according to selected characteristics, Louisiana and Alabama, Louisiana and Mississippi 


\section{ABBREVIATIONS AND ACRONYMS}

ACASI

$\mathrm{aOR}$

CAPI

CI

DiD

HPV

MEC

NHANES

NIS-Teen

SB

STI

Svy
Audio computer-assisted personal self-interview

Adjusted odds ratio

Computer-assisted personal interview

Confidence interval

Difference-in-differences

Human papillomavirus

Mobile Examination Center

National Health and Nutrition Examination Survey

National Immunization Survey-Teen

Senate Bill

Sexually transmitted infection SB

Survey 


\section{Introduction}

The southern region has long been known for its distinctiveness from the rest of the country, as evidenced in a letter written by Thomas Jefferson in 1785 (Savitt \& Young, 1988). Several factors, including its unique Protestant Evangelical Fundamentalism, contributed to that distinctiveness. One of the most salient characteristics of the South is disease (Savitt \& Young, 1988). Malaria, yellow fever, typhoid fever, hookworm and pellagra were prevalent in the South compared to other United States regions (Savitt \& Young, 1988). In the Colonial years, these diseases took a heavy toll on both poor white southerners as well as the large slave population on the plantations. After the civil war, the South's reputation for the poorest health in the nation suppressed immigration and investment thereby further delaying social and economic development (Savitt \& Young, 1988). In the $21^{\text {st }}$ century, the South continues to maintain its distinctiveness. The proportion of individuals of Black/African descent in the region since the time of slavery, rurality, poor socioeconomic living conditions, and health disparities (Wimberley \& Morris, 1996; Wimberly \& Morris, 1997) being among the principal factors contributing to its distinctiveness.

The South is comprised of 17 states: Delaware, District of Columbia, Florida, Georgia, Maryland, North Carolina, South Carolina, Virginia, West Virginia, Alabama, Kentucky, Mississippi Tennessee, Arkansas, Louisiana, Oklahoma and Texas (U.S. Census Bureau, 2013b). A region of the South has also been identified as the Black Belt, which spans across 11 states (Wimberley \& Morris, 1996; Wimberly \& Morris, 1997). This particular region is comprised of counties with the percentage of African Americans 
ranging from $18.5 \%$ to $26.7 \%$ (Wimberley \& Morris, 1996; Wimberly \& Morris, 1997).

The Southern Black Belt is where Southern rurality, poor socio-economic conditions, and health disparities meet. When the distribution of most chronic diseases-such as cardiovascular diseases and diabetes - are mapped, the South or Sotheastern belt stands out, indicating higher prevalence than the rest of the country (Barker, Kirtland, Gregg, Geiss, \& Thompson, 2011; Devesa et al., 1999; Lanska \& Kuller, 1995).

Cervical cancer is among the chronic conditions that disparately plague the South (Devesa et al., 1999; Howlader et al., 2013; U.S. Cancer Statistics Working Group, 2015). In 2010, the top ten states with the highest incidence and mortality rates for cervical cancer were all located in the South (Howlader et al., 2013). In 2012, the incidence of cervical cancer in the United States was 7.4 per 100,000 (U.S. Cancer Statistics Working Group, 2015). Only Maryland, Virginia, North and South Carolina had incidence rates below the national average. A similar geographic distribution is reported for cervical cancer mortality (U.S. Cancer Statistics Working Group, 2015).

Human papillomavirus (HPV), the leading sexually transmitted infection (Cates, 1999; Weinstock, Berman, \& Cates, 2004), has been consistently linked to oropharyngeal and anogenital cancers (Bosch, Lorincz, Muñoz, Meijer, \& Shah, 2002; Endo, Yamashita, Jin, Akutsu, \& Jimbow, 2003; Gissmann \& zur Hausen, 1980; Jones, Rowan, \& Stewart, 2005). The Food and Drug Administration had licensed the quadrivalent HPV vaccine (HPV4, Gardasil) in 2006, which protects against HPV 6,11,16, and 18 (Centers for Disease Control and Prevention, 2010b); the bivalent HPV vaccine (HPV2, Cervarix) in 2009, which confers protection against HPV 16 and 18 (Centers for Disease Control and 
Prevention, 2010a); and the nanovalent HPV vaccine (Gardasi19) in 2014, which confers protection against HPV 6,11,16,18,31,33,45,52, and 58 (U.S. Food and Drug Administration, 2014). The HPV vaccine has a three-dose schedule recommended for boys and girls 11-12 years of age and catch-up vaccination for 13-26 year-olds (Centers for Disease Control and Prevention, 2013a). Currently, HPV vaccines are administered in primary health care settings (Herzog, Huh, \& Einstein, 2010) including school-based health care centers (Lofink et al., 2013).

Since the licensure of the vaccine, individual states have been enacting HPV vaccination policies to address funding for HPV education or HPV vaccination (National Conference of State Legislatures, 2015). Several states have passed bills requiring HPVrelated education and HPV vaccine awareness for adolescents, parents, or both. Indiana, Utah, Iowa, New Jersey, and Washington took the lead on HPV and HPV vaccine awareness legislatures in the 2006-2007 period (National Conference of States Legislatures, 2015). Among Southern states, only the District of Columbia and Virginia had passed a HPV vaccine mandate for school-entry while North Carolina and Louisiana required that schools provide HPV vaccine education to parents of preteens and teens in specific grades (National Conference of State Legislatures, 2015). Virginia's HPV vaccine mandate requires girls entering sixth grade to receive at least one dose of the HPV vaccine. Louisiana requires schools to provide HPV/HPV vaccine information to parents of adolescents in grades 6-12. Both policies were enacted in 2008 (National Conference of State Legislatures, 2015). 
While HPV infection rates are available at the national level, there is a paucity of research that examines geographic variation in HPV infection prevalence. Women residing in the American South are disproportionately affected by cervical cancer. However, research studies estimating HPV infection prevalence and those examining socio-demographics and sexual behaviors associated with HPV infection are scarce. Such information is crucial to increase cervical cancer prevention in order to reduce cancer disparities.

Despite the availability of HPV vaccine for the last nine years, vaccination rates are low among American adolescents (Centers for Diseases Control and Prevention, 2013). Vaccination rates are particularly low in the Southern region, notwithstanding its high cervical cancer rates. In 2012, among 13-17 year-old females HPV vaccination rates were lower in the South (48.9\%) compared to the Midwest (50.5\%), the Northeast (58.2\%), the West (61.4\%), and the national average (53.8\%) (Centers for Disease Control and Prevention, 2013b). Virginia's HPV vaccine mandate for school-entry and Louisiana's HPV vaccine awareness policies could serve as models for other Southern states if they are effective. While several states have introduced similar HPV vaccine awareness policies and HPV vaccine mandate for school-entry (National Conference of State Legislatures, 2015), these policies have not been evaluated.

In light of cervical cancer disparities in the Southern region, the impact evaluation of Virginia's HPV vaccine mandate as a school-entry requirement and Louisiana's HPV vaccine education policies requiring HPV and HPV vaccine education for parents and students is crucial. To contribute to this body of literature, the first manuscript estimated 
the prevalence of HPV infection in Southern region compared to the rest of the country.

Findings will shed light on the burden of HPV infection in the region in addition to highlighting HPV infection disparities. Such information can guide national cervical cancer prevention efforts to allocate limited resources to areas with greater needs. The second manuscript assessed the impact of Virginia's HPV vaccine mandate as a schoolentry requirement for girls in the sixth grade on HPV vaccination among 13-17 year-old

females. The third manuscript assessed the impact of Louisiana's law requiring HPV and HPV vaccine education for parents and students on HPV vaccination among 13-17 yearold females. HPV vaccine mandate for school-entry and HPV education legislature have been introduced in several states (National Conference of State Legislatures, 2015).

Consequently, the impact assessment of both policies will shed light on whether parental HPV awareness or HPV vaccine mandate for school-entry is effective to increase HPV vaccination. Together, the second and the third manuscripts will provide policy-makers with information that can assist them in deciding the best HPV vaccination policies.

\section{References}

Barker, L. E., Kirtland, K. A., Gregg, E. W., Geiss, L. S., \& Thompson, T. J. (2011). Geographic distribution of diagnosed diabetes in the U.S. American Journal of Preventive Medicine, 40(4), 434-439. doi:10.1016/j.amepre.2010.12.019

Bosch, F. X., Lorincz, A., Muñoz, N., Meijer, C. J. L. M., \& Shah, K. V. (2002). The causal relation between human papillomavirus and cervical cancer. Journal of Clinical Pathology, 55, 244-265.

Cates, W. J. (1999). Estimates of the incidence and prevalence of sexually transmitted diseases in the United States: American Social Health Association Panel. Sexually Transmitted Diseases, 26(4 suppl), S2-7.

Centers for Disease Control and Prevention. (2010a). FDA licensure of bivalent human papillomirus vaccine (HPV2, Cervarix) for use in females and updated HPV vaccination recommendations from the Advisory Committee on Immunization 
Practices (ACIP). MMWR, 59(20), 625-629.

Centers for Disease Control and Prevention. (2010b). FDA licensure of quadrivalent human papillomavirus vaccine (HPV4, Gardasil) for use in males and guidance from the Advisory Committee on Immunization Practices (ACIP). MMWR, 56(20), 630632.

Centers for Disease Control and Prevention. (2013a). Immunization schedules: Immunization schedules for preteens and teens in easy-to-read formats. Retrieved March 13, 2015, from http://www.cdc.gov/vaccines/schedules/easy-to-read/preteenteen.html

Centers for Disease Control and Prevention. (2013b). National and state vaccination coverage among adolescents aged 13-17 years- United States, 2012. MMWR, 62(34), 685-693.

Centers for Diseases Control and Prevention. (2013). National and state vaccination coverage among adolescents aged 13-17 years--United States, 2012. MMWR, 62(34), 685-93. Retrieved from http://www.ncbi.nlm.nih.gov/pubmed/23985496

Devesa, S. S., Grauman, D. J., Blot, W. J., Pennello, G. A., Hoover, R. N., \& Fraumeni, J. F. (1999). Atlas of Cancer Mortality in the United States, 1950-94. Bethesda, MD. Retrieved from http://ratecalc.cancer.gov/archivedatlas/pdfs/text.pdf

Endo, M., Yamashita, T., Jin, H.-Y., Akutsu, Y., \& Jimbow, K. (2003). Detection of human papillomavirus type 16 in bowenoid papulosis and nonbowenoid tissues. International Journal of Dermatology, 42, 474-6. Retrieved from http://www.ncbi.nlm.nih.gov/pubmed/12786880

Gissmann, L., \& zur Hausen, H. (1980). Partial characterization of viral DNA from human genital warts (Condylomata acuminata). International Journal of Cancer, 25, $605-609$.

Herzog, T. J., Huh, W. K., \& Einstein, M. H. (2010). How does public policy impact cervical screening and vaccination strategies? Gynecologic Oncology, 119(2), 17580. doi:10.1016/j.ygyno.2010.08.021

Howlader, N., Noone, A. M., Krapcho, M., Garshell, J., Neyman, N., Altekruse, S. F., ... Cronin, K. A. (2013). SEER Cancer Statistics Review, 1975-2010. Bethesda, MD. doi:based on November 2012 SEER data submission, posted to the SEER web site, April 2013

Jones, R. W., Rowan, D. M., \& Stewart, A. W. (2005). Vulvar intraepithelial neoplasia: Aspects of the natural history and outcome in 405 women. Obstetrics and Gynecology, 106(6), 1319-1326.

Lanska, S. J., \& Kuller, L. H. (1995). The geography of stroke in the United States and the concept of a stoke belt. Stroke, 26, 1145-49.

Lofink, H., Kuebler, J., Juszczak, L., Schlitt, J., Even, M., Rosenberg, J., \& White, I. 
(2013). 2010-2011 Census Report of of School-Based Health Centers. Washington, D.C. Retrieved from www.sbh4all.org

National Conference of State Legislatures. (2015). HPV vaccine: State legislation and statutes. Retrieved September 25, 2013, from http://www.ncsl.org/issuesresearch/health/hpv-vaccine-state-legislation-and-statutes.aspx

National Conference of States Legislatures. (2015). HPV Vaccine: State Legislation and Statutes. Retrieved December 24, 2015, from

http://www.ncsl.org/research/health/hpv-vaccine-state-legislation-and-statutes.aspx

Savitt, T. L., \& Young, J. H. (Eds.). (1988). Disease and distinctiveness in the American South (1st ed.). Knoxville, TN: The University of Tennessee Press.

U.S. Cancer Statistics Working Group. (2015). United States Cancer Statistics:19992012 cancer incidence and mortality Web-based report. Atlanta, Georgia. Retrieved from www.cdc.gov/uscs.

U.S. Census Bureau. (2013). Regions and divisions - 2007 Economic Census. Retrieved from http://www.census.gov/econ/census07/www/geography/regions_and_divisions.html

U.S. Food and Drug Administration. (2014). Press Announcements - FDA approves Gardasil 9 for prevention of certain cancers caused by five additional types of HPV. Retrieved June 18, 2015, from http://www.fda.gov/NewsEvents/Newsroom/PressAnnouncements/ucm426485.htm

Weinstock, H., Berman, S., \& Cates, W. J. (2004). Sexually transmitted diseases among American youth : Incidence and prevalence estimates, 2000. Perspectives on Sexual and Reproductive Health, 36(1), 6-10.

Wimberley, R. C., \& Morris, L. V. (1996). The reference book on regional well-being: U.S. regions, the Black Belt, Appalachia. Raleigh, NC: Southern Rural Development.

Wimberly, R. C., \& Morris, L. V. (1997). The Southern Black Belt: A national perspective. Lexington, KY: TVA Rural Studies Press. 


\title{
Manuscript 1
}

\section{Human Papillomavirus Infections in the American South and Other United States Regions}

\begin{abstract}
Human papillomavirus (HPV) infection is the most common sexually transmitted infection worldwide and has been linked to several cancers, including cervical cancer. In the United States, the Southern region has a disproportionate burden of cervical cancer, and research about the epidemiology of HPV in the region is scarce. This study estimates the prevalence and correlates of HPV infections among 14-59 year-old females.
\end{abstract}

Data from 4,250 females aged 14-59 years collected during the 2007-2010 National Health and Nutrition Examination Survey were used. The prevalence of HPV infection was estimated for the South, Northeast, Midwest, and West combined. Weighted chi-square test and logistic regression were performed to examine the association between HPV infection and socio- and behavioral demographics.

Among 14-26 year-old females, the prevalence of high-risk oncogenic HPV types was $25.6 \%$ (95\% confidence (CI): 22.4-33.3) in the South and 29.1\% (95\% CI: $24.8-$ 33.8) in the other regions $(\mathrm{p}=0.15)$. Among 27-59 year-old women, infection with highrisk oncogenic types was $20.9 \%$ (95\% CI: 17.4-24.9) in the South compared to $14.5 \%$ (95\% CI: 12.9-16.3) in other regions $(\mathrm{p}=0.0001)$.

This study found a higher prevalence of infection from high-risk oncogenic types among 27-59 year-olds. These findings indicate the importance of promoting HPV vaccination as well as cervical cancer screening, particularly in the Southern region of the United States. 


\section{Background}

Human papillomavirus (HPV) is the most common sexually transmitted infection (STI) worldwide and has been consistently linked to head, neck, pharyngeal, and anogenital cancers (Forman et al., 2012). Over 100 HPV types infect humans, with 40 of these HPV types infecting mainly the anogenital tract (Franco, Duarte-Franco, \& Ferenczy, 2001). Most HPV-related cancers result from infections from HPV types 16 and 18 (Muñoz et al., 2003). According to their association with pre-malignancy and invasive cancer, HPV types are classified as high- or low-risk oncogenic, and nononcogenic (Bosch et al., 2002; Franco et al., 2001; Wright, Denny, \& Kuhn, 2000).

Cervical cancer is the fourth most common cancer worldwide and disproportionately affects women in developing and underdeveloped countries. Among HPV-related cancers, cervical cancer is the most prevalent. In developed countries, cervical cancer affects poor and disadvantaged women (International Agency for Research on Cancer, 2014). In the United States, cervical cancer mainly affects disadvantaged, poor women with limited access to healthcare such as those living in the Appalachian (Devesa et al., 1999; Horner et al., 2011), and the Southern regions (Devesa et al., 1999). In 2010, the national cervical cancer incidence was 7.6 per 100,000 . The seven states with the highest cervical cancer incidence rates were all located in the South: West Virginia (11.6 per 100,000), Arkansas (10.7), Oklahoma (10.3), Mississippi (10.2), Washington D.C. (9.7), Texas (9.4), and Louisiana (9.1) (Howlader et al., 2013).

Women residing in the American South are disproportionately affected by cervical cancer. However, research studies estimating prevalence of HPV infection and 
associated socio-demographic characteristics and sexual behaviors are lacking in the region. Such information is crucial to increase HPV vaccination and cervical cancer screening in order to reduce cancer disparities. The present study estimates the prevalence of HPV infection from high-risk oncogenic, any oncogenic, and nononcogenic types and examines the correlates of HPV infections in the American South. We hypothesized that the rates of infection from high-risk HPV types would be higher in the South than in the rest of the United States.

\section{Methods}

\section{Study Design and Population}

The National Health and Nutrition Examination Survey (NHANES) data are collected through a multifaceted probability sampling strategy in order to obtain a representative sample of the noninstitutionalized population in the United States (Curtin, Lester et al., 2013). NHANES detailed methodologies have been published elsewhere (Curtin, Lester et al., 2013; Zipf et al., 2013). NHANES data collection occurs in two stages: a home interview and a health examination. Upon selection, participants are first screened to ensure eligibility. Eligible participants complete the home interview and are invited to the Mobile Exam Center (MEC) for computer-assisted personal interview (CAPI) questionnaires, audio computer-assisted personal self-interview (ACASI) questionnaires, examinations, and biological specimen collection (Zipf et al., 2013).

From 2003 to 2010, females 14-59 years are asked to self-collect vaginal samples. Several studies have found self-collected vaginal swabs to be slightly less or as sensitive as physician-collected samples (Bhatla et al., 2009; Ogoina, Musa, \& 
Onyemelukwe, 2013; Petignat et al., 2007; Zhao et al., 2012). A systematic review comparing self-collected and physician-collected samples for low- and high-risk types HPV DNA detection from 18 studies estimated the average detection rate at $27.4 \%$ (26.228.6) for self-sampling and 28.0\% (26.8-29.1) for physician-sampling (Petignat et al., 2007). NHANES reports HPV test results as positive, negative, inadequate or missing for 40 HPV types.

Two NHANES survey cycles, 2007-2008 and 2009-2010, were combined to maximize the sample for the analysis. A total of 4,250 females aged 14-59 years provided adequate self-collected vaginal samples for HPV DNA detection from 2007 to 2010. Based on HPV vaccine eligibility, the sample was divided into those still eligible for HPV vaccine (14-26 years) and those no longer eligible for HPV vaccine (27-59 years).

\section{Variables}

HPV infection status was the outcome of interest. Following the classification scheme developed by several researchers (Bosch et al., 2002; Bouvard et al., 2009; Franco et al., 2001), HPV types 16, 18, 31, 33, 35, 39, 45, 51, 52, 56, 58, and 59 were classified as high-risk oncogenic types. HPV types $6,11,16,18,31,33,35,39,40,42$, $45,51,52,53,55,56,58$, and 59 were classified as oncogenic types. The remaining HPV types were classified as non-oncogenic.

Socio-demographic variables from the home interview survey, sexual behavior variables from the ACASI questionnaires, reproductive health variables from the CAPI questionnaires, and HPV infection status from the laboratory data were merged for the 
analysis. HPV infection status, race/ethnicity, country of birth, country of citizenship, federal poverty level, health insurance status, healthcare utilization variables, HPV infection status, use of contraception, duration of contraception use, age at first sexual intercourse, and number of lifetime partners were included in the analysis. Females who tested positive for at least one of the high-risk oncogenic types were classified as being infected from high-risk oncogenic HPV types. The same criterion was used for any oncogenic types. Since the region and state of residence are restricted variables, the dataset was accessed and analyzed at the Restricted Data Center (Atlanta, GA). Furthermore, to prevent potential disclosure, the region rather than the state of residence of survey respondents was specified in the dataset.

\section{Statistical Analysis}

STATA svy (StataCorp, 2013) commands were used to conduct the analyses to account for the complex multistage study design and sample weight. Since two survey cycles were combined, NHANES guidelines were followed to compute the new MEC sample weight used in the analysis. Women 14-59 years of age from all racial/ethnic groups were included in the analysis. The proportion of respondents who tested positive for high-risk oncogenic, any oncogenic, and non-oncogenic HPV types for 2007-2010 were computed for the South separately and the three other regions combined (Northeast, Midwest, and West). A 95\% confidence interval was computed for each proportion. Weighted chi-square analyses were performed to examine the association between HPV infection and important demographic, healthcare utilization, and sexual behavior variables. Variables that were significantly associated with HPV infection from high-risk 
oncogenic and any oncogenic type HPV and sexual behavior variables were included in a multivariable logistic regression to identify factors independently associated with HPV infection among sexually active women. We performed analysis with all females for whom the variables of interest were present. A 5\% significance level was used for all analyses.

\section{Results}

\section{HPV Prevalence}

\section{4-26 year-old females}

The prevalence of high-risk oncogenic types was $25.6 \%$ (95\% CI: $22.4-33.3$ ) in the South and $29.1 \%$ (95\% CI: 24.8-33.8) in the rest of the U.S. (p=0.15). The prevalence of any oncogenic type was 31.7\% (95\% CI: 26.4-37.6) in the South and $32.3 \%$ (95\% CI: 27.9-36.9) in other regions $(\mathrm{p}=0.84)$. Non-oncogenic type prevalence was $36.5 \%$ (95\% CI: 30.0-43.4) in the South and 31.9\% (95\% CI: 26.9-37.4) in the other regions $(\mathrm{p}=0.08)($ Table 1$)$.

27-59 year-old women

Infection with high-risk oncogenic types was $20.9 \%$ (95\% CI: 17.4-24.9) in the South compared to $14.5 \%$ (95\% CI: $12.9-16.3)$ in other regions $(\mathrm{p}=0.0001)$. For infection from any oncogenic types, infection rates were $24.0 \%$ (95\% CI: 19.9-28.7) and $17.9 \%$ (95\% CI: 16.3-19.5) for the South and other regions respectively $(\mathrm{p}=0.0001)$ (Table 1).

[Table 1 Here] 


\section{Demographics Associated with HPV Infection}

\section{4-26 year-old females}

In the South, infection from high-risk oncogenic type HPV varied significantly by race/ethnicity, history of contraceptive use, sexual activity status, and number of lifetime sex partners (Table 2). Among women living in the rest of the country, infection with high-risk oncogenic types differed by marital status, poverty index, insurance status, history of contraceptive use, sexual activity status, number of lifetime sex partners, and having had at least one sex partner five years older (Table 2). In the South, infection from any oncogenic types varied by race/ethnicity, history of contraceptive use, sexual activity status, and number of lifetime sex partners (Table 3). In the rest of the country, infection from any oncogenic types varied by marital status, insurance status, history of contraceptive use, sexual activity status, and number of lifetime sex partners (Table 3 ).

[Table 2 Here]

[Table 3 Here]

\section{7-59 year-old women}

In the Southern region, infection from high-risk oncogenic HPV types varied significantly by race/ethnicity, country of birth, marital status, poverty index, insurance status, and number of lifetime sex partners (Table 4). Among women living in other US regions, infection from high-risk oncogenic types differed by race/ethnicity, marital status, poverty index, insurance status, type of place one receives care, healthcare utilization, age at first sexual intercourse, number of sex partners 5 years or older, and 
number of lifetime sex partners (Table 4). For the Southern region, infection from any oncogenic types varied by race/ethnicity, country of birth, marital status, poverty index, insurance status, and number of lifetime sex partners (Table 5). In the rest of the country, infection from any oncogenic types differed by race/ethnicity, marital status, poverty index, type of place respondents receive care, age at first sexual intercourse, number of lifetime sex partners, and having had at least one sex partner five years older (Table 5).

[Table 4 Here]

[Table 5 Here]

\section{Correlates of HPV Infection among Sexually Active Women}

\section{4-26 year-old females}

Among 14-26 year-old females in the South, those whose income was 300-499\% above the federal poverty level had lower odds of infection from high-risk HPV oncogenic types compared to those whose income was below the federal poverty level (adjusted odds ratio $(\mathrm{aOR})=0.36 ; \mathrm{p}<0.01)$. Additionally, females who had four or more lifetime sex partners had greater odds of infection with high-risk oncogenic types compared to those who had three or fewer sex partners $(\mathrm{aOR}=8.27 ; \mathrm{p}<0.001)$ (Table 6). Among 14-26 year-old females living in other regions, those who have never been married had greater odds to be infected from high-risk oncogenic types compared to those who were married $(\mathrm{aOR}=12.79 ; \mathrm{p}<0.001)$, and those who were divorced or separated had higher odds of infection $(\mathrm{aOR}=6.09 ; \mathrm{p}<0.001)$ (Table 6). 
For infection with any oncogenic types in the South, females whose income was 200-499\% above the poverty index had lower odds of infection compared to those whose income was below the poverty index $(\mathrm{aOR}=0.45 ; \mathrm{p}<0.05)$ (Table 6). Southern females who had insurance coverage had higher odds of infection compared to those who had no coverage $(\mathrm{aOR}=2.48 ; \mathrm{p}<0.01)$. Those who had four or more sex partners had higher odds of infection compared to those who had three or fewer partners $(\mathrm{aOR}=8.51 ; \mathrm{p}<0.001)$. Among females in other regions, those who had never been married had greater odds to be infected with high-risk oncogenic types compared to those who were married (aOR= $14.1 ; \mathrm{p}<0.001)$, and those who were divorced or separated had higher odds of infection $(\mathrm{aOR}=6.13 ; \mathrm{p}<0.001)$. Additionally, females who had four or more sex partners had higher odds of infection compared to those who had three or fewer sex partners $(\mathrm{aOR}=4.40 ; \mathrm{p}<0.001)($ Table 6$)$.

\section{[Table 6 Here]}

\section{7-59 year-old females}

Among 27-59 year-old women in the South, those who had never been married had increased odds to be infected with high-risk oncogenic types $(\mathrm{aOR}=5.14 ; \mathrm{p}<0.01)$, and the odds were also higher for those who were no longer married $(\mathrm{aOR}=2.98 ; \mathrm{p}<0.05)$ compared to married women (Table 7). In the other regions, women who had never been married $(\mathrm{aOR}=2.38 ; \mathrm{p}<0.01)$ and those who were previously married $(\mathrm{aOR}=4.26$; $\mathrm{p}<0.05)$ had higher odds of infection from high-risk oncogenic types. Women with household income $300-499 \%$ above the poverty index had lower odds to be infected $(\mathrm{aOR}=0.26 ; \mathrm{p}<0.01)$. Additionally, women who had one or more sex partners at least 
five years older $(\mathrm{aOR}=1.82 ; \mathrm{p}<0.01)$ and who had four or more sex partners $(\mathrm{aOR}=3.78$; $\mathrm{p}<0.01$ ) had greater odds to be infected (Table 7).

For any oncogenic types, in the South, women who had never been married had higher odds of infection $(\mathrm{aOR}=4.27 ; \mathrm{p}<0.01)$. In the rest of the country, women who had never been married had greater odds to be infected $(\mathrm{aOR}=2.20 ; \mathrm{p}<0.01)$ and those who were divorced, separated, or widowed also had increased odds to be infected $(\mathrm{aOR}=4.16$; p $<0.01$ ). Women whose income was 300-499\% above the poverty index had lower odds of infection from any oncogenic types $(\mathrm{aOR}=0.46 ; \mathrm{p}<0.01)$. Those who had four or more sex partners had higher odds of infection $(\mathrm{aOR}=3.90 ; \mathrm{p}<0.001)$, and those who had their sexual debut at 15 years or older had lower odds to be infected from any oncogenic types compared those who had their sexual debut at 14 years or younger $(\mathrm{aOR}=0.47 ; \mathrm{p}<0.01)$ (Table 7).

[Table 7 Here]

\section{Discussion}

Cervical cancer is more prevalent in the Southern region compared to the rest of the United States, and this study found that the prevalence of HPV infections from highrisk oncogenic types was higher in the South than the rest of the United States, among women aged 27-59 years but not among those 14-26 years old. Among 14-26 year-old females, having four or more lifetime sex partners was positively associated with infection from high-risk and any oncogenic types infection in the South and the rest of the country. Among 27-59 year-old females, having four or more lifetime sex partners was positively associated with infection from high-risk and any oncogenic types infection 
in the rest of the country but not in the South. Moreover, sexually active women who have never been married and those who were divorced, separated, or widowed to have a greater odds of infection from any oncogenic and high-risk oncogenic types.

The prevalence of infection from high-risk oncogenic types was higher in the South compared to the rest of the U.S. among 27-59 year-old females. Although there is limited literature investigating geographic variation in HPV infection, a study estimated HPV prevalence among women in the Appalachia, a region with high rates of cervical cancer and predominantly non-Hispanic White population (Reiter, Katz, Ruffin, et al., 2013). The study found higher prevalence of high-risk infection among Appalachian women (Reiter, Katz, Ruffin, et al., 2013) when compared to the national estimates for non-Hispanic White females (Hariri et al., 2011). The majority of the Appalachian women were between the ages of 18 and 40 years (Reiter, Katz, Ruffin, et al., 2013). The difference in the prevalence of infection from high-risk oncogenic types in the South compared to the rest of the U.S. among 27-59 year-old females appears to be smaller than the difference in cervical cancer incidence and mortality. Therefore, this difference in the prevalence is not large enough to account for the higher prevalence of cervical cancer in the South.

Among 14-26 year-olds, the prevalence of infection from high-risk oncogenic or any oncogenic types HPV was not higher in the South compared to the rest of the country. Using the 2003-2006 and the 2007-2010 NHANES survey cycles, a study investigated the change in the prevalence of infection from HPV types 6, 11, 16, or 18 (Markowitz et al., 2013). Among females aged 14-19 years, HPV infection prevalence 
declined from $11.5 \%$ in the $2003-2006$ cycle to $5.1 \%$ in the $2007-2010$ cycle. Similar decline was not observed in the older age-groups (Markowitz et al., 2013). Comparable rates of infection from high-risk or any oncogenic types between the South and the rest of the country among 14-26 year-olds are probably due to the national decline in HPV infection in the vaccine-eligible age-group.

Among 14-26 year-old females, this study found that having four or more lifetime sex partners was positively associated with infection from high-risk and any oncogenic types in the South and the rest of the country. However, among 27-59 yearold females, having four or more lifetime sex partners was positively associated with infection from high-risk and any oncogenic types in the rest of the country but not in the South. Previous research has reported that increasing number of lifetime sex partners was associated with HPV infection (Dunne et al., 2007; Markowitz et al., 2009; Reiter, Katz, Ruffin, et al., 2013). It is not clear as to why increasing number of sex partners was not associated with infection from high-risk or oncogenic types in the South.

The present study also found sexually active women who had never been married and those who were divorced, separated, or widowed had greater odds of infection from any oncogenic and high-risk oncogenic types. Several studies have reported higher prevalence of HPV infection among unmarried women (Dunne et al., 2007; Kahn et al., 2007; Reiter, Katz, Ruffin, et al., 2013). The absence of a long-term and committed sex partner facilitates multiple sexual partnerships or short-term sexual partnerships, which increase the risk of HPV infection. This may help explain the higher prevalence of 
infection from high-risk and any oncogenic types observed among unmarried sexually active women.

The results indicated that the prevalence of infection from high-risk oncogenic types was higher in the South compared to the rest of the U.S. among 27-59 year-old females. Public health efforts to increase cervical cancer screening in the South as well as efforts to increase HPV vaccination among vaccine-eligible females must continue in order to decrease the disparities in cervical cancer mortality. Healthy People 2020's goal is to increase the proportion of 21-65 year-old women who receive a cervical cancer screening based on the most recent guidelines to $93 \%$ (U.S. Department of Health and Human Services, 2014). In 2013, 78.5\% of women aged 21-65 years had a pap smear test in the past three years (National Institute of Health, 2015). This report found lower cervical cancer screening rates among women living $200 \%$ or below the poverty index and among women who had less than high school education (National Institute of Health, 2015). Doescher and colleagues reported that women with low socio-economic status, particularly those residing in rural areas, were less likely to be screened for cervical cancer (Doescher \& Jackson, 2009). Thus, Southern women of low socio-economic status, especially those living in rural areas, should be targeted for cervical cancer screening.

The present study had several limitations. First, sexual behaviors were selfreported, which inevitably lends to poor recall and social desirability bias. Sexual behavior is generally considered a private and sensitive matter. As a result, most individuals are not enthusiastic about revealing their sexual practices due to social 
stigma, embarrassment, or loss of confidentiality (Brener, Billy, \& Grady, 2003; Kelly, Soler-Hampejsek, Mensch, \& Hewett, 2013; O’Sullivan, 2008). Consequently, such behaviors may be underrepresented. In the same vein, previous research has reported that responses to ACASI collecting sexual behavior data are generally more accurate compared to face-to-face interviews (Ghanem, Hutton, Zenilman, Zimba, \& Erbelding, 2005; Phillips, Gomez, Boily, \& Garnett, 2010). NHANES collects sexual behavior data using ACASI thereby reducing social desirability bias in this study. Moreover, the vaginal swabs were self-collected, and HPV testing could not be performed for some respondents due to inadequate specimen collection. However, this study also had several strengths. First, the study used a nationally representative sample which is robust against selection bias. Additionally, NHANES accounts for participant non-response. As a result, these aspects lends to more reliable and valid findings. Moreover, the present study is among the few to have attempted to assess the prevalence of infections from high and low-risk HPV among 14-59 year-old women in a region with a disparate cervical cancer burden.

\section{Conclusion}

This study estimated the prevalence of infection among females for the South and the rest of the country and found a higher prevalence of infection from high-risk oncogenic and any oncogenic HPV types among 27-59 year-old Southern females compared to the rest of the country, but not among the 14-26 year-olds. Women in the Southern region remain at higher risk of developing and dying from cervical cancer, and the higher prevalence of infection from high-risk oncogenic and any oncogenic HPV 
types among 27-59 year-olds in the South partially explains the higher prevalence of cervical cancer in the South. However, the difference in HPV prevalence in the older age-group seems to be smaller than the difference in cancer incidence between the South and other regions. This suggests that the disparities in cervical cancer incidence are not only being driven by the differences in the epidemiology of HPV alone, but also by disparities in cervical cancer screening. Efforts to make cervical cancer screening accessible to disadvantaged women in the region must continue in order to reduce disparities in cervical cancer mortality.

Among 14-26 year-olds, the prevalence of infection from high-risk oncogenic and any oncogenic types HPV was relatively high in the South and the rest of the country. These findings reiterate the need for HPV vaccination to be administered to preteens, prior to their sexual debut. Additionally, females aged 14-26 years are still eligible for HPV vaccination catch-up. Consequently, healthcare providers should recommend the vaccine to 14-26 year-old females even if they are already infected with one HPV type as they can be protected from other HPV types covered by the vaccine.

\section{References}

Bhatla, N., Dar, L., Patro, A. R., Kumar, P., Kriplani, A., Gulati, A., ... Gravitt, P. E. (2009). Can human papillomavirus DNA testing of self-collected vaginal samples compare with physician-collected cervical samples and cytology for cervical cancer screening in developing countries ? Cancer Epidemiology, 33, 446-450. doi:10.1016/j.canep.2009.10.013

Bosch, F. X., Lorincz, A., Muñoz, N., Meijer, C. J. L. M., \& Shah, K. V. (2002). The causal relation between human papillomavirus and cervical cancer. Journal of Clinical Pathology, 55, 244-265.

Bouvard, V., Baan, R., Secretan, B., El Ghissassi, F., Benbrahim-Tallaa, L., Guha, N., ... 
Cancer Monograph Working Group. (2009). Special Report : A review of human carcinogens — Part B : biological agents. Lancet Oncology, 10(4), 321-322. doi:10.1016/S1470-2045(09)70096-8

Brener, N. D., Billy, J. O. G., \& Grady, W. R. (2003). Assessment of factors affecting the validity of self-reported health-risk behavior among adolescents: Evidence from the scientific literature. Journal of Adolescent Health, 33(6), 436-457. doi:10.1016/S1054-139X(03)00052-1

Curtin, Lester, R., Mohadjer, L. K., Dohrmann, S. M., Kruszan-Moran, D., Mirel, L. B., Carroll, M. D., ... Johnson, C. L. (2013). National Health and Nutrition Examination Survey: Sample design, 2007 - 2010. Vital and Health Statistics, 2(160).

Devesa, S. S., Grauman, D. J., Blot, W. J., Pennello, G. A., Hoover, R. N., \& Fraumeni, J. F. (1999). Atlas of Cancer Mortality in the United States, 1950-94. Bethesda, MD. Retrieved from http://ratecalc.cancer.gov/archivedatlas/pdfs/text.pdf

Doescher, M. P., \& Jackson, J. E. (2009). Trends in Cervical and Breast Cancer Screening Practices Among Women in Rural and Urban Areas of the United States. Journal of Public Health Management Practice, 15(3), 200-209.

Dunne, E. F., Unger, E. R., Sternberg, M., McQuillan, G., Swan, D. C., Patel, S. S., \& Markowitz, L. E. (2007). Prevalence of HPV infection among females in the United States. JAMA, 297(8), 813-9. doi:10.1001/jama.297.8.813

Forman, D., de Martel, C., Lacey, C. J., Soerjomataram, I., Lortet-Tieulent, J., Bruni, L., ... Franceschi, S. (2012). Global burden of human papillomavirus and related diseases. Vaccine, 30(Suppl 5). doi:10.1016/j.vaccine.2012.07.055

Franco, E. L., Duarte-Franco, E., \& Ferenczy, A. (2001). Cervical cancer: Epidemiology, prevention, and the role of human papillomavirus. CMAJ, 164, 1017-25.

Ghanem, K. G., Hutton, H. E., Zenilman, J. M., Zimba, R., \& Erbelding, E. J. (2005). Audio computer assisted self interview and face to face interview modes in assessing response bias among STD clinic patients. Sexually Transmitted Infections, 81(5), 421-425. doi:10.1136/sti.2004.013193

Hariri, S., Unger, E. R., Sternberg, M., Dunne, E. F., Swan, D., Patel, S., \& Markowitz, L. E. (2011). Prevalence of genital human papillomavirus among females in the United States, the National Health and Nutrition Examination Survey, 2003 -2006. Journal of Infectious Diseases, 204, 566-573. doi:10.1093/infdis/jir341

Horner, M.-J., Altekruse, S. F., Zou, Z., Wideroff, L., Katki, H. A., \& Stinchcomb, D. G. (2011). U.S. geographic distribution of prevaccine era cervical cancer screening, incidence, stage , and mortality. Cancer Epidemiology, Biomarkers and Prevention, 20(4), 591-9. doi:10.1158/1055-9965.EPI-10-1183

Howlader, N., Noone, A. M., Krapcho, M., Garshell, J., Neyman, N., Altekruse, S. F., ... Cronin, K. A. (2013). SEER Cancer Statistics Review, 1975-2010. Bethesda, MD. doi:based on November 2012 SEER data submission, posted to the SEER web site, 
April 2013

International Agency for Research on Cancer. (2014). GLOBOCAN 2012: Estimated cancer incidence, mortality and prevalence worldwide in 2012. IARC CancerBase. Lyon, France. Retrieved from http://globocan.iarc.fr/Pages/fact_sheets_cancer.aspx

Kahn, J. A., Lan, D., \& Kahn, R. S. (2007). Sociodemographic factors associated with high-risk human papillomavirus infection. Obstetrics and Gynecology, 110(1), 8795.

Kelly, C. A., Soler-Hampejsek, E., Mensch, B. S., \& Hewett, P. C. (2013). Social desirability bias in sexual behavior reporting: Evidence from an interview mode experiment in rural Malawi. International Perspectives on Sexual and Reproductive Health, 39(1), 14-21. doi:10.1363/3901413

Markowitz, L. E., Hariri, S., Lin, C., Dunne, E. F., Steinau, M., McQuillan, G., \& Unger, E. R. (2013). Reduction in human papillomavirus (HPV) prevalence among young women following HPV vaccine introduction in the United States, National Health and Nutrition Examination Surveys, 2003-2010. The Journal of Infectious Diseases, 208(3), 385-93. doi:10.1093/infdis/jit192

Markowitz, L. E., Sternberg, M., Dunne, E. F., McQuillan, G., \& Unger, E. R. (2009). Seroprevalence of human papillomavirus types 6,11,16, and 18 in the United States: National Health and Nutrition Examination Survey 2003-2004. The Journal of Infectious Diseases, 200(7), 1059-67. doi:10.1086/604729

Muñoz, N., Bosch, F. X., de Sanjosé, S., Herrero, R., Castellsagué, X., Shah, K. V, ... Meijer, C. J. L. M. (2003). Epidemiologic classification of human papillomavirus types associated with cervical cancer. The New England Journal of Medicine, 348(6), 518-27. doi:10.1056/NEJMoa021641

National Institute of Health. (2015). Cancer trends progress report: cervical cancer screening. Retrieved April 8, 2016, from http://progressreport.cancer.gov/detection/cervical_cancer

O'Sullivan, L. F. (2008). Challenging Assumptions Regarding the Validity of Self-Report Measures: The Special Case of Sexual Behavior. Journal of Adolescent Health, 42(3), 207-208. doi:10.1016/j.jadohealth.2008.01.002

Ogoina, D., Musa, B. O., \& Onyemelukwe, G. C. (2013). Human papilloma virus (HPV) infection is associated with HIV-1 infection and AIDS in HIV-infected adult patients from Zaria, Northern Nigeria. The Pan African Medical Journal, 15, 38. doi:10.11604/pamj.2013.15.38.2349

Petignat, P., Faltin, D. L., Bruchim, I., Tramèr, M. R., Franco, E. L., \& Coutlée, F. (2007). Are self-collected samples comparable to physician-collected cervical specimens for human papillomavirus DNA testing ? A systematic review and metaanalysis. Gynecologic Oncology, 105, 530-535. doi:10.1016/j.ygyno.2007.01.023

Phillips, A. E., Gomez, G. B., Boily, M. C., \& Garnett, G. P. (2010). A systematic review 
and meta-analysis of quantitative interviewing tools to investigate self-reported HIV and STI associated behaviours in low- and middle-income countries. International Journal of Epidemiology, 39(6), 1541-1555. doi:10.1093/ije/dyq114

Reiter, P. L., Katz, M. L., Ruffin, M. T., Hade, E. M., Degraffenreid, C. R., Patel, D. a, ... Unger, E. R. (2013). HPV prevalence among women from Appalachia: results from the CARE Project. PloS One, 8(8), e74276. doi:10.1371/journal.pone.0074276

StataCorp. (2013). Stata Statistical Software: Release 13. College Station, Texas: StataCorp LP.

U.S. Department of Health and Human Services. (2014). Healthy People 2020. Washington, D.C. Retrieved from http://www.healthypeople.gov/2020/about/default.aspx

Wright, T. C., Denny, L., \& Kuhn, L. (2000). HPV DNA Testing of Self-collected Screening to Detect Cervical Cancer. JAMA, 283(1), 81-86.

Zhao, F., Lewkowitz, A. K., Chen, F., Lin, M. J., Hu, S., Zhang, X., ... Castle, P. E. (2012). Pooled analysis of a self-sampling HPV DNA test as a cervical cancer primary screening method. Journal of the National Cancer Institute, 104(3), 178188. doi:10.1093/jnci/djr532

Zipf, G., Chiappa, M., Porter, K., Ostchega, Y., Lewis, B., \& Dostal, J. (2013). National Health and Nutrition Examination Survey: plan and operations , 1999 - 2010. Vital and Health Statistics, 1(56), 10-22. Retrieved from

http://www.cdc.gov/nchs/data/series/sr_01/sr01_056.pdf

\section{Tables}

Table 1. Prevalence of high-risk oncogenic, oncogenic, and non-oncogenic types HPV

\begin{tabular}{|c|c|c|c|}
\hline \multirow[t]{2}{*}{ 14-26 year-old females } & \multicolumn{2}{|c|}{ Region $^{\mathrm{a}}$} & \multirow[t]{2}{*}{ p-value } \\
\hline & South $(n=532)$ & Other $^{b}(n=811)$ & \\
\hline High-risk oncogenic ${ }^{c}$ types infection & & & 0.15 \\
\hline Yes & $25.6(22.4-33.3)$ & $29.1(24.8-33.8)$ & \\
\hline No & $74.4(66.7-77.5)$ & $70.9(66.2-75.2)$ & \\
\hline Any oncogenic ${ }^{\mathrm{d}}$ types infection & & & 0.84 \\
\hline Yes & $31.7(26.4-37.6)$ & $32.3(27.9-36.9)$ & \\
\hline No & $68.3(62.4-73.6)$ & $67.7(63.0-72.1)$ & \\
\hline Non-oncogenic ${ }^{\mathrm{e}}$ types infection & & & 0.08 \\
\hline Yes & $36.5(30.0-43.4)$ & $31.9(26.9-37.4)$ & \\
\hline No & $63.4(56.6-69.9)$ & $68.1(62.6-73.1)$ & \\
\hline 27-59 year-old females & South $(n=1,066)$ & Other $^{b}(n=1841)$ & \\
\hline
\end{tabular}




\begin{tabular}{rrrrr}
\hline \multicolumn{1}{l}{ High-risk oncogenic ${ }^{\mathrm{c}}$ types infection } & & $\mathbf{0 . 0 0 0 1}$ \\
\hline & Yes & $20.9(17.4-24.9)$ & $14.5(12.9-16.3)$ & \\
\hline No & $79.1(75.1-82.6)$ & $85.5(83.7-87.1)$ & \\
\hline Any oncogenic ${ }^{\mathrm{d}}$ types infection & & & & $\mathbf{0 . 0 0 0 1}$ \\
\hline & Yes & $24.0(19.9-28.7)$ & $17.9(16.3-19.5)$ & \\
\hline No & $76.0(71.3-80.1)$ & $82.1(80.5-83.7)$ & \\
\hline Non-oncogenic ${ }^{\mathrm{e}}$ types infection & & & & $\mathbf{0 . 0 1 4}$ \\
\hline Yes & $32.4(29.6-35.4)$ & $28.1(26.3-30.1)$ & \\
\hline No & $67.6(64.6-70.4)$ & $71.9(69.9-73.7)$ & \\
\hline
\end{tabular}

${ }^{a}$ To prevent potential disclosure, the region rather than the state of residence was included in the dataset

${ }^{\mathrm{b}}$ Northeast, West, and Midwest combined

${ }^{\mathrm{c}} \mathrm{HPV}$ types: $16,18,31,33,35,39,45,51,52,56,58$, and 59

${ }^{\mathrm{d}}$ HPV types: 6, 11, 16, 18, 31, 33, 35, 39, 40, 42, 45, 51, 52, 53, 55, 56, 58, and 59

eAll other HPV types

Table 2. Socio-demographic and behavioral characteristics associated with infection from high-risk types among 14-26 year-old females

\begin{tabular}{|c|c|c|c|c|c|c|}
\hline \multirow{4}{*}{ Characteristics $^{\mathrm{a}}$} & \multicolumn{6}{|c|}{ HR Oncogenic ${ }^{\mathrm{a}}$ types } \\
\hline & \multicolumn{3}{|c|}{ South $(n=532)$} & \multicolumn{3}{|c|}{ Other $^{\mathrm{b}}$ regions $(\mathrm{n}=811)$} \\
\hline & \multicolumn{2}{|c|}{$\begin{array}{l}\text { Infection } \\
\text { Status }\end{array}$} & \multirow[t]{2}{*}{ p-value } & \multicolumn{2}{|c|}{$\begin{array}{c}\text { Infection } \\
\text { Status }\end{array}$} & \multirow[t]{2}{*}{$\mathrm{p}$-value } \\
\hline & Yes & No & & Yes & No & \\
\hline Race/Ethnicity & $\%$ & $\%$ & 0.04 & $\%$ & $\%$ & 0.2 \\
\hline Non-Hispanic White & 44.2 & 55.7 & & 65.6 & 63.0 & \\
\hline Non-Hispanic Black & 34.2 & 19.0 & & 11.4 & 8.9 & \\
\hline Other & 21.6 & 25.3 & & 23.0 & 28.1 & \\
\hline Country of birth & & & 0.28 & & & 0.7 \\
\hline US & 89.9 & 88.0 & & 86.5 & 88.4 & \\
\hline Other & 10.1 & 12.0 & & 13.5 & 11.6 & \\
\hline Country of citizenship & & & 0.58 & & & 0.9 \\
\hline US & 93.8 & 92.0 & & 91.7 & 91.6 & \\
\hline Other & 6.2 & 7.4 & & 8.3 & 8.4 & \\
\hline Marital Status & & & 0.5 & & & 0.00001 \\
\hline Married & 22.5 & 31.3 & & 4.8 & 25.2 & \\
\hline Never married 2 & 65.3 & 65.3 & & 69.3 & 53.2 & \\
\hline
\end{tabular}




\begin{tabular}{|c|c|c|c|c|c|c|}
\hline Divorced/separated/cohabitating & 12.2 & 13.1 & & 25.9 & 21.6 & \\
\hline Income to federal poverty level & & & 0.45 & & & 0.03 \\
\hline$<100 \%$ & 35.6 & 25.2 & & 26.1 & 24.4 & \\
\hline $100-299 \%$ & 22.8 & 25.5 & & 23.6 & 20.1 & \\
\hline $300-499 \%$ & 27.4 & 30.8 & & 29.1 & 24.4 & \\
\hline$\geq 500 \%$ & 14.2 & 18.5 & & 21.2 & 31.1 & \\
\hline Health insurance & & & 0.55 & & & 0.036 \\
\hline Yes & 69.1 & 74.3 & & 68.3 & 79.3 & \\
\hline No & 30.9 & 25.7 & & 31.7 & 20.7 & \\
\hline $\begin{array}{l}\text { Type of place most often go for } \\
\text { healthcare }\end{array}$ & & & 0.39 & & & 0.7 \\
\hline Doctor's office & 60.3 & 66.9 & & 74.0 & 71.8 & \\
\hline Other & 39.7 & 33.1 & & 26.0 & 28.2 & \\
\hline $\begin{array}{l}\text { No. of times receive healthcare } \\
\text { last year }\end{array}$ & & & 0.69 & & & 0.8 \\
\hline None & 10.4 & 13.2 & & 12.2 & 10.8 & \\
\hline$\leq 3$ & 57.2 & 52.2 & & 50.1 & 52.2 & \\
\hline$\geq 4$ & 32.4 & 34.6 & & 37.7 & 37.0 & \\
\hline Ever taken birth control pills & & & 0.005 & & & 0.0001 \\
\hline Yes & 70.2 & 45.6 & & 62.2 & 42.5 & \\
\hline No & 29.8 & 54.4 & & 37.8 & 57.5 & \\
\hline $\begin{array}{l}\text { No. of years of birth control } \\
\text { pills }\end{array}$ & & & 0.51 & & & 0.5 \\
\hline$<2$ & 43.2 & 47.6 & & 44.4 & 50.8 & \\
\hline$\geq 2$ & 56.8 & 52.4 & & 55.6 & 49.2 & \\
\hline Ever had sex ${ }^{e}$ & & & 0.00001 & & & 0.00001 \\
\hline Yes & 95.5 & 67.1 & & 95.0 & 62.7 & \\
\hline No & 4.5 & 32.9 & & 5.0 & 37.3 & \\
\hline Age at first sexual intercourse & & & 0.96 & & & 0.6 \\
\hline $9-14$ years & 23.8 & 23.9 & & 25.5 & 22.4 & \\
\hline$>=15$ years & 76.2 & 76.1 & & 74.5 & 77.6 & \\
\hline No. of lifetime sex partners & & & 0.00001 & & & 0.00001 \\
\hline$\leq 3$ & 20.5 & 66.3 & & 27.3 & 64.7 & \\
\hline$\geq 4$ & 79.5 & 33.7 & & 72.7 & 35.3 & \\
\hline $\begin{array}{l}\text { No. of sex partners } 5+\text { years } \\
\text { older }\end{array}$ & & & 0.52 & & & 0.002 \\
\hline None & 71.1 & 75.8 & & 59.0 & 75.9 & \\
\hline$\geq 1$ & 28.9 & 24.2 & & 41.0 & 24.1 & \\
\hline
\end{tabular}


${ }^{\mathrm{a}} \mathrm{HPV}$ types: $16,18,31,33,35,39,45,51,52,56,58$, and 59

${ }^{\mathrm{b}}$ Northeast, West, and Midwest combined

${ }^{\text {a}} H P V$ types: 16, 18, 31, 33, 35, 39, 45, 51, 52, 56, 58, and 59

${ }^{\mathrm{b}} \mathrm{HPV}$ types: 6, 11, 16, 18, 31, 33, 35, 39, 40, 42, 45, 51, 52, 53, 55, 56, 58, and 59

${ }^{\mathrm{c}}$ Northeast, West, and Midwest combined

Table 3. Socio-demographic and behavioral characteristics associated with infection from any oncogenic types among 14-26 year-old females

\begin{tabular}{|c|c|c|c|c|c|c|}
\hline \multirow{4}{*}{ Characteristics $^{\mathbf{a}}$} & \multicolumn{6}{|c|}{ Any oncogenic ${ }^{a}$ HPV types } \\
\hline & \multicolumn{3}{|c|}{ South $(n=532)$} & \multicolumn{3}{|c|}{ Other $^{b}$ regions $(n=811)$} \\
\hline & \multicolumn{2}{|c|}{$\begin{array}{l}\text { Infection } \\
\text { Status }\end{array}$} & \multirow[t]{2}{*}{ p-value } & \multicolumn{2}{|c|}{$\begin{array}{c}\text { Infection } \\
\text { Status }\end{array}$} & \multirow[t]{2}{*}{$\mathrm{p}$-value } \\
\hline & Yes & No & & Yes & No & \\
\hline Race/Ethnicity & $\%$ & $\%$ & 0.015 & $\%$ & $\%$ & 0.14 \\
\hline Non-Hispanic White & 43.6 & 56.7 & & 65.6 & 62.8 & \\
\hline Non-Hispanic Black & 34.4 & 18.0 & & 11.7 & 8.7 & \\
\hline Other & 22.0 & 25.3 & & 22.7 & 28.5 & \\
\hline Country of birth & & & 0.15 & & & 0.8 \\
\hline US & 90.8 & 87.4 & & 87.4 & 88.0 & \\
\hline Other & 9.2 & 12.8 & & 12.6 & 12.0 & \\
\hline Country of citizenship & & & & & & 0.6 \\
\hline US & 94.4 & 92.2 & 0.35 & 92.3 & 91.3 & \\
\hline Other & 5.6 & 7.8 & & 7.7 & 8.7 & \\
\hline Marital Status & & & 0.5 & & & 0.00001 \\
\hline Married & 23.4 & 31.6 & & 5.1 & 26.7 & \\
\hline Never married & 64.2 & 55.3 & & 69.4 & 51.7 & \\
\hline Divorced/separated/cohabitating & 12.4 & 13.1 & & 25.5 & 21.6 & \\
\hline Income to federal poverty level & & & 0.3 & & & 0.3 \\
\hline$<100 \%$ & 36.2 & 24.3 & & 26.6 & 24.0 & \\
\hline $100-299 \%$ & 21.9 & 26.1 & & 23.8 & 19.8 & \\
\hline $300-499 \%$ & 29.6 & 29.9 & & 27.5 & 25.0 & \\
\hline$\geq 500 \%$ & 12.3 & 19.7 & & 22.1 & 31.2 & \\
\hline Health insurance & & & 0.6 & & & 0.02 \\
\hline Yes & 70.3 & & & 67.6 & 80.2 & \\
\hline No & 29.7 & 74.0 & & 32.4 & 19.8 & \\
\hline $\begin{array}{l}\text { Type of place most often go for } \\
\text { healthcare }\end{array}$ & & 26.0 & 0.13 & & & 0.8 \\
\hline
\end{tabular}




\begin{tabular}{|c|c|c|c|c|c|c|}
\hline Doctor's office & 56.6 & 69.0 & & 71.6 & 72.8 & \\
\hline Other & 43.4 & 31.0 & & 28.4 & 27.2 & \\
\hline $\begin{array}{l}\text { No. of times receive healthcare } \\
\text { last year }\end{array}$ & & & 0.92 & & & 0.5 \\
\hline None & 11.9 & 12.7 & & 12.9 & 10.3 & \\
\hline$\leq 3$ & 54.7 & 53.1 & & 48.8 & 53.0 & \\
\hline$\geq 4$ & 33.4 & 34.2 & & 38.3 & 36.7 & \\
\hline Ever taken birth control pills & & & 0.0005 & & & 0.0001 \\
\hline Yes & 69.8 & 44.4 & & 63.3 & 41.2 & \\
\hline No & 30.2 & 55.6 & & 36.7 & 58.8 & \\
\hline $\begin{array}{l}\text { No. of years of birth control } \\
\text { pills }\end{array}$ & & & 0.11 & & & 0.8 \\
\hline$<2$ & 41.8 & 48.9 & & 44.2 & 51.4 & \\
\hline$\geq 2$ & 58.2 & 51.1 & & 55.8 & 48.6 & \\
\hline Ever had sex ${ }^{e}$ & & & 0.00001 & & & 0.00001 \\
\hline Yes & 95.2 & 65.7 & & 95.4 & 61.2 & \\
\hline No & 4.8 & 34.3 & & 4.6 & 38.8 & \\
\hline Age at first sexual intercourse & & & 0.8 & & & 0.5 \\
\hline 9-14 years & 23.1 & 24.3 & & 25.4 & 22.2 & \\
\hline$>=15$ years & 76.9 & 75.7 & & 74.6 & 77.8 & \\
\hline No. of lifetime sex partners & & & 0.00001 & & & 0.00001 \\
\hline$\leq 3$ & 22.0 & 68.9 & & 27.0 & 67.0 & \\
\hline$\geq 4$ & 78.0 & 31.1 & & 73.0 & 33.0 & \\
\hline $\begin{array}{l}\text { No. of sex partners } 5+\text { years } \\
\text { older }\end{array}$ & & & 0.08 & & & 0.001 \\
\hline None & 68.3 & 78.1 & & 59.2 & 76.9 & \\
\hline$\geq 1$ & 31.7 & 21.9 & & 40.8 & 23.1 & \\
\hline
\end{tabular}

${ }^{\mathrm{a}} \mathrm{HPV}$ types: $6,11,16,18,31,33,35,39,40,42,45,51,52,53,55,56,58$, and 59

${ }^{b}$ Northeast, West, and Midwest combined 
Table 4. Socio-demographic and behavioral characteristics associated with infection from high-risk oncogenic types among $27-59$ year-old females

\begin{tabular}{|c|c|c|c|c|c|c|}
\hline \multirow{4}{*}{ Characteristics } & \multicolumn{6}{|c|}{ HR Oncogenic ${ }^{\mathrm{a}}$ types } \\
\hline & \multicolumn{3}{|c|}{ South $(n=1,066)$} & \multicolumn{3}{|c|}{ Other regions $(\mathrm{n}=1841)$} \\
\hline & \multicolumn{2}{|c|}{$\begin{array}{l}\text { Infection } \\
\text { Status }\end{array}$} & \multirow[t]{2}{*}{ p-value } & \multicolumn{2}{|c|}{$\begin{array}{l}\text { Infection } \\
\text { Status }\end{array}$} & \multirow[t]{2}{*}{$\mathrm{p}$-value } \\
\hline & Yes & No & & Yes & No & \\
\hline Race/Ethnicity & $\%$ & $\%$ & 0.017 & $\%$ & $\%$ & 0.004 \\
\hline Non-Hispanic White & 57.9 & 59.0 & & 64.8 & 73.1 & \\
\hline Non-Hispanic Black & 26.1 & 18.9 & & 13.8 & 7.3 & \\
\hline Other & 16.0 & 22.1 & & 21.5 & 19.6 & \\
\hline Country of birth & & & 0.04 & & & 0.9 \\
\hline US & 91.0 & 83.2 & & 82.4 & 82.1 & \\
\hline Other & 9.0 & 16.8 & & 17.6 & 17.9 & \\
\hline Country of citizenship & & & 0.06 & & & 0.6 \\
\hline US & 93.5 & 90.6 & & 91.3 & 90.2 & \\
\hline Other & 6.5 & 9.4 & & 8.7 & 9.2 & \\
\hline Marital Status & & & $\begin{array}{r}0.0000 \\
1\end{array}$ & & & 0.00001 \\
\hline Married & 38.1 & 66.6 & & 36.1 & 65.9 & \\
\hline Never married & 18.4 & 8.9 & & 20.3 & 11.4 & \\
\hline Divorced/separated/cohabitati & 43.5 & 24.5 & & 43.6 & 22.7 & \\
\hline $\begin{array}{l}\text { Income to federal poverty } \\
\text { level }\end{array}$ & & & 0.008 & & & 0.0002 \\
\hline$<100 \%$ & 27.9 & 16.8 & & 21.9 & 10.9 & \\
\hline $100-299 \%$ & 22.3 & 21.5 & & 22.1 & 16.6 & \\
\hline $300-499 \%$ & 27.7 & 26.8 & & 19.9 & 28.9 & \\
\hline$\geq 500 \%$ & 22.1 & 34.9 & & 36.1 & 43.6 & \\
\hline Health insurance & & & 0.006 & & & 0.02 \\
\hline Yes & 64.2 & 76.9 & & 79.2 & 85.6 & \\
\hline No & 35.8 & 23.1 & & 20.8 & 14.4 & \\
\hline $\begin{array}{l}\text { Type of place most often go } \\
\text { for healthcare }\end{array}$ & & & 0.7 & & & 0.01 \\
\hline Doctor's office & 76.1 & 77.5 & & 71.0 & 77.3 & \\
\hline Other & 23.9 & 22.5 & & 29.0 & 22.7 & \\
\hline $\begin{array}{l}\text { No. of healthcare visits last } \\
\text { year }\end{array}$ & & & 0.5 & & & 0.041 \\
\hline None & 14.9 & 12.0 & & 13.3 & 12.0 & \\
\hline
\end{tabular}




\begin{tabular}{|c|c|c|c|c|c|c|}
\hline$\leq 3$ & 47.8 & 48.8 & & 55.5 & 48.5 & \\
\hline$\geq 4$ & 37.3 & 39.2 & & 31.2 & 39.5 & \\
\hline Ever taken birth control pills & & & 0.97 & & & 0.43 \\
\hline Yes & 83.7 & 83.6 & & 84.8 & 82.8 & \\
\hline No & 16.3 & 16.4 & & 15.2 & 17.2 & \\
\hline $\begin{array}{l}\text { No. of years of birth control } \\
\text { pills }\end{array}$ & & & 0.15 & & & 0.04 \\
\hline$<2$ & 30.6 & 25.0 & & 34.1 & 25.2 & \\
\hline$\geq 2$ & 69.4 & 75.0 & & 65.9 & 74.8 & \\
\hline Ever had sex $\mathrm{e}^{\mathrm{e}}$ & & & 0.15 & & & 0.14 \\
\hline Yes & 98.2 & 98.3 & & 98.8 & 97.7 & \\
\hline No & 1.8 & 1.8 & & 1.2 & 2.3 & \\
\hline Age at first sexual intercourse & & & 0.47 & & & 0.016 \\
\hline $9-14$ years & 17.3 & 13.9 & & 14.1 & 9.9 & \\
\hline$>=15$ years & 82.7 & 86.1 & & 85.9 & 90.1 & \\
\hline No. of lifetime sex partners & & & 0.0004 & & & 0.00001 \\
\hline$\leq 3$ & 21.8 & 38.7 & & 20.3 & 41.4 & \\
\hline$\geq 4$ & 78.2 & 61.3 & & 79.7 & 58.6 & \\
\hline $\begin{array}{l}\text { No. of sex partners } 5+\text { years } \\
\text { older }\end{array}$ & & & 0.17 & & & 0.0002 \\
\hline None & 58.1 & 72.7 & & 58.4 & 75.2 & \\
\hline$\geq 1$ & 41.9 & 27.3 & & 41.6 & 24.8 & \\
\hline
\end{tabular}

${ }^{\text {a}} \mathrm{HPV}$ types: $16,18,31,33,35,39,45,51,52,56,58$, and 59

${ }^{b}$ Northeast, West, and Midwest combined

Table 5. Socio-demographic and behavioral characteristics associated with infection from any oncogenic types among 27-59 year-old females

Any oncogenic ${ }^{\mathrm{a}}$ HPV types HPV

\begin{tabular}{|c|c|c|c|c|c|c|}
\hline \multirow[t]{3}{*}{ Characteristics } & \multicolumn{3}{|c|}{ South $(n=1,066)$} & \multicolumn{3}{|c|}{ Other $^{\mathrm{b}}$ regions $(\mathrm{n}=1,841)$} \\
\hline & \multicolumn{2}{|c|}{$\begin{array}{l}\text { Infection } \\
\text { Status }\end{array}$} & \multirow[t]{2}{*}{ p-value } & \multicolumn{2}{|c|}{$\begin{array}{l}\text { Infection } \\
\text { Status }\end{array}$} & \multirow[t]{2}{*}{$\mathrm{p}$-value } \\
\hline & Yes & No & & Yes & No & \\
\hline Race/Ethnicity & $\%$ & $\%$ & 0.02 & $\%$ & $\%$ & 0.015 \\
\hline Non-Hispanic White & 56.9 & 59.4 & & 66.4 & 73.1 & \\
\hline Non-Hispanic Black & 25.9 & 18.6 & & 12.5 & 7.3 & \\
\hline Other & 17.2 & 22.0 & & 21.2 & 19.6 & \\
\hline Country of birth & & & 0.02 & & & 0.7 \\
\hline US & 90.8 & 82.9 & & 83.7 & 81.9 & \\
\hline
\end{tabular}




\begin{tabular}{|c|c|c|c|c|c|c|}
\hline Other & 9.2 & 17.1 & & 16.3 & 18.1 & \\
\hline Country of citizenship & & & 0.15 & & & 0.32 \\
\hline US & 93.3 & 90.5 & & 92.0 & 90.0 & \\
\hline Other & 6.7 & 9.5 & & 8.0 & 10.0 & \\
\hline Marital Status & & & .0000 & & & 0.00001 \\
\hline & & & 1 & & & \\
\hline Married & 39.6 & 67.4 & & 40.1 & 66.2 & \\
\hline Never married & 18.1 & 8.6 & & 17.4 & 11.7 & \\
\hline Divorced/separated/cohabitati & 42.3 & 24.0 & & 42.4 & 22.1 & \\
\hline ng & & & & & & \\
\hline $\begin{array}{l}\text { Income to federal poverty } \\
\text { level }\end{array}$ & & & 0.006 & & & 0.001 \\
\hline$<100 \%$ & 27.2 & 16.5 & & 20.0 & 10.9 & \\
\hline $100-299 \%$ & 23.3 & 21.2 & & 21.3 & 16.5 & \\
\hline $300-499 \%$ & 28.1 & 26.6 & & 21.3 & 29.0 & \\
\hline$\geq 500 \%$ & 21.4 & 35.6 & & 37.4 & 43.6 & \\
\hline Health insurance & & & 0.004 & & & 0.05 \\
\hline Yes & 64.0 & 77.5 & & 80.8 & 85.5 & \\
\hline No & 36.0 & 22.5 & & 19.2 & 14.5 & \\
\hline $\begin{array}{l}\text { Type of place most often go } \\
\text { for healthcare }\end{array}$ & & & 0.4 & & & 0.01 \\
\hline Doctor's office & 74.8 & 77.9 & & 71.6 & 77.5 & \\
\hline Other & 25.2 & 22.1 & & 28.4 & 22.5 & \\
\hline $\begin{array}{l}\text { No. of healthcare visits last } \\
\text { year }\end{array}$ & & & 0.8 & & & 0.4 \\
\hline None & 13.6 & 12.3 & & 12.4 & 12.2 & \\
\hline$\leq 3$ & 48.5 & 48.6 & & 52.9 & 48.7 & \\
\hline$\geq 4$ & 37.9 & 39.1 & & 34.8 & 39.1 & \\
\hline Ever taken birth control pills & & & 0.9 & & & 0.9 \\
\hline Yes & 83.2 & 83.7 & & 83.3 & 83.1 & \\
\hline No & 16.8 & 16.3 & & 16.7 & 16.9 & \\
\hline $\begin{array}{l}\text { No. of years of birth control } \\
\text { pills }\end{array}$ & & & 0.2 & & & 0.08 \\
\hline$<2$ & 30.4 & 24.9 & & 32.6 & 25.2 & \\
\hline$\geq 2$ & 69.6 & 75.1 & & 67.4 & 74.8 & \\
\hline Ever had sex ${ }^{\mathrm{e}}$ & & & 0.5 & & & 0.2 \\
\hline Yes & 97.9 & 97.4 & & 98.6 & 97.7 & \\
\hline No & 2.1 & 2.6 & & 1.4 & 2.3 & \\
\hline Age at first sexual intercourse & & & 0.3 & & & 0.0003 \\
\hline 9-14 years & 18.0 & 13.5 & & 15.5 & 90.6 & \\
\hline$>=15$ years & 82.0 & 86.5 & & 84.5 & 9.2 & \\
\hline
\end{tabular}


No. of lifetime sex partners

\begin{tabular}{lllllll}
$\leq 3$ & 21.7 & 39.4 & & $\mathbf{0 . 0 0 4}$ & & $\mathbf{0 . 0 0 0 0 1}$ \\
$\geq 4$ & 78.3 & 60.6 & & 78.4 & 58.0 & \\
\hline
\end{tabular}

No. of sex partners $5+$ years 0.3

$\mathbf{0 . 0 0 3}$

older

$\begin{array}{rrrrr}\text { None } & 62.0 & 71.9 & 60.2 & 75.6 \\ \geq 1 & 38.0 & 28.1 & 39.8 & 24.4\end{array}$

${ }^{\mathrm{a}} \mathrm{HPV}$ types: $6,11,16,18,31,33,35,39,40,42,45,51,52,53,55,56,58$, and 59

${ }^{b}$ Northeast, West, and Midwest combined

Table 6. Odds of HPV infections among sexually active 14-26 year-old females by socio-demographic and sexual behavioral characteristics

\begin{tabular}{|c|c|c|c|c|}
\hline \multirow[t]{3}{*}{ Characteristics } & \multicolumn{2}{|c|}{ HR $^{\mathrm{a}}$ onocogenic types HPV } & \multicolumn{2}{|c|}{ Any oncogenic ${ }^{\mathrm{b}}$ types HPV } \\
\hline & South & Other ${ }^{\mathrm{c}}$ & South & Other ${ }^{\mathrm{c}}$ \\
\hline & $\mathrm{aOR}^{\mathrm{d}}$ & $\mathrm{aOR}$ & $\mathrm{aOR}$ & $\mathrm{aOR}$ \\
\hline \multicolumn{5}{|l|}{ Race/Ethnicity } \\
\hline Non-Hispanic White & Ref. & Ref. & Ref. & Ref. \\
\hline Non-Hispanic Black & $\begin{array}{c}1.79(0.62- \\
5.2)\end{array}$ & $\begin{array}{c}0.85(0.35- \\
2.1)\end{array}$ & $\begin{array}{c}1.73(0.74- \\
4.0)\end{array}$ & $\begin{array}{c}1.09(0.47- \\
2.6)\end{array}$ \\
\hline Other & $\begin{array}{c}1.21(0.56- \\
2.6)\end{array}$ & $\begin{array}{c}0.73(0.39- \\
1.4)\end{array}$ & $\begin{array}{c}1.32(0.60- \\
2.94)\end{array}$ & $\begin{array}{c}0.69(0.40- \\
1.19)\end{array}$ \\
\hline \multicolumn{5}{|l|}{ Marital Status } \\
\hline Married & Ref. & Ref. & Ref. & Ref. \\
\hline Never married & $\begin{array}{c}1.56(0.44- \\
5.5)\end{array}$ & $\begin{array}{l}12.79(4.8- \\
34.1)^{* * * *}\end{array}$ & $\begin{array}{c}1.33(0.18- \\
1.4)\end{array}$ & $\begin{array}{l}14.1(6.3- \\
31.4)^{* * * *}\end{array}$ \\
\hline $\begin{array}{r}\text { Divorced/separated/co } \\
\text { habitating }\end{array}$ & $\begin{array}{c}1.62(0.47- \\
5.5)\end{array}$ & $\begin{array}{c}6.09 \\
(2.2=16.6)^{* *} \\
*\end{array}$ & $\begin{array}{c}1.41(0.36- \\
5.5)\end{array}$ & $\begin{array}{l}6.13(2.4- \\
15.8)^{* * *}\end{array}$ \\
\hline \multicolumn{5}{|l|}{ Federal poverty level } \\
\hline$<100 \%$ & Ref. & Ref. & Ref. & Ref. \\
\hline $100-299 \%$ & $\begin{array}{c}0.59(0.25- \\
1.4)\end{array}$ & $\begin{array}{c}1.79(0.51- \\
6.2)\end{array}$ & $\begin{array}{c}0.50(0.17- \\
1.4)\end{array}$ & $\begin{array}{c}2.18(0.59- \\
8.0)\end{array}$ \\
\hline $300-499 \%$ & $\begin{array}{c}0.36(0.17- \\
0.75)^{* *}\end{array}$ & $\begin{array}{c}1.59(0.65- \\
3.9)\end{array}$ & $\begin{array}{c}0.41(0.17- \\
0.99)^{*}\end{array}$ & $\begin{array}{c}1.32(0.57- \\
3.1)\end{array}$ \\
\hline$\geq 500 \%$ & $\begin{array}{c}0.52(0.13- \\
2.2)\end{array}$ & $\begin{array}{c}1.01(0.31- \\
3.2)\end{array}$ & $\begin{array}{c}0.36(0.07- \\
1.9)\end{array}$ & $\begin{array}{c}1.16(0.41- \\
3.3)\end{array}$ \\
\hline \multicolumn{5}{|l|}{ Health insurance } \\
\hline No & Ref. & Ref. & Ref. & Ref. \\
\hline Yes & $\begin{array}{c}2.17(0.79- \\
5.9)\end{array}$ & $\begin{array}{c}0.66(0.27- \\
1.6)\end{array}$ & $\begin{array}{c}2.48(1.23- \\
5.02)^{* *}\end{array}$ & $\begin{array}{c}0.60(0.24- \\
1.51)\end{array}$ \\
\hline
\end{tabular}


No. of sex partners 5+ years older

$\begin{array}{rcccc}\text { None } & \text { Ref. } & \text { Ref. } & \text { Ref. } & \text { Ref. } \\ \geq 1 & 0.65(0.24- & 1.32(0.66- & 0.94(0.44- & 1.43(0.68- \\ & 1.8) & 2.7) & 1.97) & 3.0)\end{array}$

No. of lifetime sex

partners

$\begin{array}{ccccc}\leq 3 & \text { Ref. } & \text { Ref. } & \text { Ref. } & \text { Ref. } \\ \geq 4 & 8.27(2.8- & 4.03(2.2- & 8.51(3.7- & 4.40(2.4- \\ & 24.4)^{* * *} & 7.4)^{* * *} & 19.6)^{* * *} & 8.2)^{* * *}\end{array}$

${ }^{\mathrm{a}} \mathrm{HPV}$ types: $16,18,31,33,35,39,45,51,52,56,58$, and 59

${ }^{\mathrm{b}} \mathrm{HPV}$ types: 6, 11, 16, 18, 31, 33, 35, 39, 40, 42, 45, 51, 52, 53, 55, 56, 58, and 59

'Northeast, West, and Midwest combined

$\mathrm{d}_{\text {adjusted odds ratio }}$

$* \mathrm{p}<0.05 ; * * \mathrm{p}<0.01 ; * * * \mathrm{p}<0.001$

Table 7. Odds of HPV infections among sexually active 27-59 year-old females by socio-demographic and sexual behavioral characteristics

\begin{tabular}{|c|c|c|c|c|}
\hline \multirow[t]{3}{*}{ Characteristics } & \multicolumn{2}{|c|}{ HR $^{\mathrm{a}}$ oncogenic types HPV } & \multicolumn{2}{|c|}{ Any oncogenic ${ }^{b}$ types HPV } \\
\hline & South & Other $^{\mathrm{c}}$ & South & Other $^{\mathrm{c}}$ \\
\hline & $\mathrm{aOR}^{\mathrm{d}}$ & $\mathrm{aOR}^{\mathrm{d}}$ & $\mathrm{aOR}^{\mathrm{d}}$ & $\mathrm{aOR}^{\mathrm{d}}$ \\
\hline \multicolumn{5}{|l|}{ Race/Ethnicity } \\
\hline Non-Hispanic White & Ref. & Ref. & Ref. & Ref. \\
\hline Non-Hispanic Black & $\begin{array}{c}0.72(0.32- \\
1.7)\end{array}$ & $\begin{array}{c}0.63(0.25- \\
1.6)\end{array}$ & $\begin{array}{l}0.88(0.36- \\
2.1)\end{array}$ & $\begin{array}{c}0.52(0.22- \\
1.2)\end{array}$ \\
\hline Other & $\begin{array}{c}0.57(0.24- \\
1.3)\end{array}$ & $\begin{array}{c}0.76(0.31- \\
1.8)\end{array}$ & $\begin{array}{c}0.71(0.39- \\
1.29)\end{array}$ & $\begin{array}{c}0.78(0.36- \\
1.7)\end{array}$ \\
\hline \multicolumn{5}{|l|}{ Marital Status } \\
\hline Married & Ref. & Ref. & Ref. & Ref. \\
\hline Never married & $\begin{array}{l}5.14(1.7- \\
15.2)^{* *}\end{array}$ & $\begin{array}{c}2.38(1.23- \\
4.6) * *\end{array}$ & $\begin{array}{l}4.27(1.44- \\
12.6)^{* *}\end{array}$ & $\begin{array}{l}2.20(1.34- \\
3.61)^{* *}\end{array}$ \\
\hline $\begin{array}{r}\text { Divorced/separated/coh } \\
\text { abitating }\end{array}$ & $\begin{array}{l}2.98(0.98- \\
9.1)\end{array}$ & $\begin{array}{c}4.26(1.37- \\
13.3)^{*}\end{array}$ & $\begin{array}{c}2.09(0.69- \\
7.5)\end{array}$ & $\begin{array}{l}4.16(1.76- \\
9.8)^{* *}\end{array}$ \\
\hline \multicolumn{5}{|l|}{ Federal poverty level } \\
\hline$<100 \%$ & Ref. & Ref. & Ref. & Ref. \\
\hline $100-299 \%$ & $\begin{array}{l}0.57(0.15- \\
2.2)\end{array}$ & $\begin{array}{l}0.51(0.19- \\
1.4)\end{array}$ & $\begin{array}{l}0.56(0.15- \\
2.2)\end{array}$ & $\begin{array}{c}0.58(0.26- \\
1.3)\end{array}$ \\
\hline $300-499 \%$ & $\begin{array}{c}1.12(0.52- \\
2.4)\end{array}$ & $\begin{array}{c}0.26(0.10- \\
0.66)^{* *}\end{array}$ & $\begin{array}{c}1.32(0.50- \\
3.5)\end{array}$ & $\begin{array}{c}0.34(0.15- \\
0.78)^{*}\end{array}$ \\
\hline
\end{tabular}




\begin{tabular}{|c|c|c|c|c|}
\hline$\geq 500 \%$ & $\begin{array}{c}0.79(0.33- \\
1.9)\end{array}$ & $\begin{array}{c}0.57(0.25- \\
1.28)\end{array}$ & $\begin{array}{c}0.80(0.38- \\
1.7)\end{array}$ & $\begin{array}{c}0.69(0.31- \\
1.5)\end{array}$ \\
\hline \multicolumn{5}{|l|}{ Health insurance } \\
\hline No & Ref. & Ref. & Ref. & Ref. \\
\hline Yes & $\begin{array}{l}0.58(0.27- \\
1.2)\end{array}$ & $\begin{array}{l}0.92(0.30- \\
2.78)\end{array}$ & $\begin{array}{l}0.45(0.20- \\
1.02)\end{array}$ & $\begin{array}{l}0.97(0.32- \\
2.9)\end{array}$ \\
\hline \multicolumn{5}{|l|}{$\begin{array}{l}\text { No. of sex partners } 5+ \\
\text { years older }\end{array}$} \\
\hline None & Ref. & Ref. & Ref. & Ref. \\
\hline$\geq 1$ & $\begin{array}{c}1.92(0.45- \\
8.3)\end{array}$ & $\begin{array}{c}1.84(1.13- \\
3.0)^{* *}\end{array}$ & $\begin{array}{c}1.64(0.40- \\
7.1)\end{array}$ & $\begin{array}{l}1.57(0.92- \\
2.6)\end{array}$ \\
\hline \multicolumn{5}{|l|}{$\begin{array}{l}\text { No. of lifetime sex } \\
\text { partners }\end{array}$} \\
\hline$\leq 3$ & Ref. & Ref. & Ref. & Ref. \\
\hline$\geq 4$ & $\begin{array}{c}1.32(0.98- \\
2.23)\end{array}$ & $\begin{array}{l}3.78(1.6- \\
9.0)^{* *}\end{array}$ & $\begin{array}{c}1.43(0.8- \\
2.53) \\
\end{array}$ & $\begin{array}{l}3.9(1.9- \\
8.0) * * *\end{array}$ \\
\hline \multicolumn{5}{|l|}{$\begin{array}{l}\text { Age at first sexual } \\
\text { intercourse }\end{array}$} \\
\hline $9-14$ years & Ref. & Ref. & Ref. & Ref. \\
\hline$\geq 15$ years & $\begin{array}{l}1.26(0.5- \\
3.2)\end{array}$ & $\begin{array}{c}0.87(0.59- \\
1.3)\end{array}$ & $\begin{array}{c}.07(0.42- \\
2.74)\end{array}$ & $\begin{array}{c}0.47(0.24- \\
0.93)^{*}\end{array}$ \\
\hline
\end{tabular}

${ }^{\text {a}} \mathrm{HPV}$ types: $16,18,31,33,35,39,45,51,52,56,58$, and 59

${ }^{\mathrm{b}} \mathrm{HPV}$ types: 6, 11, 16, 18, 31, 33, 35, 39, 40, 42, 45, 51, 52, 53, 55, 56, 58, and 59

${ }^{\mathrm{c}}$ Northeast, West, and Midwest combined

dadjusted odds ratio

$* \mathrm{p}<0.05 ; * * \mathrm{p}<0.01 ; * * * \mathrm{p}<0.001$ 


\title{
Manuscript 2
}

\section{Impact of Virginia's HPV Vaccine School-Entry Mandate on HPV Vaccination among 13-17 Year-Old Females}

\begin{abstract}
The link between human papillomavirus (HPV) and anogenital cancers is well established in the literature. Many states have passed laws requiring funding for HPV education or vaccination. Mandatory HPV vaccination policies have been considered and passed in several states; yet their effectiveness has not been evaluated. This study sought to assess the impact of Virginia's HPV vaccine mandate for girls entering sixth grade on HPV vaccine uptake among adolescent females aged 13-17 years.

Data from the National Immunization Survey-Teen for the 2008-2012 period
\end{abstract} were used, and 3,203 adolescent females were included in the analysis. A difference-indifferences estimation, and logistic regression with a policy-period interaction term were performed. Virginia was considered the treatment state, and South Carolina and Tennessee were the comparison states to account for non-policy factors that may have affected vaccination rates during the time period considered in the analysis.

There was no evidence of an effect of the HPV vaccination policy on vaccination rates or on physician vaccination recommendation using either the difference-indifferences analysis or the policy-period interaction term in the logistic regression. Physician recommendation was the factor most strongly associated with vaccination in the Virginia-South Carolina analysis (aOR=10.3; 95\%CI: 6.4-16.6) and in the VirginiaTennessee analysis $(\mathrm{aOR}=9.33$; 95\%CI: 6.11-14.3).

Study findings suggest that Virginia's HPV vaccine mandate did not lead to a significant increase in HPV vaccination among adolescent females or physician 
recommendations. However, physician recommendation was strongly associated with vaccination.

\section{Background}

The link between human papillomavirus (HPV) and oropharyngeal, penile, anal, vulvar, vaginal, and cervical cancers is well established (Forman et al., 2012). To date, two HPV preventative vaccines have been licensed by the Food and Drug Administration (Centers for Disease Control and Prevention, 2010a, 2010b; U.S. Food and Drug Administration, 2014). The Advisory Committee on Immunization Practices (ACIP) recommends routine HPV immunization for 11-12 year-old adolescents (Centers for Disease Control and Prevention, 2012). HPV vaccination rates have increased in the United States, but they remain below Healthy People 2020's goal of $80 \%$ (United States Department of Health and Human Services, 2014). In 2014, among adolescent females aged 13-17 years, the overall HPV vaccine initiation rates were higher among nonHispanic Blacks (66.4\%) and Hispanics (66.3\%) as compared to non-Hispanic Whites (56.1\%) (Reagan-Steiner et al., 2015). However, HPV vaccine completion rates were very low for all ethnic groups, with Hispanics having the highest rates (46.9\%) followed by Blacks (39.0\%), Whites (37.5\%), and Asians (35.7\%) (Reagan-Steiner et al., 2015).

In January 2007, three bills (SB1230, HB2035, SB1914) were introduced in Virginia to include HPV vaccine among vaccines required for school (Virginia Legislative Information System, 2007). As originally introduced, SB1230 and HB2035 would require that females received three doses of the HPV vaccine (Virginia Legislative Information System, 2007). These bills did not include an opt-out option in addition to the regular exemptions for all other childhood vaccines (Virginia Legislative Information 
System, 2007). The third bill, SB1914, would require females to receive three doses of the HPV vaccine, but it provided an opt-out option to parents after having read approved HPV educational materials (Virginia Legislative Information System, 2007).

Before they were enacted, HB2035 and SB1230 incorporated SB 1914 based on the governor's recommendation to include an opt-out option to parents since HPV is not transmissible in a school setting (Virginia Legislative Information System, 2007). HB2035 and SB1230 are identical and were both effective on October 1, 2008 (Virginia Legislative Information System, 2007). In the District of Columbia, a bill requiring HPV vaccination certificate was introduced in January 2007 (Council of the District of Columbia, 2007). Before the bill was passed, similar to the change in Virginia's bills, it included an opt-out option for parents after having read the educational materials provided (Council of the District of Columbia, 2007).

More than twenty states have passed laws requiring funding for HPV education, or HPV vaccination while three others distribute free HPV vaccines through their health departments. Only the District of Columbia, Virginia, and Rhode Island have passed a HPV vaccine mandate (National Conference of States Legislatures, 2015). Mandatory school-entry HPV vaccine policy has been considered in several states; yet its impact on HPV vaccination has not been evaluated. This study assessed the impact of Virginia's HPV vaccine mandate for school-entry for girls entering sixth grade on vaccination among 13-17 year-old adolescent females, using data from the National Immunization Survey-Teen (NIS-Teen). 


\section{Methods}

\section{NIS-Teen Survey}

The purpose of the National Immunization Survey (NIS) is to estimate vaccination coverage among children 19 to 35 months old (Curtin, Lester et al., 2013). The NIS-Teen is a NIS appended survey, which uses random digit dialing telephone survey of households to provide an estimation of vaccination coverage among adolescents. NIS-Teen data collection is conducted in two stages: a household telephone survey that collects information on immunization status of adolescents from the teen's parent or guardian, along with a permission request to contact the adolescent's healthcare provider; and an immunization questionnaire mailed to teen's healthcare providers (Curtin, Lester et al., 2013). For the telephone interview, the adult who is most informed about the child's vaccinations is chosen to answer the questions. NIS-Teens' detailed survey methodologies have been published elsewhere (Curtin, Lester et al., 2013).

\section{Study Design}

The current study used a pre-post design (difference-in-differences) with a comparison group from a natural experiment. A natural experiment is one in which an intervention varies through the natural occurrence of an event that is exogenous to the outcome of interest (Petticrew et al., 2005; Remler \& Van Ryzin, 2011). It allows comparisons between a group that experiences the predetermined event and a group that did not (Petticrew et al., 2005; Remler \& Van Ryzin, 2011). Although several methods have been used to assess effectiveness of public health policies, difference-in-differences is among the most widely used methods (Mason et al., 2015; Rajaram et al., 2014). 
Difference-in-differences estimates the treatment effect on the treated group by subtracting the change in the outcome for the comparison group before and after the treatment from the change in the outcome for the treatment group before and after the treatment (Descy \& Tessaring, 2004; Meyer, 1995a). Taking the difference of the group differences allows the control of unobserved differences that may bias the treatment effect estimate (Descy \& Tessaring, 2004; Meyer, 1995a).

\section{Treatment and Comparison States}

Virginia is located in the Southern Black Belt, a part of the American South. Its neighboring states in the Black Belt include North Carolina, Tennessee, and South Carolina. North Carolina has enacted legislation requiring the Department of Health to provide HPV-related information to parents with children in grades five through 12 in 2007 (National Conference of States Legislatures, 2015). As a result, North Carolina could not be a comparison state for Virginia.

In South Carolina, a bill that would require HPV vaccine for girls after their $11^{\text {th }}$ birthday or before entering the sixth grade was introduced in 2007 . The bill was not enacted (National Conference of States Legislatures, 2015). Another bill that would require the Department of Health to offer HPV vaccine to girls before entering the seventh grade was introduced but was vetoed by the governor in June 2012. The House sustained the veto (National Conference of States Legislatures, 2015). HPV vaccine opponents expressed their concerns regarding side effects as well as potential increase in sexual promiscuity. On her side, Governor Haley stated lack of funding as her reason for vetoing the bill (South Carolina Radio Network, 2014). In Tennessee, mandatory HPV 
vaccine policy was not introduced. A bill that would require the Department of Health to provide a report of the prevalence of HPV infection by age group accompanied by a HPV vaccine recommendation was introduced but not enacted (National Conference of States Legislatures, 2015).

In the Black Belt, Virginia has the lowest poverty rate (10.4\%) (U.S. Census Bureau, 2014b), and highest high school graduation rate among 18-24 year-olds (87\%) for the 2007-2012 period compared to the other states in that region (U.S. Census Bureau, 2014a). Virginia is the only state in the region with median household income greater than the U.S. average. For the 2007-2010 period, Virginia's median household income was $\$ 60,503$ (U.S. Census Bureau, 2013a). For the same time period, the median household income was \$42,295 in South Carolina, and \$40,025 in Tennessee (U.S. Census Bureau, 2013a). During the 2007-2012 period, South Carolina had $82.3 \%$ of individuals aged 18-24 years with a high school diploma, and the poverty rate was $15.7 \%$ (U.S. Census Bureau, 2014a). During the same period, the poverty rate was $16.3 \%$, and 82.3\% of individuals aged 18-24 years had a high school diploma in Tennessee (U.S. Census Bureau, 2014a). Both South Carolina and Tennessee were chosen as comparison states for Virginia for a more robust analysis.

\section{Sample and Population}

Virginia's policy was implemented during the 2009-2010 school year (Virginia Department of Health, 2014). The pre-policy period was 2008 (before), the implementation year was 2009 (Virginia Department of Health, 2014), and the postpolicy period was 2010-2012 (after). The sample for this analysis included 1,064 female 
adolescents aged 13-17 years living in Virginia, 1,084 living in South Carolina, and 1,055 from Tennessee whose parent or guardian provided a response to HPV vaccination questions during the telephone survey.

\section{Variables}

Selected adolescent females' race/ethnicity, age, state of residence, health insurance status, healthcare visits in the past 12 months, HPV vaccination history, maternal education level, maternal income, and maternal marital status were included in the analysis. For the purpose of this study, teens who had received at least one dose of the HPV vaccine series were classified as vaccinated. Immunization data from healthcare providers tend to be more accurate than self-reported. However, adolescents with sufficient immunization information from their healthcare provider are a subset of those who participated in the telephone interview. The kappa agreement between HPV vaccination status from the telephone survey and HPV vaccination status from provider was $92.5 \%$.

\section{Statistical Analysis}

Weighted Pearson's chi-square test to investigate the variation of different demographic factors between Virginia and its comparison states was performed. Additionally, we estimated a difference-in-differences model to quantify the difference in the change in vaccination rates between Virginia and South Carolina, and between Virginia and Tennessee before and after the policy implementation. Subsequently, a logistic regression analysis with 'policy' and 'period' interaction was performed to estimate the difference-in-differences in HPV vaccination among females living in 
Virginia or its comparison states in order to control for demographic variables using the following model:

Logit $(\operatorname{Pr}(Y=1$ period, treatment, period*treatment, age..... physician recommendation $))$ $=\alpha+\Upsilon_{1} *$ state $+\Upsilon_{2} *$ period $+\Upsilon_{3} *($ period $*$ state $)+\boldsymbol{\beta} \boldsymbol{X}(1)$

$\mathrm{Y}$ is HPV vaccination $(1=$ yes $/ 0=$ no $)$, and $\boldsymbol{X}$ is a vector of control variables that included age, medical visits, maternal education, maternal income, and physician recommendation. Two logistic regression models were built to estimate difference-indifferences in HPV vaccination. The first model included the policy, the period, and the policy and period interaction variables. For the second model, the demographic variables were added to the first model. To estimate difference-in-differences in physician recommendation while controlling for demographic variables, two logistic regression models were built using the following model:

Logit $(\operatorname{Pr}(\mathrm{Y}=1$ period, treatment, period*treatment, age $\ldots . .$. and maternal income $))=\alpha+$ $\Upsilon_{1} *$ state $+\Upsilon_{2} *$ period $+\Upsilon_{3} *($ period $*$ state $)+\boldsymbol{\beta} \boldsymbol{X}(2)$

$\mathrm{Y}$ is HPV vaccine recommendation from a physician $(1=$ yes $/ 0=$ no $)$, and $\boldsymbol{X}$ is a vector of control variables. A linktest was performed to assess the fit of the model. A pvalue $<0.05$ was used as the threshold for statistical significance. All statistical analyses were performed using STATA 13 (StataCorp, 2013), and all estimates were weighted to females aged 13-17 in the relevant states and years included in each analysis. 


\section{Results}

\section{HPV Vaccination}

Vaccination rates followed similar trends in Virginia and South Carolina from 2008 to 2012 (Figure 1). In the pre-policy year, vaccination rates were 33.9\% in Virginia, $20.8 \%$ in South Carolina, and $27.0 \%$ in Tennessee. In 2009, which is the year of the implementation of Virginia's policy, vaccination rates were $37.9 \%$ in Virginia, $31.3 \%$ in South Carolina, and $40.7 \%$ in Tennessee. For the first year post-policy, vaccination rates were $47.1 \%, 39.7 \%$, and $29.7 \%$ in Virginia, South Carolina, and Tennessee, respectively. In 2011, the rates were $42.0 \%$ for Virginia, $34.7 \%$ in South Carolina, and $36.1 \%$ in Tennessee. The rates were $48.8 \%, 36.1 \%$, and $51.5 \%$ in Virginia, South Carolina, and Tennessee, respectively, in 2012 (Figure 1). The difference-in-differences in the vaccinated proportions between Virginia and South Carolina or Virginia and Tennessee was not significant (Table 1).

\section{[Figure 1 Here]}

\section{[Table 1 Here]}

For the difference in selected socio-demographic and health utilization characteristics, in the pre-policy period, the sample of adolescent females in Virginia and South Carolina differed by vaccination status, race/ethnicity, health insurance coverage history, and household income (Table 2). For Virginia and Tennessee, adolescents differed by race and ethnicity, age, health insurance coverage history, and household income (Table 2). In the post-policy period, the sample of adolescent females in Virginia and South Carolina differed by vaccination status, race/ethnicity, number of medical 
visits in the previous year, the proportion who had received a HPV vaccine recommendation, age at last medical visit, the proportion who had their 11-12-year-old medical check-up, maternal marital status, education, and income (Table 3). For Virginia and Tennessee, adolescents differed by vaccination status, race/ethnicity, mother's marital status, mother's education level, and income level (Table 3).

[Table 2 Here]

[Table 3 Here]

In the first Virginia-South Carolina logistic regression model, adolescent females in Virginia had greater odds of being vaccinated compared to those living in South Carolina (aOR=1.95; 95\%CI: 1.23-3.07). Females in both states had greater odds of vaccination in the post- compared to the pre-policy period $(\mathrm{aOR}=2.22 ; 95 \% \mathrm{CI}: 1.51-$ 3.27). The policy and period interaction term was not significant (Table 4). In the second Virginia-South Carolina model, adolescent females who were recommended the vaccine had higher odds of being vaccinated (aOR=10.3; 95\%CI: 6.4-16.6). Also, those who had four to six medical visits the previous year had higher odds of vaccination compared to those who had fewer visits $(\mathrm{aOR}=2.04 ; 95 \% \mathrm{CI}: 1.24-3.35)$ (Table 4).

In the first Virginia-Tennessee model, females were more likely to be vaccinated in the post- compared to the pre-policy period (aOR=1.71; 95\%CI: $1.19-2.44)$. The policy variable and the interaction term were not significant (Table 4). In the second Virginia-Tennessee model, females had higher odds of being vaccinated in the postcompared to the pre-policy period $(\mathrm{aOR}=2.26$; 95\%CI: $1.02-5.00)$. Females who were 
recommended the vaccine had higher odds of being vaccinated $(\mathrm{aOR}=9.33$; 95\%CI: $6.11-$ 14.3) (Table 4).

[Table 4 Here]

\section{Physician Recommendation}

Physician recommendation rates followed similar trends in Virginia and South Carolina from 2008 to 2012 (Figure 2). In 2008, the pre-policy year, vaccine recommendation rates were $46.7 \%$ in Virginia, $41.2 \%$ in South Carolina, and $40.9 \%$ in Tennessee. In 2009, the year of the implementation of Virginia's policy, vaccine recommendation rates were 52.6\% in Virginia, $41.6 \%$ in South Carolina, and $48.5 \%$ in Tennessee. For the first post-policy year, vaccine recommendation rates were $51.2 \%$, 44.7\%, and 49.5\% in Virginia, South Carolina, and Tennessee, respectively. In 2011, the rates were 55.6\% in Virginia, $48.0 \%$ in South Carolina, and $49.1 \%$ in Tennessee. The rates were $65.4 \%, 50.8 \%$, and $66.5 \%$ in Virginia, South Carolina, and Tennessee, respectively, in 2012 (Figure 2).

\section{[Figure 2 Here]}

The difference-in-differences in the percentage of female adolescents who received physician recommendation for HPV vaccine between Virginia and South Carolina and between Virginia and Tennessee was not significant (Table 5). For the first Virginia-South Carolina logistic regression model predicting the odds for physician recommendation, none of the variables was significant. For the second model, females who had seven to nine medical visits had higher odds to receive a physician recommendation for HPV vaccine compared to those who had fewer visits $(\mathrm{aOR}=2.36$; 
95\%CI: 1.09-5.11). Those whose family income was unknown had lower odds of receiving a $\mathrm{HPV}$ vaccine recommendation $(\mathrm{aOR}=0.33 ; 95 \% \mathrm{CI}$ : $0.14-0.76)$. Female adolescents who were continually insured since age 11 had lower odds of receiving a HPV vaccine recommendation $(\mathrm{aOR}=0.33$; 95\%CI: 0.17-0.66) (Table 6).

In the first Virginia-Tennessee model, the odds of HPV vaccine recommendation were higher in the post- compared to the pre-policy period $(\mathrm{aOR}=1.77$; $95 \% \mathrm{CI}: 1.28-$ 2.45). The remaining variables were not significant (Table 6). In the second model, females whose mother graduated high school (aOR=2.24; 95\%CI: 1.09-4.6), had some college education $(\mathrm{aOR}=3.14 ; 95 \% \mathrm{CI}$ : $1.49-6.59)$, or graduated college $(\mathrm{aOR}=2.87$; 95\%CI: 1.34-6.1) had higher odds of receiving a physician recommendation for the vaccine compared to those whose mother did not graduate from high school.

Additionally, females living in household with income level above poverty and $\leq \$ 75,000$ had lower odds of receiving a HPV vaccine recommendation compared to those in households of more than $\$ 75,000$ (Table 6).

[Table 5 Here]

[Table 6 Here]

\section{Discussion}

There was no evidence of improvement in vaccination rates associated with the mandatory school-entry vaccination policy in either the Virginia-Tennessee or the Virginia-South Carolina comparisons. Results were similar in both the difference-indifferences analysis and the logistic regression. Moreover, after we controlled for demographic factors, females in Virginia did not have higher odds of being vaccinated 
compared to those either in South Carolina or Tennessee. Those who had more than three medical visits in the previous year had higher odds of being vaccinated. Females from all three states had higher odds of vaccination in the post- compared to the pre-policy period. HPV vaccine recommendation was consistently associated with HPV vaccination. This association is in agreement with the literature (Mazza, Petrovic, \& Chakraborty, 2012; Perkins et al., 2013; Reiter, McRee, et al., 2013; Vadaparampil et al., 2011). However, there was also no evidence that the mandatory vaccination for school-entry increased physician recommendations for the vaccine.

There are several reasons why Virginia's HPV vaccine school-entry mandate may not have resulted in a significant increase in HPV vaccination. First, parents or guardians of females entering sixth grade were not required to provide proof of HPV immunization like they do for other required vaccines (Virginia Department of Health, 2014). Second, after reading the HPV educational materials, parents who opposed HPV vaccination for their daughters could opt-out without having to provide any documentation for their refusal. Additionally, parents in Virginia have not only expressed concerns regarding the safety of the vaccine (Liddon, Hood, \& Leichliter, 2012), but also about the possibility that adolescents will interpret their receipt of the vaccine as a license to be sexually active or to practice risky sexual behaviors (Scarinci, Garcés-Palacio, \& Partridge, 2007;

Schuler, Reiter, Smith, Brewer, \& Hill, 2011). Moreover, negative opinions regarding the HPV vaccine mandate were common in the media (Casciotti et al., 2014), and several parental rights groups viewed the mandate as an infringement on parental rights despite the loose opt-out option (Casciotti et al., 2014; Natural News Network, 2015; PR Newswire, 2015). Reasons cited for mandate opposition included government distrust, 
sexual transmission concern, and infringement of parental autonomy (Casciotti et al., 2014). Furthermore, since the passing of the mandate, the legislature has introduced several bills to repeal it (National Conference of States Legislatures, 2015). Evidently, Virginia's socio-political environment did not facilitate the effectiveness of the mandate.

\section{Strengths and Limitations}

Although this study is among the firsts to assess the impact of Virginia's mandate on HPV vaccination, it is not without limitations. One major limitation of the research is the use of pooled cross-sectional data. Moreover, the policy and the control states have some similarities but are not exactly identical. Difference-in-differences assumes that time-varying unmeasured characteristics are constant over the time period in the policy state and do not correlate with HPV vaccinations. Although we verified that any significant change did not take place for some of the unmeasured characteristics in the dataset such as bills directly impacting Medicaid expansion under the Affordable Care Act and Medicaid and CHIP eligibility. This assumption could not be fully tested.

Despite the aforementioned limitations, the present study used data from a nationally representative sample that is robust against selection bias. Additionally, this study is among the first few to assess the impact of Virginia's HPV vaccine mandate for school-entry on vaccine uptake among female adolescents while similar policies are being considered in several states. Moreover, the initial sample size decreased for the logistic regression models due to missing observations, but the power did not fall below $80 \%$. Furthermore, a general pitfall in policy analysis is "policy endogeneity," which would occur, in this case, if Virginia's HPV vaccine school-entry mandate were adopted 
to increase HPV vaccination, because of low HPV vaccination in Virginia. There was no indication that Virginia adopted the policy mandate due to low HPV vaccination. Prior to the passing of the mandate, Virginia's HPV vaccination rates were higher than those of the neighboring states. Therefore, there is no evidence of "policy endogeneity".

\section{Implications for Practice}

The current study assessed an important policy that may have served as a model for other states with high cervical cancer rates. Results revealed that the HPV vaccine mandate for school-entry did not yield the intended results. However, these results do not suggest that HPV vaccine mandates cannot be successful. The socio-political environment in which the mandate was passed is an important factor that may influence its impact. In Virginia, the socio-political context was not favorable to the mandate. Most parents had a positive perception of physician recommendation even if they chose not to vaccinate their adolescents (Perkins et al., 2014). Therefore, it is necessary for policymakers to understand that physician recommendation must accompany the mandate in order for it to yield the desired results. Since the introduction of HPV vaccine, the prevalence of HPV infection has decreased by more than 50\% among 14-19 year-old females in the United States (Markowitz et al., 2013), despite low HPV vaccination rates. We must continue to educate parents about the vaccine's effectiveness at reducing HPV infection from the types covered by the vaccine and the implications of this decline in order to facilitate physician recommendation. 


\section{Conclusion}

Study findings did not indicate that Virginia's HPV vaccine mandate led to a significant increase in HPV vaccination among adolescent females. Despite Virginia's mandate, physician recommendation remains the consistent predictor of HPV vaccination. While the mandate may be viewed as infringement of parental rights by some, a physician recommendation is not viewed as such. As a result, efforts to encourage physician recommendation must continue along with research to better understand the facilitators of physician recommendation.

\section{References}

Casciotti, D. M., Smith, K. C., Andon, L., Vernick, J., Tsui, A., \& Klassen, A. C. (2014). Print news coverage of school-based human papillomavirus vaccine mandates. Journal of School Health, 84(2), 71-81. doi:10.1111/josh.12126

Centers for Disease Control and Prevention. (2010a). FDA licensure of bivalent human papillomirus vaccine (HPV2, Cervarix) for use in females and updated HPV vaccination recommendations from the Advisory Committee on Immunization Practices (ACIP). MMWR, 59(20), 625-629.

Centers for Disease Control and Prevention. (2010b). FDA licensure of quadrivalent human papillomavirus vaccine (HPV4, Gardasil) for use in males and guidance from the Advisory Committee on Immunization Practices (ACIP). MMWR, 56(20), 630 632.

Centers for Disease Control and Prevention. (2012). Advisory Committee on Immunization Practices: human papillomavirus vaccine resolution. Retrieved March 13, 2015, from http://www.cdc.gov/vaccines/programs/vfc/providers/resolutions.html

Council of the District of Columbia. (2007). Human Papillomavirus vaccination and reporting Act of 2007. Retrieved February 26, 2016, from http://lims.dccouncil.us/Legislation/B17-0030?FromSearchResults=true

Curtin, Lester, R., Mohadjer, L. K., Dohrmann, S. M., Kruszan-Moran, D., Mirel, L. B., Carroll, M. D., ... Johnson, C. L. (2013). National Health and Nutrition Examination Survey: Sample design, 2007 - 2010. Vital and Health Statistics, 2(160). 
Descy, P., \& Tessaring, M. (2004). Evaluation and impact of education and training: the value of learning. Retrieved from http://www.trainingvillage.gr/etv/projects_Networks/researchlab/

Forman, D., de Martel, C., Lacey, C. J., Soerjomataram, I., Lortet-Tieulent, J., Bruni, L., ... Franceschi, S. (2012). Global burden of human papillomavirus and related diseases. Vaccine, 30(Suppl 5). doi:10.1016/j.vaccine.2012.07.055

Liddon, N. C., Hood, J. E., \& Leichliter, J. S. (2012). Intent to receive HPV vaccine and reasons for not vaccinating among unvaccinated adolescent and young women: findings from the 2006-2008 National Survey of Family Growth. Vaccine, 30(16), 2676-82. doi:10.1016/j.vaccine.2012.02.007

Markowitz, L. E., Hariri, S., Lin, C., Dunne, E. F., Steinau, M., McQuillan, G., \& Unger, E. R. (2013). Reduction in human papillomavirus (HPV) prevalence among young women following HPV vaccine introduction in the United States, National Health and Nutrition Examination Surveys, 2003-2010. The Journal of Infectious Diseases, 208(3), 385-93. doi:10.1093/infdis/jit192

Mason, T., Sutton, M., Whittaker, W., McSweeney, T., Millar, T., Donmall, M., ... Pierce, M. (2015). The impact of paying treatment providers for outcomes: Difference-in-differences analysis of the "payment by results for drugs recovery" pilot. Addiction, 110(7), 1120-8. doi:10.1111/add.12920

Mazza, D., Petrovic, K., \& Chakraborty, S. (2012). HPV vaccination of adult women: an audit of Australian general practitioners. The Australian \& New Zealand Journal of Obstetrics \& Gynaecology, 52(6), 528-33. doi:10.1111/ajo.12002

Meyer, B. D. (1995). Natural and Quasi-Experiments in Economics. Journal of Business \& Economic Statistics, 13(2), 151. doi:10.2307/1392369

National Conference of States Legislatures. (2015). HPV Vaccine: State Legislation and Statutes. Retrieved December 24, 2015, from

http://www.ncsl.org/research/health/hpv-vaccine-state-legislation-and-statutes.aspx

Natural News Network. (2015). Virginia law guaranteeing parents' medical rights routed by mandatory HPV vaccination - NaturalNews.com. Retrieved December 23, 2015, from http://www.naturalnews.com/021628_HPV_vaccine_Merck.html

Perkins, R. B., Apte, G., Marquez, C., Porter, C., Belizaire, M., Clark, J. A., \& Pierrejoseph, N. (2013). Factors Affecting Human Papillomavirus Vaccine Use Among White, Black and Latino Parents of Sons. The Pediatric Infectious Disease Journal, 32(1), 38-44. doi:10.1097/INF.0b013e31826f53e3 
Perkins, R. B., Clark, J. a, Apte, G., Vercruysse, J. L., Sumner, J. J., Wall-Haas, C. L., ... Pierre-Joseph, N. (2014). Missed opportunities for HPV vaccination in adolescent girls: a qualitative study. Pediatrics, 134(3), e666-74. doi:10.1542/peds.2014-0442

Petticrew, M., Cummins, S., Ferrell, C., Findlay, A., Higgins, C., Hoy, C., ... Sparks, L. (2005). Natural experiments: an underused tool for public health? Public Health, 119(9), 751-7. doi:10.1016/j.puhe.2004.11.008

PR Newswire. (2015). Virginia Committee Ignores Parental Rights, Health Concerns of HPV Vaccine Mandate. Retrieved December 23, 2015, from http://www.prnewswire.com/news-releases/virginia-committee-ignores-parentalrights-health-concerns-of-hpv-vaccine-mandate-57101112.html?\$G1Ref

Rajaram, R., Chung, J. W., Jones, A. T., Cohen, M. E., Dahlke, A. R., Ko, C. Y., ... Bilimoria, K. Y. (2014). Association of the 2011 ACGME resident duty hour reform with general surgery patient outcomes and with resident examination performance. JAMA, 312(22), 2374-2384. doi:10.1001/jama.2014.15277

Reagan-Steiner, S., Yankey, D., Jeyarajah, J., Elam-Evans, L. D., Singleton, J. A., Curtis, C. R., ... Stokley, S. (2015). National, Regional, State, and Selected Local Area Vaccination Coverage among Adolescents Aged 13-17--United States, 2014. $M M W R, 64(29), 784-792$. Retrieved from http://www.ncbi.nlm.nih.gov/pubmed/26225479

Reiter, P. L., McRee, A.-L., Pepper, J. K., Gilkey, M. B., Galbraith, K. V, \& Brewer, N. T. (2013). Longitudinal predictors of human papillomavirus vaccination among a national sample of adolescent males. American Journal of Public Health, 103(8), 1419-27. doi:10.2105/AJPH.2012.301189

Remler, D. K., \& Van Ryzin, G. G. (2011). Natural and quasi experiments. In Research methods in practice: Strategies for description and causation (pp. 427-464).

Thousand Oaks: California: Sage.

Scarinci, I. C., Garcés-Palacio, I. C., \& Partridge, E. E. (2007). An examination of acceptability of HPV vaccination among African American women and Latina immigrants. Journal of Women's Health, 16(8), 1224-33. doi:10.1089/jwh.2006.0175

Schuler, C. L., Reiter, P. L., Smith, J. S., Brewer, T., \& Hill, C. (2011). HPV vaccine and behavioral disinhibition. Sexually Transmitted Infections, 87(4), 349-353.

doi:10.1136/sti.2010.048017.HPV

South Carolina Radio Network. (2014). CDC: South Carolina among most improved fro HPV vaccine availability. Retrieved March 11, 2016, from http://www.southcarolinaradionetwork.com/?s=HPV+vaccine 
StataCorp. (2013). Stata Statistical Software: Release 13. College Station, Texas: StataCorp LP.

U.S. Census Bureau. (2013). Income. Retrieved April 27, 2015, from http://www.census.gov/hhes/www/income/

U.S. Census Bureau. (2014a). Educational attainment. Retrieved from httos://www.census.gov/hhes/socdemo/education/

U.S. Census Bureau. (2014b). Poverty. Retrieved April 27, 2015, from http://www.census.gov/hhes/www/poverty/

U.S. Food and Drug Administration. (2014). Press Announcements - FDA approves Gardasil 9 for prevention of certain cancers caused by five additional types of HPV. Retrieved June 18, 2015, from http://www.fda.gov/NewsEvents/Newsroom/PressAnnouncements/ucm426485.htm

United States Department of Health and Human Services. (2014). Healthy People 2020 topics and objectives:Immunization and infectious diseases. Retrieved March 5, 2016, from https://www.healthypeople.gov/2020/topicsobjectives/topic/immunization-and-infectious-diseases

Vadaparampil, S. T., Kahn, J. A., Salmon, D., Lee, J.-H., Quinn, G. P., Roetzheim, R., ... Giuliano, A. R. (2011). Missed clinical opportunities: provider recommendations for HPV vaccination for 11-12 year old girls are limited. Vaccine, 29(47), 8634-41. doi:10.1016/j.vaccine.2011.09.006

Virginia Department of Health. (2014). School and Day Care Minimum Immunization Requirements. Retrieved December 24, 2015, from http://www.vdh.virginia.gov/epidemiology/immunization/requirements.htm

Virginia Legislative Information System. (2007). Bill Tracking - 2007 Session Legislation. Retrieved February 26, 2016, from http://leg1.state.va.us/cgibin/legp504.exe?071+ful+CHAP0858 
Figure 1. HPV vaccination't trends in Virginia, South Carolina, and Tennessee, 20082012

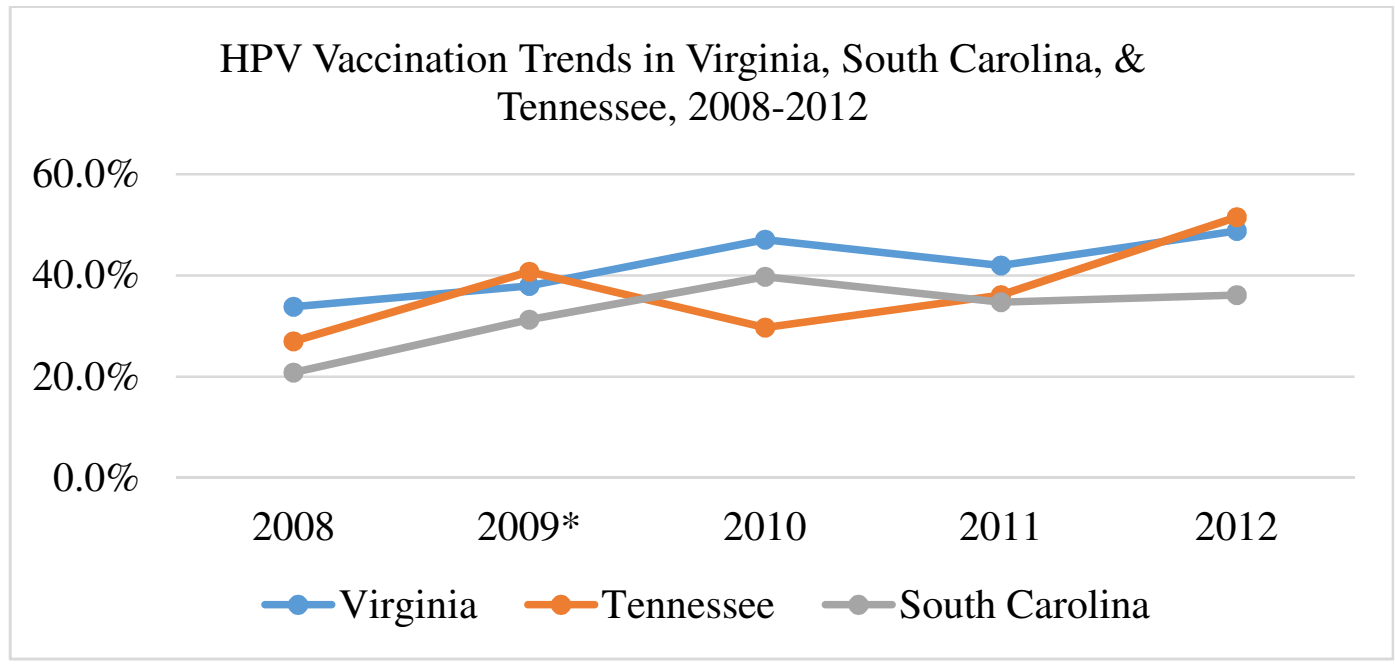

*Policy implementation year

$\geq 1$ dose of HPV vaccine

Table 1. Difference-in-differences in HPV vaccination ${ }^{\dagger}$ rates, Virginia and South Carolina, and Virginia and Tennessee

\begin{tabular}{lccc}
\hline \multicolumn{1}{c}{ N=2,119 } & $\begin{array}{c}\text { Pre-policy Period } \\
\mathbf{( 2 0 0 8 )}\end{array}$ & $\begin{array}{c}\text { Post-policy period } \\
\mathbf{( 2 0 1 0 - 1 2 )}\end{array}$ & Difference \\
\hline Virginia & 0.339 & 0.460 & 0.121 \\
\hline South Carolina & 0.208 & 0.368 & 0.160 \\
\hline Difference-in-differences & & & -0.039 \\
\hline \multicolumn{1}{c}{$\mathbf{N = \mathbf { 2 , 1 3 9 }}$} & & & \\
\hline Virginia & 0.339 & 0.460 & 0.121 \\
\hline Tennessee & 0.27 & 0.387 & 0.117 \\
\hline Difference-in-differences & & & 0.004 \\
$* \mathrm{p}<0.05$ & & & \\
$\mathrm{1} \geq 1$ dose of HPV vaccine & & &
\end{tabular}


Table 2. Difference in selected demographic characteristics in Virginia and South Carolina, and Virginia and Tennessee pre-policy period

\begin{tabular}{|c|c|c|c|c|c|}
\hline \multirow{3}{*}{ Characteristics } & \multicolumn{5}{|c|}{ Pre-policy period (2008) } \\
\hline & $\begin{array}{l}\text { Virginia } \\
(\mathbf{n}=\mathbf{2 9 8})\end{array}$ & $\begin{array}{l}\text { S. Carolina } \\
(\mathbf{n}=\mathbf{2 9 5})\end{array}$ & $\begin{array}{l}\mathrm{p}- \\
\text { value }\end{array}$ & $\begin{array}{c}\text { Tennessee } \\
(\mathbf{n}=239)\end{array}$ & $\begin{array}{c}\mathrm{p}- \\
\text { value }\end{array}$ \\
\hline & $\%(95 \% \mathrm{CI})$ & $\%(95 \% \mathrm{CI})$ & & $\%(95 \% \mathrm{CI})$ & \\
\hline Vaccinated & & & 0.004 & & 0.14 \\
\hline No & $\begin{array}{l}66.2(59.0- \\
72.6)\end{array}$ & $79.2(73.0-84.3)$ & & $\begin{array}{l}73.0(66.5- \\
78.6)\end{array}$ & \\
\hline Yes & $33.8(27-41)$ & $20.8(15.8-27.0)$ & & $\begin{array}{l}27.0(21.3- \\
33.5)\end{array}$ & \\
\hline Race and ethnicity & & & 0.04 & & 0.042 \\
\hline Hispanic & $6.9(4.2-11.3)$ & $4.7(2.4-8.5)$ & & $3.2(1.5-6.9)$ & \\
\hline White & $\begin{array}{l}64.7(57.2- \\
71.6)\end{array}$ & $55.4(48.7-61.8)$ & & $\begin{array}{l}70.9(63.6- \\
77.3)\end{array}$ & \\
\hline Black & $\begin{array}{l}24.4(18.0- \\
32.2)\end{array}$ & $36.8(30.5-43.7)$ & & $\begin{array}{c}22.3(16.3- \\
29.8)\end{array}$ & \\
\hline Other & $3.9(2.2-6.7)$ & $3.2(1.8-5.7)$ & & $3.6(1.9-6.5)$ & \\
\hline Teen's age & & & 0.47 & & 0.048 \\
\hline 13-15years & $\begin{array}{l}64.7(57.6- \\
71.3)\end{array}$ & $61.3(54.8-67.4)$ & & $\begin{array}{c}55.0(48.3- \\
61.6)\end{array}$ & \\
\hline 16-17 years & $\begin{array}{c}35.3(28.7- \\
42.4)\end{array}$ & $38.7(32.6-45.2)$ & & $\begin{array}{l}45.0(38.4- \\
51.7)\end{array}$ & \\
\hline $\begin{array}{l}\text { No. of medical } \\
\text { visits last year }\end{array}$ & & & 0.57 & & 0.36 \\
\hline 1 to 3 & $\begin{array}{l}72.8(66.1- \\
78.6)\end{array}$ & 77.1(70.8-82.4) & & $\begin{array}{l}75.5(69.3- \\
80.8)\end{array}$ & \\
\hline 4 to 6 & $\begin{array}{l}22.7(17.3- \\
29.1)\end{array}$ & $19.6(14.7-25.7)$ & & $\begin{array}{l}17.9(13.2- \\
23.9)\end{array}$ & \\
\hline 7 to 9 & $4.6(2.4-8.5)$ & $3.3(1.5-6.9)$ & & $6.6(4.3-9.9)$ & \\
\hline $\begin{array}{l}\text { Ever been insured } \\
\text { since age } 11 ?\end{array}$ & & & 0.049 & & 0.009 \\
\hline yes & $4.0(1.8-8.5)$ & $9.4(6.1-14.2)$ & & $12.2(7.8-18.6)$ & \\
\hline No & $\begin{array}{l}96.0(91.5- \\
98.2)\end{array}$ & $90.6(85.8-93.9)$ & & $\begin{array}{l}87.8(81.4- \\
92.2)\end{array}$ & \\
\hline $\begin{array}{l}\text { HPV vaccine } \\
\text { recommendation }\end{array}$ & & & 0.27 & & 0.24 \\
\hline No & $\begin{array}{l}53.3(45.9- \\
60.5)\end{array}$ & $58.8(52.0-65.3)$ & & $\begin{array}{l}59.1(52.3- \\
65.5)\end{array}$ & \\
\hline Yes & $\begin{array}{l}46.7(39.5- \\
54.1)\end{array}$ & $41.2(34.7-48.0)$ & & $\begin{array}{c}40.9(34.5- \\
47.7)\end{array}$ & \\
\hline
\end{tabular}




\begin{tabular}{|c|c|c|c|c|c|}
\hline $\begin{array}{l}\text { Age at last medical } \\
\text { visits }\end{array}$ & & & 0.63 & & \\
\hline less than 11 years & $4.9(2.4-9.6)$ & $6.0(3.7-9.6)$ & & $4.7(2.7-8.2)$ & 0.94 \\
\hline 11 years or older & $\begin{array}{l}\text { 95.1(90.4- } \\
97.6)\end{array}$ & $94.0(90.4-96.4)$ & & $\begin{array}{l}95.3(91.8- \\
97.3)\end{array}$ & \\
\hline $\begin{array}{l}\text { Teen had an 11-12- } \\
\text { year-old check-up? }\end{array}$ & & & 0.09 & & \\
\hline No & $7.6(4.0-13.7)$ & $13.7(9.5-19.3)$ & & $9.6(6.1-14.7)$ & 0.53 \\
\hline Yes & $\begin{array}{l}92.4(86.3- \\
96.0)\end{array}$ & $86.3(80.7-90.5)$ & & $\begin{array}{l}90.4(85-3- \\
93.9)\end{array}$ & \\
\hline $\begin{array}{l}\text { Marital status of } \\
\text { mother }\end{array}$ & & & 0.49 & & 0.23 \\
\hline Married & $\begin{array}{l}54.2(38.0- \\
69.6)\end{array}$ & $61.2(49.2-72.1)$ & & $\begin{array}{l}\text { 67.3(51.7- } \\
79.8)\end{array}$ & \\
\hline Not married & $\begin{array}{l}45.8(30.4- \\
43.6)\end{array}$ & $88.8(27.9-50.8)$ & & $\begin{array}{l}32.7(20.2- \\
48.3)\end{array}$ & \\
\hline $\begin{array}{l}\text { Mother's education } \\
\text { level }\end{array}$ & & & 0.13 & & 0.33 \\
\hline $\begin{array}{r}\text { Less than high } \\
\text { school }\end{array}$ & $9.5(6.0-14.8)$ & $11.5(8.1-16.2)$ & & $12.8(8.6-18.6)$ & \\
\hline High school & $\begin{array}{l}27.4(21.1- \\
34.8)\end{array}$ & $31.6(25.4-38.5)$ & & $\begin{array}{l}32.9(26.6- \\
40.0)\end{array}$ & \\
\hline Some College & $\begin{array}{l}25.7(20.0- \\
32.3)\end{array}$ & $30.6(25.1-36.8)$ & & $\begin{array}{l}24.0(18.9- \\
29.8)\end{array}$ & \\
\hline college graduate & $\begin{array}{l}37.4(31.0- \\
44.3)\end{array}$ & 26.2(21.4-31.7) & & $\begin{array}{l}30.3(25.4- \\
\quad 36.2)\end{array}$ & \\
\hline Household income & & & 0.005 & & $\begin{array}{c}0.001 \\
4\end{array}$ \\
\hline $\begin{array}{r}\text { Above poverty } \\
>\$ 75,000\end{array}$ & $\begin{array}{l}47.0(40.0- \\
54.2)\end{array}$ & 29.7(24.3-35.7) & & $\begin{array}{l}29.4(24.2- \\
35.2)\end{array}$ & \\
\hline $\begin{array}{r}\text { Above poverty } \\
<=\$ 75,000\end{array}$ & $\begin{array}{l}42.6(35.5- \\
49.9)\end{array}$ & $\begin{array}{l}48.5(42.0- \\
54.98)\end{array}$ & & $\begin{array}{l}51.2(44.6- \\
57.8)\end{array}$ & \\
\hline $\begin{array}{r}\text { Below poverty } \\
\text { level }\end{array}$ & $7.2(4.3-11.8)$ & $16.6(12.1-22.2)$ & & $14.0(9.5-20.2)$ & \\
\hline Unknown & $3.2(1.5-6.6)$ & $5.2(3.2-8.5)$ & & $5.4(3.2-8.9)$ & \\
\hline
\end{tabular}


Table 3. Difference in selected demographic characteristics in Virginia and South Carolina, and Virginia and Tennessee post-policy period

\begin{tabular}{|c|c|c|c|c|c|}
\hline \multirow{3}{*}{ Characteristics } & \multicolumn{5}{|c|}{ Post-policy period (2010-12) } \\
\hline & $\begin{array}{l}\text { Virginia } \\
(\mathbf{n}=766)\end{array}$ & $\begin{array}{l}\text { S. Carolina } \\
(n=789)\end{array}$ & $\begin{array}{c}\mathrm{p}- \\
\text { value }\end{array}$ & $\begin{array}{c}\text { Tennessee } \\
(\mathbf{n}=816)\end{array}$ & $\begin{array}{l}\text { p- } \\
\text { value }\end{array}$ \\
\hline & $\%(95 \% \mathrm{CI})$ & $\%(95 \% \mathrm{CI})$ & & $\%(95 \% \mathrm{CI})$ & \\
\hline Vaccinated & & & 0.004 & & 0.023 \\
\hline No & $\begin{array}{l}54.0(49.6- \\
58.5)\end{array}$ & $63.2(58.7-67.4)$ & & $\begin{array}{c}61.3(56.9- \\
65.5)\end{array}$ & \\
\hline Yes & $\begin{array}{l}46.0(41.5- \\
50.4)\end{array}$ & $36.8(32.6-41.3)$ & & $\begin{array}{c}38.7(34.5- \\
43.1)\end{array}$ & \\
\hline Race and ethnicity & & & $\begin{array}{l}7 \mathrm{E}- \\
04\end{array}$ & & 0.003 \\
\hline Hispanic & $\begin{array}{l}8.6(6.2- \\
11.8)\end{array}$ & $4.4(3.0-6.4)$ & & $4.5(3.0-6.5)$ & \\
\hline White & $\begin{array}{c}59.7(55.2- \\
64.0)\end{array}$ & $56.6(52.0-61.0)$ & & $\begin{array}{l}70.2(65.9- \\
74.3)\end{array}$ & \\
\hline Black & $\begin{array}{l}24.1(20.1- \\
28.7)\end{array}$ & $34.0(29.6-38.7)$ & & $\begin{array}{l}20.3(16.6- \\
24.6)\end{array}$ & \\
\hline Other & $7.6(5.9-9.6)$ & $5.0(3.3-7.6)$ & & $5.0(3.4-7.2)$ & \\
\hline Teen's age & & & 0.41 & & 0.7 \\
\hline 13-15years & $\begin{array}{c}61.4(57.2- \\
65.4)\end{array}$ & $63.8(59.0-67.9)$ & & $\begin{array}{l}60.2(60.0- \\
64.3)\end{array}$ & \\
\hline $16-17$ years & $\begin{array}{c}38.6(34.6- \\
42.8)\end{array}$ & $36.2(32.1-40.5)$ & & $\begin{array}{c}39.8(35.7- \\
44.0)\end{array}$ & \\
\hline $\begin{array}{l}\text { No. of medical } \\
\text { visits last year }\end{array}$ & & & 0.026 & & 0.16 \\
\hline 1 to 3 & $\begin{array}{c}79.8(76.2- \\
82.9)\end{array}$ & $73.2(69.0-77.0)$ & & $\begin{array}{c}78.0(74.3- \\
81.3)\end{array}$ & \\
\hline 4 to 6 & $\begin{array}{c}16.6(13.7- \\
19.9)\end{array}$ & $20.5(17.0-24.4)$ & & $\begin{array}{l}15.8(13.0- \\
19.2)\end{array}$ & \\
\hline 7 to 9 & $3.7(2.4-5.6)$ & $6.3(4.5-8.8)$ & & $6.1(4.4-8.4)$ & \\
\hline $\begin{array}{l}\text { Ever been insured } \\
\text { since age } 11 ?\end{array}$ & & & 0.09 & & 0.74 \\
\hline yes & $\begin{array}{c}7.1(4.5- \\
11.0)\end{array}$ & $11.6(8.2-16.2)$ & & $7.9(5.2-11.6)$ & \\
\hline No & $\begin{array}{l}92.9(89.0- \\
95.5)\end{array}$ & $88.4(83.8-91.8)$ & & $\begin{array}{c}92.1(89.4- \\
94.8)\end{array}$ & \\
\hline $\begin{array}{l}\text { HPV vaccine } \\
\text { recommendation }\end{array}$ & & & 0.003 & & 0.46 \\
\hline No & $\begin{array}{c}42.6(38.3- \\
47.0)\end{array}$ & $52.2(47.7-56.6)$ & & $\begin{array}{c}44.9(40.6- \\
49.3)\end{array}$ & \\
\hline
\end{tabular}




\begin{tabular}{|c|c|c|c|c|c|}
\hline Yes & $\begin{array}{c}57.4 \\
(53.61 .7)\end{array}$ & $47.8(43.4-52.3)$ & & $\begin{array}{l}55.1(50.7- \\
59.4)\end{array}$ & \\
\hline $\begin{array}{l}\text { Age at last medical } \\
\text { visits }\end{array}$ & & & 0.004 & & 0.14 \\
\hline less than 11 years & $2.4(1.5-3.8)$ & $5.8(3.8-8.6)$ & & $3.9(2.5-6.1)$ & \\
\hline 11 years or older & $\begin{array}{l}97.6(96.2- \\
98.5)\end{array}$ & $\begin{array}{l}94.2(91.4- \\
96.2)\end{array}$ & & $\begin{array}{l}96.1(93.9- \\
97.5)\end{array}$ & \\
\hline $\begin{array}{l}\text { Teen had an 11-12 } \\
\text { year-old check up? }\end{array}$ & & & 0.032 & & 0.34 \\
\hline No & $6.7(4.7-9.4)$ & $10.8(8.2-14.3)$ & & $\begin{array}{l}91.7(89.1- \\
93.7)\end{array}$ & \\
\hline Yes & $\begin{array}{l}93.3(90.6- \\
95.3)\end{array}$ & $89.2(85.7-91.8)$ & & $8.3(6.3-10.9)$ & \\
\hline $\begin{array}{l}\text { Marital status of } \\
\text { mother }\end{array}$ & & & $\begin{array}{l}\text { 1E- } \\
05\end{array}$ & & 0.001 \\
\hline Married & $\begin{array}{l}74.7(70.4- \\
78.6)\end{array}$ & $56.9(52.3-61.4)$ & & $\begin{array}{c}64.8(60.4- \\
69.0)\end{array}$ & \\
\hline Not married & $\begin{array}{l}25.3(21.4- \\
29.7)\end{array}$ & $43.1(38.6-47.7)$ & & $\begin{array}{c}35.2(31.0- \\
39.6)\end{array}$ & \\
\hline $\begin{array}{l}\text { Mother's education } \\
\text { level }\end{array}$ & & & $\begin{array}{c}1 \mathrm{E}- \\
05\end{array}$ & & 0.002 \\
\hline $\begin{array}{r}\text { Less than high } \\
\text { school }\end{array}$ & $\begin{array}{l}7.6(5.5- \\
10.3)\end{array}$ & 11.1(8.4-14.6) & & $9.9(7.6-12.9)$ & \\
\hline High school & $\begin{array}{l}23.4(19.6- \\
27.7)\end{array}$ & $30.0(25.9-34.5)$ & & $\begin{array}{c}29.9(26.0- \\
34.0)\end{array}$ & \\
\hline Some College & $\begin{array}{l}23.6(20.1- \\
27.5)\end{array}$ & $29.8(25.9-33.9)$ & & $\begin{array}{l}26.8(23.1- \\
30.8)\end{array}$ & \\
\hline college graduate & $\begin{array}{l}45.4(41.3- \\
49.7)\end{array}$ & $29.1(25.7-32.7)$ & & $\begin{array}{l}33.4(29.7- \\
37.5)\end{array}$ & \\
\hline Household income & & & $\begin{array}{c}1 \mathrm{E}- \\
05\end{array}$ & & $\begin{array}{l}1 E- \\
05\end{array}$ \\
\hline $\begin{array}{r}\text { Above poverty } \\
>\$ 75,000 \\
\end{array}$ & $\begin{array}{c}44.0(39.9- \\
48.1)\end{array}$ & $25.0(22.0-28.3)$ & & $\begin{array}{l}27.1(23.8- \\
30.6)\end{array}$ & \\
\hline $\begin{array}{r}\text { Above poverty } \\
<=\$ 75,000\end{array}$ & $\begin{array}{l}35.9(31.8- \\
40.1)\end{array}$ & $39.7(35.5-44.0)$ & & $\begin{array}{l}43.5(39.3- \\
47.8)\end{array}$ & \\
\hline $\begin{array}{r}\text { Below poverty } \\
\text { level }\end{array}$ & $\begin{array}{l}13.6(10.4- \\
17.6)\end{array}$ & $30.0(25.7-34.7)$ & & $\begin{array}{l}23.7(19.9- \\
28.0)\end{array}$ & \\
\hline Unknown & $6.6(4.7-9.1)$ & $5.3(3.8-7.3)$ & & $5.8(4.1-8.0)$ & \\
\hline
\end{tabular}


Table 4. Odds ratios for HPV vaccination according to selected characteristics, Virginia, South Carolina, and Tennessee

\begin{tabular}{|c|c|c|c|c|}
\hline \multirow{3}{*}{$\begin{array}{c}\text { Characteristic } \\
\mathrm{s}\end{array}$} & \multicolumn{2}{|c|}{ Virginia \& S. Carolina } & \multicolumn{2}{|c|}{ Virginia and Tennessee } \\
\hline & $\begin{array}{c}\text { Model } 2 \\
(\mathrm{n}=2,119)\end{array}$ & $\begin{array}{l}\text { Model } 2 \\
(\mathrm{n}=957)\end{array}$ & $\begin{array}{c}\text { Model 1 } \\
(\mathrm{n}=2,119)\end{array}$ & $\begin{array}{l}\text { Model } 2 \\
(\mathrm{n}=976)\end{array}$ \\
\hline & aOR $(95 \% \mathrm{CI})$ & $\mathrm{aOR}(95 \% \mathrm{CI})$ & aOR $(95 \% \mathrm{CI})$ & aOR $(95 \% \mathrm{CI})$ \\
\hline \multicolumn{5}{|l|}{$\begin{array}{l}\text { Policy } \\
\text { implemented }\end{array}$} \\
\hline No & Ref. & Ref. & Ref. & Ref. \\
\hline Yes & $\begin{array}{c}1.95(1.23- \\
3.07)^{* *}\end{array}$ & $\begin{array}{c}3.37(0.81- \\
14.0)\end{array}$ & $\begin{array}{l}1.38(0.90- \\
2.13)\end{array}$ & $3.32(0.94-11.7)$ \\
\hline \multicolumn{5}{|l|}{ Period } \\
\hline Pre & Ref. & Ref. & Ref. & Ref. \\
\hline Post & $\begin{array}{l}2.22(1.51- \\
3.27)^{* * *}\end{array}$ & $\begin{array}{l}2.72(0.99- \\
7.47)\end{array}$ & $\begin{array}{l}1.71(1.19- \\
2.44 * *)\end{array}$ & $2.26(1.02-5.0)^{*}$ \\
\hline \multicolumn{5}{|l|}{$\begin{array}{l}\text { Interaction } \\
\text { term }\end{array}$} \\
\hline $\begin{array}{r}\text { Policy*perio } \\
\mathrm{d}\end{array}$ & $\begin{array}{c}0.75(0.44- \\
1.26)\end{array}$ & $\begin{array}{l}0.52(0.12- \\
2.23)\end{array}$ & $\begin{array}{c}0.97(0.60- \\
1.61)\end{array}$ & $0.56(0.15-2.12)$ \\
\hline \multicolumn{5}{|l|}{$\begin{array}{l}\text { vaccine } \\
\text { recommendat } \\
\text { ion }\end{array}$} \\
\hline No & & Ref. & & Ref. \\
\hline Yes & & $\begin{array}{l}10.3(6.4- \\
16.6)^{* * *}\end{array}$ & & $\begin{array}{l}9.33(6.11- \\
14.27)^{* * *}\end{array}$ \\
\hline \multicolumn{5}{|l|}{$\begin{array}{l}\text { Race/ } \\
\text { ethnicity }\end{array}$} \\
\hline White & & Ref. & & Ref. \\
\hline Hispanic & & $\begin{array}{c}0.77(0.36- \\
1.61)\end{array}$ & & $0.77(0.35-1.69)$ \\
\hline Black & & $\begin{array}{c}1.34(0.78- \\
2.34)\end{array}$ & & $0.74(0.42-1.29)$ \\
\hline Other & & $\begin{array}{l}1.57(0.71- \\
3.49)\end{array}$ & & $0.97(0.43-2.20)$ \\
\hline \multicolumn{5}{|l|}{$\begin{array}{l}\text { Maternal } \\
\text { education }\end{array}$} \\
\hline $\begin{array}{r}\text { Less than } \\
\text { high school }\end{array}$ & & Ref. & & Ref. \\
\hline High school & & $\begin{array}{l}1.21(0.48- \\
3.07)\end{array}$ & & $1.84(0.83-4.9)$ \\
\hline $\begin{array}{r}\text { Some } \\
\text { College }\end{array}$ & & $\begin{array}{c}0.76(0.29- \\
1.97)\end{array}$ & & $1.13(0.51-2.52)$ \\
\hline $\begin{array}{l}\text { college } \\
\text { graduate }\end{array}$ & & $\begin{array}{l}0.66(0.26- \\
1.71)\end{array}$ & & $1.00(0.46-2.20)$ \\
\hline
\end{tabular}




\section{Household}

income

\begin{tabular}{rcc}
\hline $\begin{array}{r}\text { Above } \\
\text { poverty } \\
>75,000\end{array}$ & Ref. & Ref. \\
\hline Above & $0.93(0.58-$ & $0.85(0.53-1.36)$ \\
poverty & $1.49)$ & \\
$<=\$ 75,000$ & $1.13(0.53-$ & $1.38(0.67-2.87)$ \\
\hline Below & $2.44)$ & $0.93(0.36-2.38)$ \\
poverty & $1.05(0.37-$ & \\
\hline Unknown & $2.95)$ & \\
\hline
\end{tabular}

No. of

medical visits

last year

\begin{tabular}{|c|c|c|}
\hline 1 to 3 & Ref. & Ref. \\
\hline 4 to 6 & $\begin{array}{c}2.04(1.24- \\
3.35)^{* *}\end{array}$ & $1.63(1.03-2.58)^{*}$ \\
\hline 7 to 9 & $\begin{array}{c}1.65(0.73- \\
3.72)\end{array}$ & $2.52(1.04-6.11)^{*}$ \\
\hline \multicolumn{3}{|l|}{$\begin{array}{l}\text { Ever been } \\
\text { uninsured } \\
\text { since age } 11\end{array}$} \\
\hline yes & Ref. & Ref. \\
\hline No & $\begin{array}{c}0.65(0.32- \\
1.31)\end{array}$ & $0.646(0.31-1.36)$ \\
\hline \multicolumn{3}{|l|}{$\begin{array}{l}\text { Mother's } \\
\text { marital status }\end{array}$} \\
\hline Married & Ref. & Ref. \\
\hline Not married & $\begin{array}{c}0.96(0.54- \\
1.71)\end{array}$ & $1.57(0.92-2.70)$ \\
\hline \multicolumn{3}{|l|}{ Teen's age } \\
\hline 13-15 years & Ref. & Ref. \\
\hline 16-17 years & $\begin{array}{c}1.07(0.71- \\
1.62)\end{array}$ & $1.21(0.812-1.79)$ \\
\hline \multicolumn{3}{|l|}{$\begin{array}{l}\text { Had 11-12- } \\
\text { year-old } \\
\text { check-up? }\end{array}$} \\
\hline Yes & Ref. & Ref. \\
\hline No & $\begin{array}{c}1.11(0.60- \\
2.07)\end{array}$ & $0.71(0.36-1.39)$ \\
\hline
\end{tabular}


Figure 2. Physician recommendation trends in Virginia, South Carolina, and Tennessee, 2008-2012

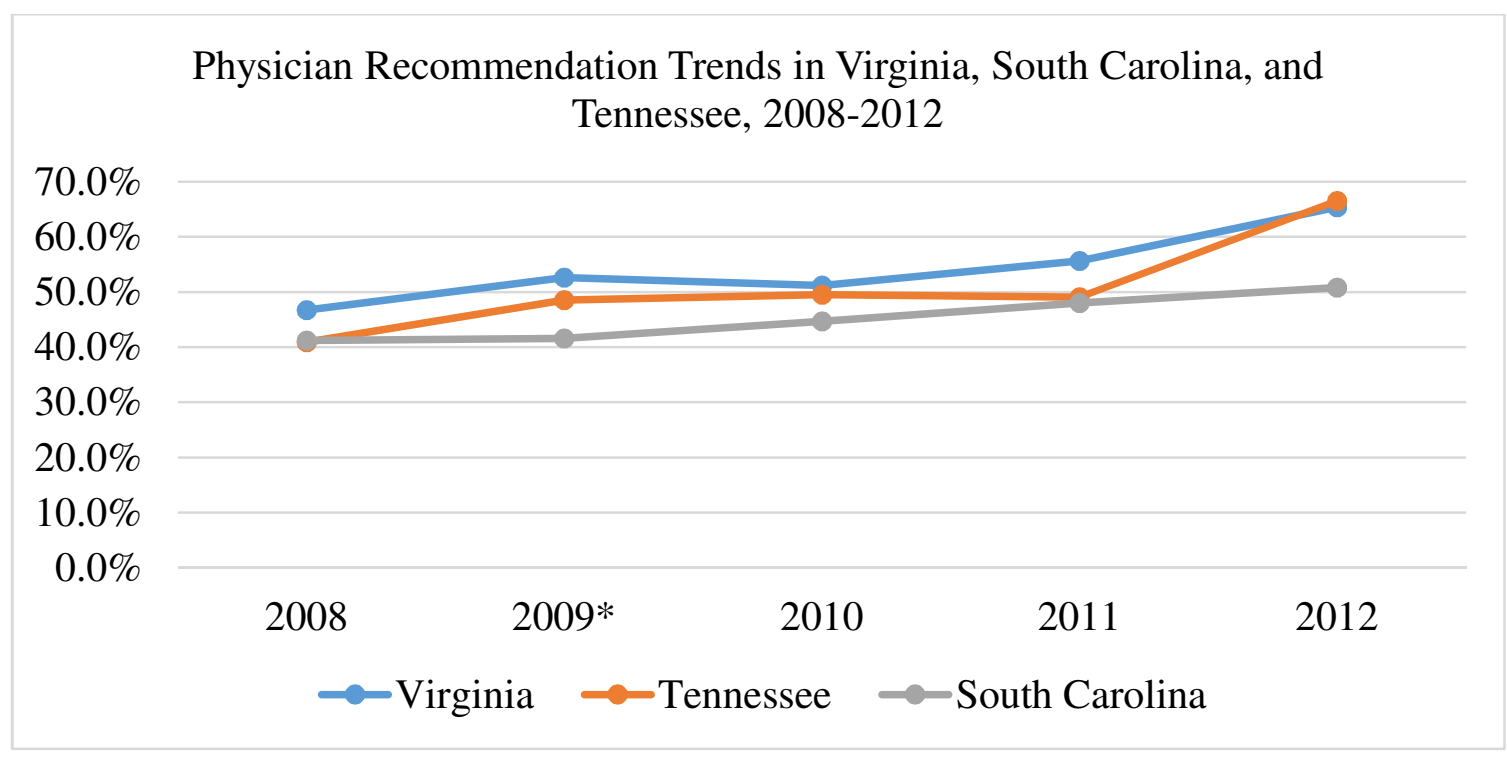

*Policy implementation year

Table 5. Difference-in-differences in HPV vaccine recommendation rates for Virginia and South Carolina, and Virginia and Tennessee

\begin{tabular}{lccc}
\hline N=2163 & $\begin{array}{c}\text { Pre-policy Period } \\
\mathbf{( 2 0 0 8 )}\end{array}$ & $\begin{array}{c}\text { Post-policy period } \\
\mathbf{( 2 0 1 0 - 1 2 )}\end{array}$ & Difference \\
\hline Virginia & 0.467 & 0.574 & 0.107 \\
\hline South Carolina & 0.412 & 0.478 & 0.066 \\
\hline Difference-in-differences & & & 0.041 \\
\hline \multicolumn{1}{c}{$\mathbf{N}=\mathbf{2 1 9 6}$} & & \\
\hline Virginia & 0.467 & 0.574 & 0.107 \\
\hline Tennessee & 0.409 & 0.551 & 0.142 \\
\hline Difference-in-differences & & & 0.035 \\
\hline$* \mathrm{p}<0.05 ; * * \mathrm{p}<0.01 ; * * * \mathrm{p}<0.001$ & &
\end{tabular}


Table 6. Odds ratios for physician recommendation according to selected characteristics, Virginia and South Carolina, and Virginia and Tennessee

\begin{tabular}{|c|c|c|c|c|}
\hline \multirow{3}{*}{$\begin{array}{l}\text { Characteristic } \\
\text { S }\end{array}$} & \multicolumn{2}{|c|}{$\begin{array}{c}\text { Virginia and South } \\
\text { Carolina }\end{array}$} & \multicolumn{2}{|c|}{ Virginia and Tennessee } \\
\hline & $\begin{array}{c}\text { Model 1 } \\
(\mathrm{N}=\mathbf{2 , 1 6 3 )}\end{array}$ & $\begin{array}{c}\text { Model } 2 \\
(\mathrm{~N}=\mathbf{1 0 0 0})\end{array}$ & $\begin{array}{c}\text { Model } 1 \\
(\mathrm{~N}=2196)\end{array}$ & $\begin{array}{l}\text { Model } 2 \\
(\mathrm{~N}=1031)\end{array}$ \\
\hline & $\mathrm{aOR}(95 \% \mathrm{CI})$ & $\begin{array}{c}\mathrm{aOR} \\
(95 \% \mathrm{CI})\end{array}$ & $\mathrm{aOR}(95 \% \mathrm{CI})$ & $\mathrm{aOR}(95 \% \mathrm{CI})$ \\
\hline \multicolumn{5}{|l|}{$\begin{array}{l}\text { Policy } \\
\text { implemented }\end{array}$} \\
\hline No & Ref. & Ref. & Ref. & Ref. \\
\hline Yes & $\begin{array}{c}1.25(0.84- \\
1.87) \\
\end{array}$ & $\begin{array}{c}1.43(0.43- \\
4.71)\end{array}$ & $1.27(0.85-1.89)$ & $0.94(0.32-2.78)$ \\
\hline \multicolumn{5}{|l|}{ Period } \\
\hline Pre & Ref. & Ref. & Ref. & Ref. \\
\hline Post & $\begin{array}{c}1.31(0.94- \\
1.81)\end{array}$ & $\begin{array}{c}1.48(0.70- \\
3.14)\end{array}$ & $\begin{array}{l}1.77(1.28- \\
2.45)^{* *}\end{array}$ & $1.18(0.58-2.37)$ \\
\hline \multicolumn{5}{|l|}{$\begin{array}{l}\text { Interaction } \\
\text { term }\end{array}$} \\
\hline Policy*period & $\begin{array}{c}1.17(0.73- \\
1.89)\end{array}$ & $\begin{array}{c}0.78(0.23- \\
2.67)\end{array}$ & $0.87(0.54-1.39)$ & $1.07(0.34-3.38)$ \\
\hline \multicolumn{5}{|l|}{$\begin{array}{l}\text { Race and } \\
\text { ethnicity }\end{array}$} \\
\hline $\begin{array}{r}\text { Non-Hispanic } \\
\text { White }\end{array}$ & & Ref. & & Ref. \\
\hline Hispanic & & $\begin{array}{c}0.78(0.33- \\
1.85)\end{array}$ & & $1.28(0.55-2.98)$ \\
\hline $\begin{array}{r}\text { Non-Hispanic } \\
\text { Black }\end{array}$ & & $\begin{array}{c}0.74(0.46- \\
1.21)\end{array}$ & & $0.79(0.48-1.29)$ \\
\hline Other & & $\begin{array}{c}0.77(0.39- \\
1.54)\end{array}$ & & $0.55(0.30-1.01)$ \\
\hline \multicolumn{5}{|l|}{$\begin{array}{l}\text { Mother's } \\
\text { education level }\end{array}$} \\
\hline $\begin{array}{r}\text { Less than high } \\
\text { school }\end{array}$ & & Ref. & & Ref. \\
\hline High school & & $\begin{array}{c}1.41(0.69- \\
2.93)\end{array}$ & & $2.24(1.09-4.61)^{*}$ \\
\hline Some College & & $\begin{array}{c}1.45(0.70- \\
3.0)\end{array}$ & & $\begin{array}{l}3.14(1.49- \\
6.59)^{* *}\end{array}$ \\
\hline $\begin{array}{l}\text { College } \\
\text { graduate }\end{array}$ & & $\begin{array}{c}1.85(0.90- \\
3.83)\end{array}$ & & $\begin{array}{c}2.87(1.34- \\
6.10) * *\end{array}$ \\
\hline
\end{tabular}




\begin{tabular}{|c|c|c|}
\hline $\begin{array}{l}\text { Household } \\
\text { income }\end{array}$ & & \\
\hline $\begin{array}{r}\text { Above poverty } \\
>\$ 75,000\end{array}$ & Ref. & Ref. \\
\hline $\begin{array}{l}\text { Above poverty } \\
<=\$ 75,000\end{array}$ & $\begin{array}{c}0.64(0.41- \\
1.01)\end{array}$ & $\begin{array}{c}0.49(0.31- \\
0.76) * *\end{array}$ \\
\hline $\begin{array}{r}\text { Below poverty } \\
\text { level }\end{array}$ & $\begin{array}{c}0.55(0.28- \\
1.08)\end{array}$ & $0.73(0.38-1.38)$ \\
\hline Unknown & $\begin{array}{l}0.33(0.14- \\
0.76)^{*}\end{array}$ & $0.60(0.25-1.45)$ \\
\hline $\begin{array}{l}\text { No. of medical } \\
\text { visits last year }\end{array}$ & & \\
\hline 1 to 3 & Ref. & Ref. \\
\hline 4 to 6 & $\begin{array}{c}1.04(0.65- \\
1.65)\end{array}$ & $1.43(0.91-2.24)$ \\
\hline 7 to 9 & $\begin{array}{l}2.36(1.09- \\
5.11)^{*}\end{array}$ & $1.79(0.25-1.45)$ \\
\hline $\begin{array}{l}\text { Ever been } \\
\text { uninsured since } \\
\text { age } 11 \text { ? }\end{array}$ & & \\
\hline yes & Ref. & Ref. \\
\hline No & $\begin{array}{c}0.33(0.17- \\
0.66) * *\end{array}$ & $0.76(0.40-1.58)$ \\
\hline $\begin{array}{l}\text { Mother's } \\
\text { marital status }\end{array}$ & & \\
\hline Married & Ref. & Ref. \\
\hline Not married & $\begin{array}{c}0.82(0.50- \\
1.34)\end{array}$ & $1.14(0.71-1.85)$ \\
\hline Teen's age & & \\
\hline $13-15$ years & Ref. & Ref. \\
\hline $16-17$ years & $\begin{array}{c}1.09(0.74- \\
1.61)\end{array}$ & $1.09(0.76-1.56)$ \\
\hline $\begin{array}{l}\text { Teen had an } \\
11-12 \text { year-old } \\
\text { check-up }\end{array}$ & & \\
\hline Yes & Ref. & Ref. \\
\hline No & $\begin{array}{c}0.62(0.33- \\
1.17)\end{array}$ & $0.96(0.50-1.83)$ \\
\hline
\end{tabular}




\title{
Manuscript 3
}

\section{Impact of Louisiana's HPV Vaccine Awareness Policy on HPV Vaccination among 13-17 Year-Old Females}

\begin{abstract}
The Advisory Committee on Immunization Practices recommends routine human papillomavirus (HPV) immunization for 11-12 year-old adolescents. In 2008, Louisiana required the school boards to distribute HPV vaccine information to parents or guardian of students in grades 6-12. This manuscript investigates the impact of this policy on HPV vaccination among 13-17 year-old female adolescents using National Immunization Survey-Teen data 2008-2012.
\end{abstract}

Drawing on the data from the 2008-2012 National Immunization Survey (NISTeen), we compared the difference in proportions of females who have been vaccinated before and after the policy $(n=2,327)$. Using difference-in-difference estimation, we explored the change in vaccination rates before and after the policy implementation in Louisiana compared to Alabama and Mississippi, who did not have such a policy in place.

The difference-in-differences estimate for HPV vaccination was not significant. Physician recommendation for HPV vaccine recommendation was strongly associated with vaccination for females in Louisiana and Alabama (aOR=7.74; 95\% CI: 5.22-11.5) and for those in Louisiana and Mississippi (aOR=7.05; 95\% CI: 4.6-10.5). Compared to the proportion of female adolescents who had received a physician recommendation in 
Alabama or Mississippi, the proportion in Louisiana did not increase significantly in the post-policy period.

HPV vaccination rates did not increase significantly in Louisiana compared to Alabama or Mississippi following the implementation of the policy. Despite Louisiana's policy, physician recommendation remains the factor most strongly associated with HPV vaccination. HPV vaccine awareness did not necessarily result in HPV vaccination.

\section{Background}

The Food and Drug Administration has licensed two HPV preventative vaccines since 2014 (U.S. Food and Drug Administration, 2014). The Advisory Committee on Immunization Practices (ACIP) recommends routine HPV immunization for 11-12 yearolds (Centers for Disease Control and Prevention, 2012). HPV vaccination rates remain well below the $80 \%$ coverage of Healthy People 2020 (U.S. Department of Health and Human Services, 2014). In 2014, only $60 \%$ of females aged 13-17 years had initiated, and $39.7 \%$ of females had completed the HPV vaccine series (Reagan-Steiner et al., 2015). Among the males in the same age-group, while $41.7 \%$ had initiated, only $21.6 \%$ had completed the vaccine series (Reagan-Steiner et al., 2015).

In March 2008, House Bill 359 (HB359) was introduced to Louisiana's House of Representatives. In June 2008, it became Act 210 without the governor's signature (Louisiana State Legislature, 2008). Act 210 requires the Department of Health and Hospitals to provide HPV/HPV vaccine information to the Department of Education, which would provide this information to the city, parish, and school boards. School boards are required to distribute the information to parents or guardian of students in 
grades 6-12. The HPV/HPV vaccine information must include a form requesting parental permission to provide HPV information to students directly (Louisiana State Legislature, 2008). When it was first introduced, Louisiana's HB359 would apply to female students in sixth grade, but it later included students of both genders in grades six through twelve (Louisiana State Legislature, 2008).

Several states have passed bills requiring HPV-related education and HPV vaccine availability for adolescents, or parents, or both. Indiana, Utah, Iowa, New Jersey, and Washington took the lead on HPV and HPV vaccine awareness legislatures in the 2006-2007 period (National Conference of States Legislatures, 2015). Since 2008, several states have considered HPV vaccine awareness policies, including Kentucky and Missouri. While several states have implemented policies aimed at increasing HPV vaccine awareness, the effectiveness of these policies at increasing HPV vaccination has not been evaluated. This study investigates the impact of Louisiana's HPV and HPV vaccine awareness policy on HPV vaccination among 13-17 year-old females using National Immunization Survey-Teen (NIS-Teen) data 2008-2012.

\section{Methods}

\section{NIS-Teen Survey}

The purpose of the National Immunization Survey (NIS) is to estimate vaccination coverage among children 19 to 35 months old. The NIS-Teen is a NIS appended survey, which uses random digit dialing telephone survey of households to provide an estimation of vaccination coverage among adolescents (Curtin, Lester et al., 
2013). NIS-Teen data collection is conducted in two stages: a household telephone survey that collects information on immunization status of adolescents from the teen's parent or guardian, along with a permission request to contact the adolescent's healthcare provider; and an immunization questionnaire mailed to teen's healthcare providers (Curtin, Lester et al., 2013). NIS-Teens' detailed survey methodology has been published elsewhere (National Centers for Health Statistics, 2013).

\section{Study Design}

The current study analyzed the effects of a natural experiment (i.e. implementation of a HPV vaccination law in Louisiana) using a pre-post implementation design (difference-in-differences), with the state of Alabama and Mississippi as comparison groups. A natural experiment is one in which an intervention varies through the natural occurrence of an event that is exogenous to the outcome of interest (Petticrew et al., 2005; Remler \& Van Ryzin, 2011). It allows comparisons between the group that experiences the event and the group that did not (Petticrew et al., 2005; Remler \& Van Ryzin, 2011). The different HPV vaccine policies implemented in the southern Black Belt states are an excellent source of natural variation.

Although several methods have been used to assess the effectiveness of public health policies, difference-in-differences is among the most widely used methods (Mason et al., 2015; Patel et al., 2014; Rajaram et al., 2014). Difference-in-Differences estimates the treatment effect on the treated group by subtracting the change in the outcome for the comparison group before and after the treatment from the change in the outcome for the treatment group before and after the treatment (Descy \& Tessaring, 2004; Meyer, 1995b). 
This approach controls for unobserved differences that may bias the treatment effect estimate (Descy \& Tessaring, 2004; Meyer, 1995b).

\section{Treatment and Comparison States}

Louisiana is located in the Black Belt, which is a part of the South. Other Black

Belt states includes Florida, Georgia, North Carolina, South Carolina, Virginia, Alabama, Mississippi, Tennessee, Arkansas, and Texas. The Black Belt is known for its high poverty, high unemployment rates, low educational attainment, high rates of health disparities, and high concentration of individuals of African descent are characteristic of this sub-region (Wimberly \& Morris, 1997). For this analysis, the control state and the comparison should be very similar except in HPV policies and other policies that may influence HPV vaccinations Black Belt states that are in geographic proximity with Louisiana include Texas, Arkansas, Mississippi and Alabama.

In 2007, Texas enacted a HPV vaccine mandate by executive order, but the mandate was later overturned by legislature. Additionally, Texas has enacted a bill requiring the Department of Health to distribute HPV-related educational materials (National Conference of States Legislatures, 2015). Therefore, Texas could not serve as a comparison state. In 2010, the Affordable Care Act was signed into law by President Obama. Initially, it would initially require the expansion of Medicaid, but the Supreme Court has made the Medicaid expansion an option for states. In the Black Belt region, only Arkansas implemented the expansion, which increased Medicaid coverage for children (Rudowitz, Artiga, \& Musumeci, 2014). As a result, Arkansas was not chosen as a comparison state for Louisiana. Neither Alabama nor Mississippi has enacted bills 
requiring the distribution of HPV-related education materials or HPV vaccinations for girls in the sixth grade.

Louisiana and Alabama are separated by the state of Mississippi. For the 20072010 period, the average median household income was $\$ 41,438$ in Louisiana, $\$ 36,697$ in Mississippi, and \$41,911 in Alabama (U.S. Census Bureau, 2013a). During the 20072012 period, the average graduation rate among 18-24 year-olds was 79\% in Louisiana, 79.4\% in Mississippi, and 82.3\% in Alabama (U.S. Census Bureau, 2014a). During the same time period, the average poverty rate was $18.7 \%$ in Louisiana, $20.9 \%$ in Mississippi, and 15.7 in Alabama (U.S. Census Bureau, 2014b). Although the high school graduation rates are more similar between Louisiana and Mississippi, the income and poverty gap is wider for Louisiana and Mississippi. Due to their similarities with Louisiana, both states were chosen as comparison states.

\section{Sample and Population}

Although Louisiana's policy became effective in June 2008 (National Conference of States Legislatures, 2015), the school parishes or boards were responsible for its implementation (Louisiana State Legislature, 2008). As a result, the implementation did not occur in the same year all parishes and school boards. The majority of parishes and school boards did not implement the policy until 2010 (Louisiana Board of Education representative, Personal Communication, March 7, 2014). The pre-policy period was 2008-2009; the implementation year was 2010; and the post-policy period was 2011-

2012. The response rate for the telephone interview was $85.2 \%$ for $2008,85.4 \%$ in 2010 , 84.7\% in 2011, and 75.5\% in 2012 (Centers for Disease Control and Prevention \& 
National Centers for Health Statistics, 2013). The sample included females aged 13-17

years whose parents or guardians responded to questions about HPV vaccination from the telephone survey. For Louisiana and Alabama 2,630 females and for Louisiana and Mississippi 2,826 females provided a response.

\section{Variables}

Maternal education, maternal marital status, household income, teen's race/ethnicity, age, gender, state of residence, health insurance status, healthcare visits in the past 12 months, and HPV vaccination status were included in the analysis. Teens who had received at least one dose were classified as vaccinated. HPV vaccination history from the telephone rather than from the healthcare provider questionnaire were used. The kappa agreement between HPV vaccination status from the household survey and HPV vaccination status from provider was $92.5 \%$.

\section{Statistical Analysis}

We performed weighted Pearson's chi-square test to investigate the variation in demographic factors between Louisiana and Alabama and between Louisiana and Alabama. Additionally, we estimated a difference-in-differences model to quantify the difference in the change in vaccination rates between Louisiana and Alabama, and between Louisiana and Mississippi before and after the policy implementation. Subsequently, a logistic regression analysis with 'policy' and 'period' interaction was performed to estimate the difference-in-differences in HPV vaccination among females living in Louisiana or Alabama in order to control for demographic variables using the following model: 
Logit $(\operatorname{Pr}(\mathrm{Y}=1$ lperiod, treatment, period*treatment, age...... physician recommendation $)=\alpha+\Upsilon_{1} *$ state $+\Upsilon_{2} *$ period $+\Upsilon_{3} *($ period $*$ state $)+\boldsymbol{\beta} \boldsymbol{X}(1)$

$\mathrm{Y}$ is HPV vaccination $(1=$ yes $/ 0=$ no), and $\boldsymbol{X}$ is a vector of control variables that included age, medical visits, maternal education, maternal income, and physician recommendation. Two logistic regression models were built to estimate difference-indifferences in HPV vaccination while controlling for demographic factors. The first model included the policy, the period, and the policy and period interaction variables. For the second model, the demographic variables were added to the first model. To estimate difference-in-differences in physician recommendation, two logistic regression models were built using the following model:

Logit $(\operatorname{Pr}(\mathrm{Y}=1$ lperiod, treatment, period*treatment, age.......and maternal income $))=\alpha+\Upsilon_{1} *$ state $+\Upsilon_{2} *$ period $+\Upsilon_{3} *($ period $*$ state $)+\boldsymbol{\beta} \boldsymbol{X}(2)$

$\mathrm{Y}$ is HPV vaccine recommendation from a physician $(1=$ yes $/ 0=$ no $)$, and $\boldsymbol{X}$ is a vector of control variables. A linktest was performed to assess the fit of the model. A pvalue $<0.05$ was used as the threshold for statistical significance. All statistical analyses were performed using STATA 13 (StataCorp, 2013), and all estimates were weighted to females aged 13-17 in the relevant states and years included in each analysis.

\section{Results}

\section{HPV Vaccination}

In 2008, 28.8\% of adolescent females in Louisiana, 25.7\% in Alabama, and 18.5\% in Mississippi received at least one dose of the HPV vaccine. Vaccination rate 
increased to 36.7\% in Louisiana, 37.7\% in Alabama, and 20.1\% in Mississippi in 2009. In 2010, vaccination rates increased to $39.9 \%$ in Louisiana, remained constant in Alabama (37.7\%), and increased to $23.2 \%$ in Mississippi. Vaccination rates increased to 50.4\% in Louisiana, 42.2\% in Alabama, and 26.5\% in Mississippi in 2011. In 2012, vaccination rates increased to $53.6 \%$ in Louisiana, $42.7 \%$ in Alabama, and $38.9 \%$ in Mississippi. While the largest increase in vaccination rates in Louisiana was from 2010 to 2011 (39.9-50.4\%), Alabama's largest increase was from 2008 to 2009 (25.7-37.7\%). For Mississippi, the greatest increase in vaccination was from 2011 to 2012 (26.5-38.9\%) (Figure 1).

\section{[Figure 1 Here]}

The difference-in-differences in vaccination rates between Louisiana and Alabama and between Louisiana and Mississippi was not significant (Table 1). In the prepolicy period, females in Louisiana and Alabama differed by insurance coverage history and household income (Table 2), whereas females in Louisiana and Mississippi differed by vaccination status, insurance coverage history, vaccine recommendation status, age at last medical check-up, and 11-12-year-old medical check-up status (Table 2). In the postpolicy period, females in Louisiana and Alabama differed only in vaccination status while females in Louisiana and Mississippi differed by vaccination status, race/ethnicity, number of medical visits, history of insurance coverage, vaccine recommendation status, age at last medical visits, and 11-12-year-old medical check-up status (Table 3). 


\section{[Table 1 Here]}

[Tables $2 \& 3$ Here]

For the logistic regression model fitting, the linktest results were not significant for any of the models, which indicated that our models ware properly specified. For females in Louisiana and Alabama, the first logistic regression model indicated that in the post-policy period, female adolescents had higher odds of taking the vaccine than in the pre-policy period ( $\mathrm{aOR}=1.60 ; 95 \% \mathrm{CI}$ : $1.16-2.6)$. The period and the interaction variables were not significant (Table 4). In the second model, HPV vaccine recommendation was strongly associated with vaccination $(\mathrm{aOR}=7.74 ; 95 \% \mathrm{CI}$ : 5.22-11.5). Black adolescent females had lower odds of being vaccinated compared to whites $(\mathrm{aOR}=0.58,95 \% \mathrm{CI}$ : 0.37-0.90). Those with family income below the federal poverty level had higher odds of being vaccinated $(\mathrm{aOR}=2.44 ; 95 \% \mathrm{CI}: 1.25-4.75)$ compared to those with a family income of $\$ 75,000$ or above. Additionally, teens who had seven or more medical visits had higher odds of vaccination $(\mathrm{aOR}=2.84 ; 95 \% \mathrm{CI}$ : $1.32-2.85)$ compared to those who had three or fewer visits (Table 4). For Louisiana and Mississippi, the first model indicates that females in Louisiana had higher odds of vaccination compared to those in Alabama (aOR=2.01; 95\%CI: 1.47-2.8) and females from both states had higher odds of vaccination in the post-policy period ( $\mathrm{aOR}=2.095 \% \mathrm{CI}: 1.4-2.77)$, but the interaction term was not significant (Table 4). In the second model, HPV vaccine recommendation was the only factor associated with vaccination ( $\mathrm{aOR}=7.05$; 95\%CI: 4.6-10.7) (Table 4). 


\section{[Table 4 Here]}

\section{Physician Recommendation}

In 2008, 38.7\% of adolescent females in Louisiana received a HPV vaccine recommendation from their healthcare provider compared to $42.5 \%$ in Alabama, and $28.5 \%$ in Mississippi (Figure 2). HPV vaccine recommendation increased to $55.8 \%$ in Louisiana, 50.0\% in Alabama, and 34.5\% in Mississippi in 2009. Vaccine recommendation decreased in all three states in 2010. The rates dropped to $44.6 \%$ in Louisiana, 49.5\% in Alabama, and 29.7\% in Mississippi. In 2011, vaccine recommendation increased to $51.9 \%$ in Louisiana, $57.1 \%$ in Alabama, and $40.8 \%$ in Mississippi. It increased to $59.5 \%$ in Louisiana, decreased to 53.1\% in Alabama, and increased to $40.8 \%$ in Mississippi in 2012. The difference-in-differences in vaccine recommendation was not significant in the Louisiana-Alabama comparison or in the Louisiana-Mississippi comparison (Table 5).

[Figure 1 Here]

[Table 5 Here]

For the logistic regression model fitting, the linktest was not significant for any the models, which indicated that our models are properly specified. For Louisiana and Alabama, the first logistic regression model estimating the difference-in-differences in physician recommendation, the odds of receiving a physician recommendation were 
higher in the post- compared to the pre-policy period ( $\mathrm{aOR}=1.44 ; 95 \% \mathrm{CI}: 1.06-1.95)$ (Table 6). The policy and the interaction variables were not significant. In the second model, females whose mother had some college education $(\mathrm{aOR}=1.95 ; 95 \% \mathrm{CI}: 1.05-$ $3.60)$ and were college graduates $(\mathrm{aOR}=2.80 ; 95 \% \mathrm{CI}: 1.47-5.35)$ had higher odds of receiving HPV vaccine recommendation. Also, adolescent females who had seven or more medical visits had lower odds of receiving physician recommendation $(\mathrm{aOR}=0.40$; 95\%CI: 0.21-0.78). Also, those who did not have their 11-12-year-old medical check-up had lower odds of receiving vaccine recommendation $(\mathrm{aOR}=0.52 ; 95 \% \mathrm{CI}: 0.30-0.90)$ (Table 6).

For Louisiana and Mississippi, the first logistic regression showed that females in Louisiana had higher odds of receiving a physician recommendation for the vaccine $(\mathrm{aOR}=1.93 ; 95 \% \mathrm{CI}: 1.45-2.56)($ Table 6$)$. Females had higher odds of receiving physician recommendation in the post- compared to the pre-policy period $(\mathrm{aOR}=1.80$; 95\% CI: 1.035-2.42). The interaction term was not significant. In the second model, females in Louisiana had higher odds of receiving the recommendation compared to those in Mississippi (aOR=1.81; 95\% CI: 1.05-3.1). The interaction term was not significant. Females whose mother had some college education $(\mathrm{aOR}=2.1 ; 95 \% \mathrm{CI}$ : $1.07-$ 4.1) had higher odds of receiving HPV vaccine recommendation. Those who did not have their 11-12-year-old medical check-up had lower odds of receiving a HPV vaccine recommendation $(\mathrm{aOR}=0.48 ; 95 \% \mathrm{CI}: 0.28-0.82)($ Table 6$)$.

[Table 6 Here] 


\section{Discussion}

\section{HPV Vaccination}

The difference-in-differences in HPV vaccination rates between Louisiana and both comparison states was not significant, suggesting that factors other than Louisiana's policy contributed to the increase in vaccination rates observed in Louisiana. The policy and period interaction term was not significant in the logistic regression models, which indicated that Louisiana's policy did not result in a significant increase in vaccination.

In agreement with previous studies, we found physician recommendation to be a major determinant of HPV vaccination (Reiter, Katz, \& Paskett, 2013; Vadaparampil et al., 2014). Additionally, in Louisiana and Alabama teens living below the poverty level had higher odds of being vaccinated, and the odds of vaccination did not differ by health insurance status since teens were 11 years of age. A previous study has reported similar vaccination rates among uninsured and insured teens (Pierre-Victor, Mukherjee, Bahelah, \& Madhivanan, 2014), and another study has reported higher vaccination rates among those living below the federal poverty level (Reagan-Steiner et al., 2015). Moreover, in Louisiana and Alabama, Black females had lower odds of being vaccinated. This finding contradicts the recent findings reported by Reagan-Steiner and colleagues (ReaganSteiner et al., 2015), but are in agreement with previous studies (Niccolai, Mehta, \& Hadler, 2011; Stokley, Dorell, \& Yankey, 2009). Lack of access to care, especially for those living in rural areas, may contribute to the lower odds of vaccination among Blacks. 


\section{Vaccine Recommendation}

The difference-in-differences in vaccine recommendation rates and vaccination rates between Louisiana and both comparison states was not significant. These findings suggest that the implementation of the policy did not lead to an increase in vaccine recommendation. HPV vaccine awareness was not captured in every survey year and therefore could not be included in the analysis. Females who did not have their 11-12year old check-up had lower odds of receiving a HPV vaccine recommendation. Adolescent females whose mother had some college education and beyond had higher odds of receiving a HPV vaccine recommendation or to recall having received a HPV vaccine recommendation even though their odds of vaccination were not significantly greater. The higher odds for physician recommendation may result from physicians assuming that mothers with a college education would be more knowledgeable about the vaccine and therefore more likely to respond positively to the recommendation for the vaccine.

\section{Socio-political Context}

Contrary to HPV vaccine mandates, vaccine awareness policies were not surrounded by controversy (Laugesen et al., 2014) nor receive negative news coverage (Casciotti et al., 2014). Even when parents and adolescents are aware of HPV vaccine, structural barriers-including lack of transportation, out-of-pocket cost, parental consent, safety concerns, and parental sexual promiscuity concerns-may hinder adolescents from taking the vaccine (Kaplan, 2010). Additionally, vaccine awareness must be followed be vaccine recommendation in order for decision-making to occur. 


\section{Limitations and Strengths}

The present study had several limitations. The major limitation of the research is the use of pooled cross-sectional data. Furthermore, difference-in-differences assumes that time-varying unmeasured characteristics are constant over the time periods in the treatment state and do not correlate with HPV vaccinations. We verified that no significant changes took place for some characteristics not measured in the dataset such as bills directly impacting Medicaid SCHIP eligibility, and Medicaid expansion under the Affordable Care Act. However, the assumption that time-varying unmeasured characteristics are constant over the time periods could not be tested fully. Additionally, this study uses pooled cross-sectional rather than longitudinal data. Moreover, while the policy and the control states are similar, they are not identical.

Despite these limitations, this study evaluated an important policy that could serve as a model for other states in the Black Belt and had several strengths. First, it used data from a nationally representative sample of non-institutionalized U.S. population. Additionally, this study is among the first few to assess the impact of Louisiana's policy requiring that schools sent HPV/HPV vaccine information to parents of students in grades 6-12 on HPV vaccination among13-17 year-old females. Furthermore, a potential pitfall in policy analysis is "policy endogeneity," which would occur, if Louisiana's policy was adopted to increase HPV vaccination due to low HPV vaccination. There was no evidence that Louisiana adopted its policy due to low HPV vaccination. 


\section{Conclusion}

The policy did not lead to HPV vaccination. Nevertheless, the importance of the delivery of uniform and accurate HPV vaccine information should not be ignored, as the information prepares parents and adolescents for the physician recommendation. Despite HPV vaccine awareness among parents, physician recommendation remains the key predictor of HPV vaccination.

Since provider recommendation plays a vital role in HPV vaccination, we must continue policy and educational efforts to not only deliver uniform and accurate HPV vaccine information in order to prepare parents and adolescents for the vaccine recommendation but also to facilitate physician recommendation. In private practice settings, the nurse and the physician assistant interact more with the patient than the physician. Therefore, they are instrumental in patient education and decision-making. They are in a position to educate the patients about HPV and the vaccine prior to their meeting with the physician and assist them in the decision-making following the physician recommendation. In other settings, healthcare providers should continue to decrease missed opportunities for HPV vaccination. Previous studies have identified school-based health centers (SBHCs) as a setting with a great potential to increase HPV vaccination (Golden et al., 2014; Kaplan, 2010), particularly for adolescents in rural areas and those who may lack access. SHBCs can boost HPV vaccinations by sending parents educational materials regarding all recommended vaccines along with immunization consent forms. 


\section{References}

Casciotti, D. M., Smith, K. C., Andon, L., Vernick, J., Tsui, A., \& Klassen, A. C. (2014). Print news coverage of school-based human papillomavirus vaccine mandates. Journal of School Health, 84(2), 71-81. doi:10.1111/josh.12126

Centers for Disease Control and Prevention. (2012). Advisory Committee on Immunization Practices: human papillomavirus vaccine resolution. Retrieved March 13, 2015, from http://www.cdc.gov/vaccines/programs/vfc/providers/resolutions.html

Centers for Disease Control and Prevention, \& National Centers for Health Statistics. (2013). National Immunization Survey-Teen A User' s Guide for the 2011. Retrieved from http://www.cdc.gov/nchs/nis/data_files_teen.htm

Curtin, Lester, R., Mohadjer, L. K., Dohrmann, S. M., Kruszan-Moran, D., Mirel, L. B., Carroll, M. D., ... Johnson, C. L. (2013). National Health and Nutrition Examination Survey: Sample design, 2007 - 2010. Vital and Health Statistics, 2(160).

Descy, P., \& Tessaring, M. (2004). Evaluation and impact of education and training: the value of learning. Retrieved from http://www.trainingvillage.gr/etv/projects_Networks/researchlab/

Golden, S. D., Moracco, K. E., Feld, A. L., Turner, K. L., DeFrank, J. T., \& Brewer, N. T. (2014). Process evaluation of an intervention to increase provision of adolescent vaccines at school health centers. Health Education \& Behavior, 41(6), 625-632. doi:10.1177/1090198114531773

Kaplan, D. W. (2010). Barriers and Potential Solutions to Increasing Immunization Rates in Adolescents. Journal of Adolescent Health, 46, S24-S33.

doi:10.1016/j.jadohealth.2009.12.004

Laugesen, M. J., Mistry, R., Carameli, K. A., Ribisl, K. M., Needleman, J., \& Bastani, R. (2014). Early policy responses to the human papillomavirus vaccine in the United States, 2006-2010. The Journal of Adolescent Health, 55(5), 659-64. doi:10.1016/j.jadohealth.2014.04.015

Louisiana State Legislature. (2008). Lousiana House Bill 359. Retrieved March 1, 2016, from http://www.legis.la.gov/legis/BillInfo.aspx?s=08RS\&b=ACT210\&sbi=y

Mason, T., Sutton, M., Whittaker, W., McSweeney, T., Millar, T., Donmall, M., ... Pierce, M. (2015). The impact of paying treatment providers for outcomes: Difference-in-differences analysis of the "payment by results for drugs recovery" pilot. Addiction, 110(7), 1120-8. doi:10.1111/add.12920

Meyer, B. D. (1995). Natural and quasi-experiments in economics. Journal of Business \& Economic Statistics, 13(2), 151-61. doi:10.2307/1392369 
National Centers for Health Statistics. (2013). Datasets for the National Immunization Survey - Teen. Retrieved November 11, 2013, from http://www.cdc.gov/nchs/nis/data_files_teen.htm

National Conference of States Legislatures. (2015). HPV Vaccine: State Legislation and Statutes. Retrieved December 24, 2015, from

http://www.ncsl.org/research/health/hpv-vaccine-state-legislation-and-statutes.aspx

Niccolai, L. M., Mehta, N. R., \& Hadler, J. L. (2011). Racial/Ethnic and poverty disparities in human papillomavirus vaccination completion. American Journal of Preventive Medicine, 41(4), 428-33. doi:10.1016/j.amepre.2011.06.032

Patel, M. S., Volpp, K. G., Small, D. S., Hill, A. S., Even-Shoshan, O., Rosenbaum, L., ... Silber, J. H. (2014). Association of the 2011 ACGME Resident Duty Hour Reforms With Mortality and Readmissions Among Hospitalized Medicare Patients. JAMA, 312(22), 2364. doi:10.1001/jama.2014.15273

Petticrew, M., Cummins, S., Ferrell, C., Findlay, A., Higgins, C., Hoy, C., ... Sparks, L. (2005). Natural experiments: an underused tool for public health? Public Health, 119(9), 751-7. doi:10.1016/j.puhe.2004.11.008

Pierre-Victor, D., Mukherjee, S., Bahelah, R., \& Madhivanan, P. (2014). Human papillomavirus vaccine uptake among males 11-26 years in United States: Findings from the national health and nutrition examination survey, 2011-2012. Vaccine, 32(49), 6655-6658. doi:10.1016/j.vaccine.2014.09.044

Rajaram, R., Chung, J. W., Jones, A. T., Cohen, M. E., Dahlke, A. R., Ko, C. Y., ... Bilimoria, K. Y. (2014). Association of the 2011 ACGME resident duty hour reform with general surgery patient outcomes and with resident examination performance. JAMA, 312(22), 2374-2384. doi:10.1001/jama.2014.15277

Reagan-Steiner, S., Yankey, D., Jeyarajah, J., Elam-Evans, L. D., Singleton, J. A., Curtis, C. R., ... Stokley, S. (2015). National, Regional, State, and Selected Local Area Vaccination Coverage among Adolescents Aged 13-17--United States, 2014. $M M W R$, 64(29), 784-792. Retrieved from http://www.ncbi.nlm.nih.gov/pubmed/26225479

Reiter, P. L., Katz, M. L., \& Paskett, E. D. (2013). Correlates of HPV vaccination among adolescent females from Appalachia and reasons why their parents do not intend to vaccinate. Vaccine, 31(31), 3121-5. doi:10.1016/j.vaccine.2013.04.068

Remler, D. K., \& Van Ryzin, G. G. (2011). Natural and quasi experiments. In Research methods in practice: Strategies for description and causation (pp. 427-464). Thousand Oaks: California: Sage. 
Rudowitz, R., Artiga, S., \& Musumeci, M. (2014). The ACA and secent section 1115 medicaid demonstration waivers. Menlo Park, CA. Retrieved from www.kff.org

StataCorp. (2013). Stata Statistical Software: Release 13. College Station, Texas: StataCorp LP.

Stokley, S., Dorell, C., \& Yankey, D. (2009). National, state, and local area vaccination coverage among adolescents aged 13-17 years- United States, 2008. MMWR, 58(36), 997-1001.

U.S. Census Bureau. (2013). Income. Retrieved April 27, 2015, from http://www.census.gov/hhes/www/income/

U.S. Census Bureau. (2014a). Educational attainment. Retrieved from httos://www.census.gov/hhes/socdemo/education/

U.S. Census Bureau. (2014b). Poverty. Retrieved April 27, 2015, from http://www.census.gov/hhes/www/poverty/

U.S. Department of Health and Human Services. (2014). Healthy People 2020. Washington, D.C. Retrieved from http://www.healthypeople.gov/2020/about/default.aspx

U.S. Food and Drug Administration. (2014). Press Announcements - FDA approves Gardasil 9 for prevention of certain cancers caused by five additional types of HPV. Retrieved June 18, 2015, from http://www.fda.gov/NewsEvents/Newsroom/PressAnnouncements/ucm426485.htm

Vadaparampil, S. T., Malo, T. L., Kahn, J. A., Salmon, D. A., Lee, J.-H., Quinn, G. P., ... Giuliano, A. R. (2014). Physicians' human papillomavirus vaccine recommendations, 2009 and 2011. American Journal of Preventive Medicine, 46(1), 80-4. doi:10.1016/j.amepre.2013.07.009

Wimberly, R. C., \& Morris, L. V. (1997). The Southern Black Belt: A national perspective. Lexington, KY: TVA Rural Studies Press. 
Figure 1. HPV vaccination' trends in Louisiana, Alabama, and Mississippi 20082012

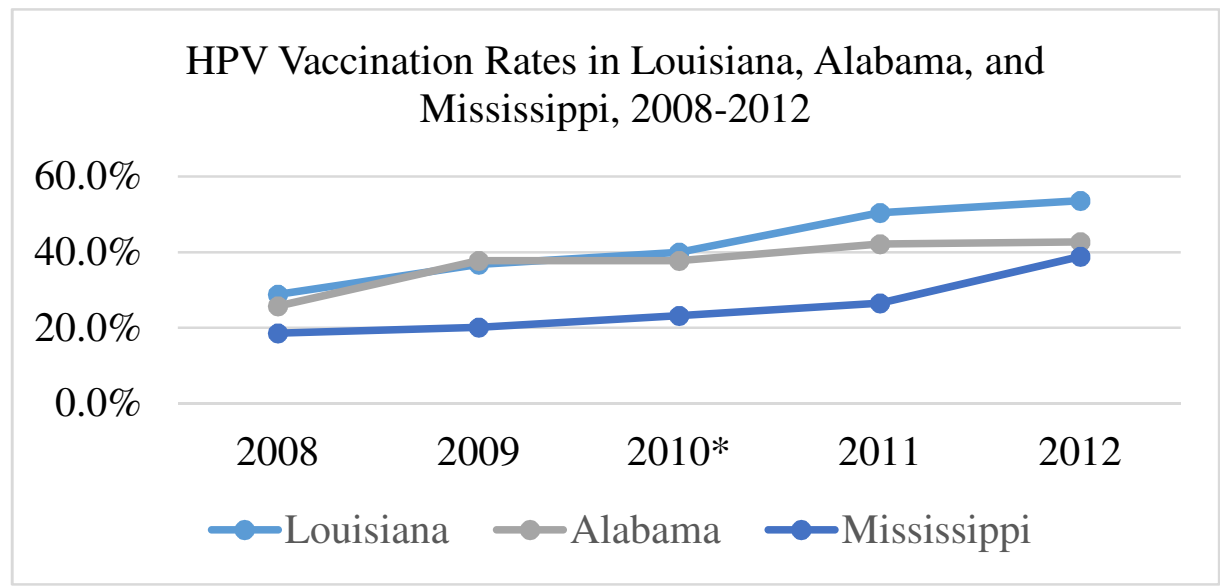

*Policy implementation year

$£ \geq 1$ dose of HPV vaccine

Table 1. Difference-in-differences in HPV vaccination rates in Louisiana and Alabama, Louisiana and Mississippi

\begin{tabular}{|c|c|c|c|}
\hline $\mathrm{N}=2,162$ & $\begin{array}{c}\text { Pre-policy Period } \\
(2008-09)\end{array}$ & $\begin{array}{c}\text { Post-policy period } \\
(2011-12)\end{array}$ & Difference \\
\hline Louisiana & 0.325 & 0.519 & $0.194 * *$ \\
\hline Alabama & 0.317 & 0.425 & 0.108 \\
\hline DiD & & & 0.086 \\
\hline \multicolumn{4}{|l|}{$\mathrm{N}=2,275$} \\
\hline Louisiana & 0.325 & 0.519 & $0.194 * *$ \\
\hline Mississippi & 0.193 & 0.323 & 0.13 \\
\hline DiD & & & 0.064 \\
\hline
\end{tabular}


Table 2. Difference in selected demographic characteristics in Louisiana and Alabama, and in Louisiana and Mississippi, pre-policy period

\begin{tabular}{|c|c|c|c|c|c|}
\hline \multirow[b]{2}{*}{ Characteristics } & $\begin{array}{c}\text { Louisiana } \\
(n=552)\end{array}$ & $\begin{array}{c}\text { Alabama } \\
(n=525)\end{array}$ & \multicolumn{3}{|c|}{$\begin{array}{l}\text { Mississippi } \\
(n=626)\end{array}$} \\
\hline & $\%(95 \% \mathrm{CI})$ & $\%(95 \% \mathrm{CI})$ & $\begin{array}{l}\text { p- } \\
\text { valu } \\
\text { e }\end{array}$ & $\%(95 \% \mathrm{CI})$ & $\begin{array}{c}\mathrm{p}- \\
\text { value }\end{array}$ \\
\hline Vaccinated & & & 0.46 & & 0.01 \\
\hline No & $\begin{array}{l}71.2(65.2- \\
76.5)\end{array}$ & $\begin{array}{l}74.3(68.0- \\
80.0)\end{array}$ & & $81.5(75.8-86.1)$ & \\
\hline Yes & $\begin{array}{l}28.8(23.5- \\
34.8)\end{array}$ & $\begin{array}{l}25.7(20.3- \\
32.0)\end{array}$ & & $\begin{array}{l}18.5(13.9- \\
24.1)\end{array}$ & \\
\hline Race and ethnicity & & & 0.19 & & 0.83 \\
\hline Hispanic & $2.1(1.0-4.1)$ & $2.6(1.1-6.0)$ & & $2.5(1.4-4.5)$ & \\
\hline Non-Hispanic White & $\begin{array}{l}52.0(45.4- \\
58.5)\end{array}$ & $\begin{array}{l}61.6(54.4- \\
68.4)\end{array}$ & & $\begin{array}{l}48.4(42.1- \\
54.8)\end{array}$ & \\
\hline Non-Hispanic Black & $\begin{array}{l}42.5(35.9- \\
49.3)\end{array}$ & $\begin{array}{l}33.4(26.8- \\
40.7)\end{array}$ & & $\begin{array}{l}45.7(39.5- \\
52.3)\end{array}$ & \\
\hline Other & $3.5(1.8-6.3)$ & $\begin{array}{l}2.4(0.93- \\
6.0)\end{array}$ & & $3.3(1.8-6.0)$ & \\
\hline Teen's age & & & 0.54 & & 0.9 \\
\hline 13-15years & $\begin{array}{l}63.2(56.9- \\
69.0)\end{array}$ & $\begin{array}{l}60.3(53.3- \\
66.9)\end{array}$ & & $\begin{array}{l}62.6(56.2- \\
68.6)\end{array}$ & \\
\hline 16-17 years & $\begin{array}{l}36.8(31.0- \\
43.2)\end{array}$ & $\begin{array}{l}39.7(33.1- \\
46.7)\end{array}$ & & $\begin{array}{l}37.4(31.4- \\
\quad 43.8)\end{array}$ & \\
\hline $\begin{array}{l}\text { No. of medical visits } \\
\text { last year }\end{array}$ & & & 0.97 & & 0.11 \\
\hline 1 to 3 & $\begin{array}{l}76.7(70.9- \\
81.6)\end{array}$ & $\begin{array}{l}76.0(69.7- \\
81.3)\end{array}$ & & $\begin{array}{l}78.4(72.5- \\
83.3)\end{array}$ & \\
\hline 4 to 6 & $\begin{array}{l}21.0(16.2- \\
26.7)\end{array}$ & $\begin{array}{l}21.8(16.7- \\
28.0)\end{array}$ & & $\begin{array}{l}16.4(12.2- \\
21.8)\end{array}$ & \\
\hline 7 to 9 & $2.3(1.2-4.5)$ & $\begin{array}{l}2.2(0.92- \\
5.1)\end{array}$ & & $5.2(2.9-9.2)$ & \\
\hline $\begin{array}{l}\text { Ever been uninsured } \\
\text { since age } 11\end{array}$ & & & $\begin{array}{c}0.00 \\
1\end{array}$ & & $\begin{array}{c}0.02 \\
2\end{array}$ \\
\hline Yes & $4.3(2.5-7.4)$ & $\begin{array}{l}13.0(8.5- \\
19.4)\end{array}$ & & $9.6(6.2-14.8)$ & \\
\hline No & $\begin{array}{l}95.7(92.6- \\
97.5)\end{array}$ & $\begin{array}{l}87.0(80.6- \\
91.5)\end{array}$ & & $\begin{array}{l}90.4(85.2- \\
93.8)\end{array}$ & \\
\hline $\begin{array}{l}\text { HPV vaccine } \\
\text { recommendation }\end{array}$ & & & 0.47 & & $\begin{array}{c}0.01 \\
9\end{array}$ \\
\hline No & $\begin{array}{l}61.3(54.9- \\
67.2)\end{array}$ & $\begin{array}{l}57.9(51.1- \\
64.4)\end{array}$ & & $\begin{array}{c}71.5(65.3- \\
77.0)\end{array}$ & \\
\hline
\end{tabular}




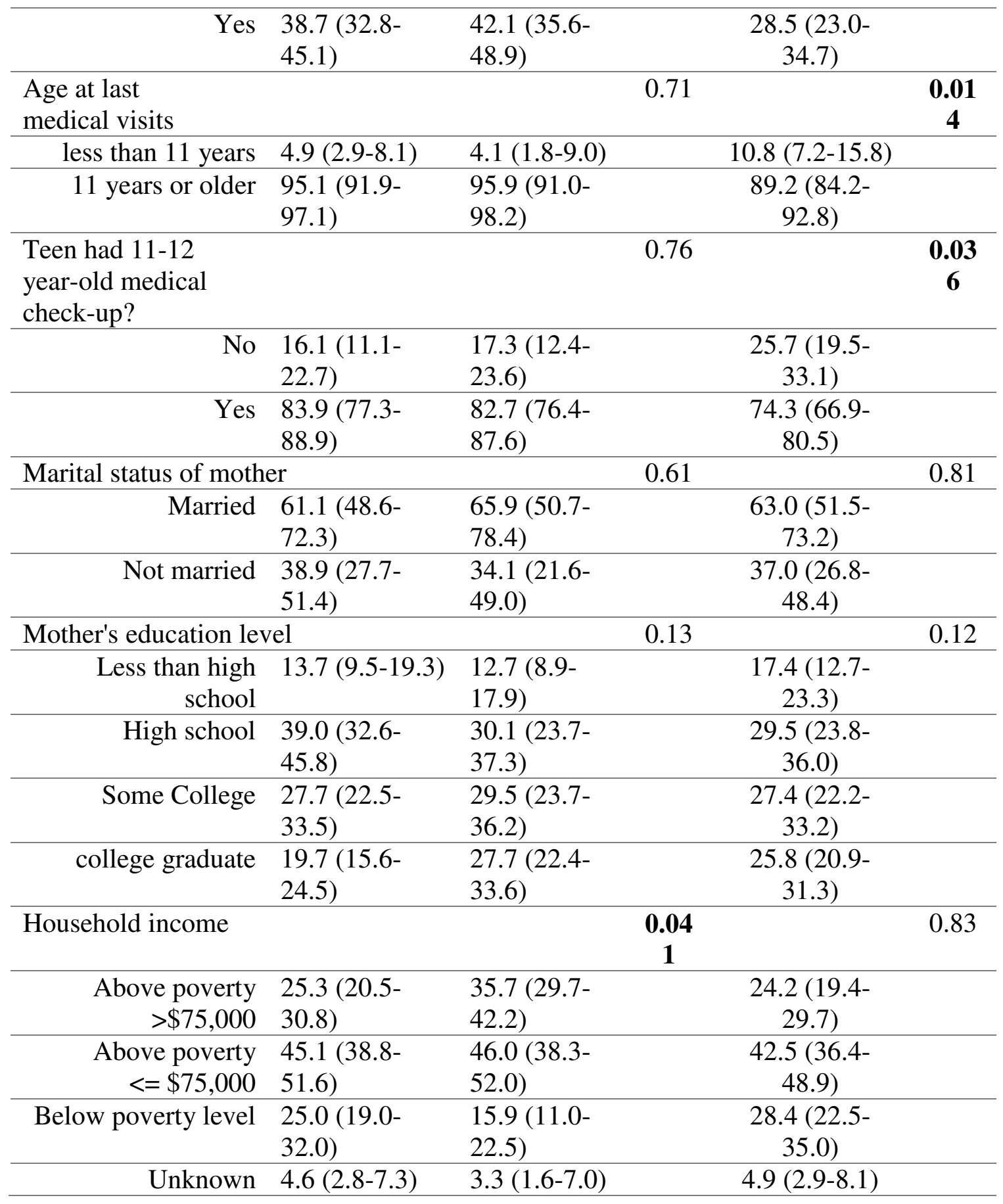


Table 3. Difference in selected demographic characteristics in Louisiana and Alabama, in Louisiana and Mississippi, post-policy period

\begin{tabular}{|c|c|c|c|c|c|}
\hline \multirow[t]{2}{*}{ Characteristics } & $\begin{array}{l}\text { Louisiana } \\
(n=598)\end{array}$ & $\begin{array}{l}\text { Alabama } \\
(n=487)\end{array}$ & \multicolumn{3}{|c|}{$\begin{array}{l}\text { Mississippi } \\
(n=529)\end{array}$} \\
\hline & $\%(95 \% \mathrm{CI})$ & $\%(95 \% \mathrm{CI})$ & $\begin{array}{c}\mathrm{p}- \\
\text { value }\end{array}$ & $\%(95 \% \mathrm{CI})$ & $\begin{array}{c}\mathrm{p}- \\
\text { value }\end{array}$ \\
\hline Vaccinated & & & 0.03 & & $\begin{array}{c}0.000 \\
1\end{array}$ \\
\hline No & $\begin{array}{c}52.4(48.1- \\
56.6)\end{array}$ & $\begin{array}{l}59.2(54.5- \\
63.7)\end{array}$ & & $70.8(66.8-74.6)$ & \\
\hline Yes & $\begin{array}{c}47.6(43.4- \\
51.9)\end{array}$ & $\begin{array}{c}40.8(36.3- \\
45.5)\end{array}$ & & $\begin{array}{c}29.2(25.4- \\
33.2)\end{array}$ & \\
\hline Race and ethnicity & & & 0.2 & & 0.006 \\
\hline Hispanic & $4.6(3.2-6.6)$ & $4.6(3.0-6.9)$ & & $2.2(1.3-3.7)$ & \\
\hline $\begin{array}{r}\text { Non-Hispanic } \\
\text { White }\end{array}$ & $\begin{array}{c}51.3(47.2- \\
55.4)\end{array}$ & $\begin{array}{l}57.7(53.1- \\
62.1)\end{array}$ & & $\begin{array}{c}48.2(44.0- \\
52.5)\end{array}$ & \\
\hline $\begin{array}{r}\text { Non-Hispanic } \\
\text { Black }\end{array}$ & $\begin{array}{c}38.5(34.4- \\
42.8)\end{array}$ & $\begin{array}{c}33.3(28.9- \\
38.0)\end{array}$ & & $\begin{array}{c}46.3(42.0- \\
50.6)\end{array}$ & \\
\hline Other & $5.5(3.9-7.8)$ & $4.5(3.2-6.2)$ & & $3.3(2.0-5.2)$ & \\
\hline Teen's age & & & 0.39 & & 0.88 \\
\hline 13-15years & $\begin{array}{c}61.3(57.2- \\
65.3)\end{array}$ & $\begin{array}{l}58.7(54.3- \\
63.0)\end{array}$ & & $\begin{array}{c}60.9(56.6- \\
64.9)\end{array}$ & \\
\hline 16-17 years & $\begin{array}{c}38.7(34.7- \\
42.8)\end{array}$ & $\begin{array}{c}41.3(37.0- \\
45.7)\end{array}$ & & $\begin{array}{l}39.1(35.1- \\
43.4)\end{array}$ & \\
\hline $\begin{array}{l}\text { No. of medical } \\
\text { visits last year }\end{array}$ & & & 0.09 & & 0.039 \\
\hline 1 to 3 & $\begin{array}{c}72.5(68.5- \\
76.1)\end{array}$ & $\begin{array}{c}75.9(72.0- \\
79.4)\end{array}$ & & $\begin{array}{c}77.6(74.0- \\
80.9)\end{array}$ & \\
\hline 4 to 6 & $\begin{array}{l}20.8(17.5- \\
24.5)\end{array}$ & $\begin{array}{l}30.2(16.9- \\
34.0)\end{array}$ & & $\begin{array}{l}18.6(15.6- \\
22.1)\end{array}$ & \\
\hline 7 to 9 & $6.7(4.8-9.2)$ & $3.9(2.7-5.5)$ & & $3.7(2.6-5.4)$ & \\
\hline $\begin{array}{l}\text { Ever been } \\
\text { uninsured since } \\
\text { age } 11\end{array}$ & & & 0.27 & & 0.02 \\
\hline Yes & $5.7(3.8-8.5)$ & $7.8(5.3-11.4)$ & & $10.4(7.6-14.0)$ & \\
\hline No & $\begin{array}{c}94.3(91.5- \\
96.2)\end{array}$ & $\begin{array}{c}92.2(88.6- \\
94.7)\end{array}$ & & $\begin{array}{c}89.6(86.0- \\
92.4)\end{array}$ & \\
\hline $\begin{array}{l}\text { HPV vaccine } \\
\text { recommendation }\end{array}$ & & & 0.68 & & $\begin{array}{c}0.000 \\
1\end{array}$ \\
\hline No & $\begin{array}{l}48.1(43.0- \\
52.3)\end{array}$ & $\begin{array}{l}46.8(42.3- \\
51.3)\end{array}$ & & $\begin{array}{c}59.9(55.7- \\
64.0)\end{array}$ & \\
\hline Yes & $\begin{array}{l}51.9(47.7- \\
56.1)\end{array}$ & $\begin{array}{l}53.2(48.7- \\
57.7)\end{array}$ & & $\begin{array}{c}40.1(36.0- \\
44.4)\end{array}$ & \\
\hline
\end{tabular}




\begin{tabular}{|c|c|c|c|c|}
\hline $\begin{array}{l}\text { Age at last } \\
\text { medical visits }\end{array}$ & & & 0.23 & $\begin{array}{c}0.000 \\
1\end{array}$ \\
\hline Less than 11 years & $3.2(2.3-4.6)$ & $4.5(3.0-6.7)$ & $8.2(6.3-10.5)$ & \\
\hline 11 years or older & $\begin{array}{c}96.8(95.4- \\
97.7)\end{array}$ & $\begin{array}{l}95.5(93.3- \\
97.5)\end{array}$ & $\begin{array}{c}91.8(89.5- \\
93.7)\end{array}$ & \\
\hline $\begin{array}{l}\text { Teen had 11-12 } \\
\text { year-old medical } \\
\text { check-up? }\end{array}$ & & & 0.24 & 0.03 \\
\hline No & $11.7(8.9-15.2)$ & $9.1(6.7-12.4)$ & $11.7(8.9-15.2)$ & \\
\hline Yes & $\begin{array}{c}88.3(84.8- \\
91.1)\end{array}$ & $\begin{array}{c}90.9(87.6- \\
93.3)\end{array}$ & $\begin{array}{c}88.3(84.8- \\
91.1)\end{array}$ & \\
\hline $\begin{array}{l}\text { Marital status of } \\
\text { mother }\end{array}$ & & & 0.78 & 0.59 \\
\hline Married & $\begin{array}{l}58.0(53.8- \\
62.1)\end{array}$ & $\begin{array}{c}58.9(54.3- \\
63.3)\end{array}$ & $\begin{array}{l}56.3(51.9- \\
60.6)\end{array}$ & \\
\hline Not married & $\begin{array}{c}42.0(37.9- \\
46.3)\end{array}$ & $\begin{array}{l}41.1(36.7- \\
45.7)\end{array}$ & $\begin{array}{c}43.7(39.4- \\
48.1)\end{array}$ & \\
\hline $\begin{array}{l}\text { Mother's } \\
\text { education level }\end{array}$ & & & 0.21 & 0.075 \\
\hline $\begin{array}{r}\text { Less than high } \\
\text { school } \\
\end{array}$ & $\begin{array}{c}14.0(11.0- \\
17.5)\end{array}$ & $\begin{array}{c}14.3(11.4- \\
17.9)\end{array}$ & $12.4(9.6-15.7)$ & \\
\hline High school & $\begin{array}{l}33.5(29.5- \\
37.8)\end{array}$ & $\begin{array}{l}28.0(23.9- \\
32.5)\end{array}$ & $\begin{array}{l}27.6(23.8- \\
31.8)\end{array}$ & \\
\hline Some College & $\begin{array}{c}25.2(22.11- \\
28.6)\end{array}$ & $\begin{array}{l}25.7(22.3- \\
29.7)\end{array}$ & $\begin{array}{l}30.8(27.0- \\
34.9)\end{array}$ & \\
\hline College graduate & $\begin{array}{c}27.3(24.1- \\
30.7)\end{array}$ & $\begin{array}{l}32.0(28.4- \\
35.9)\end{array}$ & $\begin{array}{l}29.2(25.8- \\
32.9)\end{array}$ & \\
\hline $\begin{array}{l}\text { Household } \\
\text { income }\end{array}$ & & & 0.23 & 0.051 \\
\hline $\begin{array}{r}\text { Above poverty } \\
>\$ 75,000\end{array}$ & $\begin{array}{c}28.0(24.9- \\
31.3)\end{array}$ & $\begin{array}{l}29.6(26.1- \\
33.2)\end{array}$ & $\begin{array}{l}21.9(19.1- \\
25.0)\end{array}$ & \\
\hline $\begin{array}{r}\text { Above poverty } \\
<=\$ 75,000\end{array}$ & $\begin{array}{l}39.0(35.0- \\
43.0)\end{array}$ & $\begin{array}{l}37.0(32.9- \\
41.4)\end{array}$ & $\begin{array}{l}39.2(35.1- \\
43.4)\end{array}$ & \\
\hline $\begin{array}{r}\text { Below poverty } \\
\text { level }\end{array}$ & $\begin{array}{l}28.7(24.6- \\
33.0)\end{array}$ & $\begin{array}{c}26.4(22.3- \\
30.9)\end{array}$ & $\begin{array}{l}34.6(30.3- \\
39.1)\end{array}$ & \\
\hline Unknown & $4.4(3.2-6.1)$ & $7.0(5.1-9.5)$ & $4.3(3.1-6.0)$ & \\
\hline
\end{tabular}


Table 4. Odds ratios for HPV vaccination according to selected characteristics, Louisiana and Alabama, and Louisiana and Mississippi

\begin{tabular}{|c|c|c|c|c|}
\hline \multirow{3}{*}{$\begin{array}{l}\text { Characteristi } \\
\text { cs }\end{array}$} & \multicolumn{2}{|c|}{ Louisiana and Alabama } & \multicolumn{2}{|c|}{ Louisiana and Mississippi } \\
\hline & $\begin{array}{l}\text { Model 1 } \\
(\mathrm{n}=2162)\end{array}$ & $\begin{array}{l}\text { Model } 2 \\
(n=949)\end{array}$ & $\begin{array}{c}\text { Model } 1 \\
(n=2,305)\end{array}$ & $\begin{array}{l}\text { Model } 2 \\
(n=936)\end{array}$ \\
\hline & aOR $(95 \% \mathrm{CI})$ & $\mathrm{aOR}(95 \% \mathrm{CI})$ & aOR $(95 \% \mathrm{CI})$ & aOR $(95 \% \mathrm{CI})$ \\
\hline \multicolumn{5}{|l|}{$\begin{array}{l}\text { Policy } \\
\text { implemented }\end{array}$} \\
\hline No & Ref. & Ref. & Ref. & Ref. \\
\hline Yes & $\begin{array}{c}1.04(0.77- \\
1.4)\end{array}$ & $0.80(0.44-1.4)$ & $\begin{array}{l}2.01(1.47- \\
2.8)^{* * *}\end{array}$ & $1.50(0.81-2.8)$ \\
\hline \multicolumn{5}{|l|}{ Period } \\
\hline Pre & Ref. & Ref. & Ref. & Ref. \\
\hline Post & $\begin{array}{c}1.60(1.2- \\
2.6)^{* *}\end{array}$ & $1.21(0.73-2.0)$ & $\begin{array}{l}2.0(1.4- \\
2.77) * * *\end{array}$ & $1.46(0.81-2.6)$ \\
\hline \multicolumn{5}{|l|}{$\begin{array}{l}\text { Interaction } \\
\text { term }\end{array}$} \\
\hline Policy*period & $\begin{array}{c}1.41(0.90- \\
2.2)\end{array}$ & $1.80(0.86-3.8)$ & $1.12(0.72-1.8)$ & $1.41(0.64-3.1)$ \\
\hline \multicolumn{5}{|l|}{$\begin{array}{l}\text { HPV vaccine } \\
\text { recommendati } \\
\text { on }\end{array}$} \\
\hline No & & Ref. & & Ref. \\
\hline Yes & & $\begin{array}{c}7.74(5.22- \\
15)^{* * *}\end{array}$ & & $\begin{array}{l}7.05(4.6- \\
10.7)^{* * *}\end{array}$ \\
\hline \multicolumn{5}{|l|}{$\begin{array}{l}\text { Race and } \\
\text { ethnicity }\end{array}$} \\
\hline $\begin{array}{r}\text { Non-Hispanic } \\
\text { White }\end{array}$ & & Ref. & & Ref. \\
\hline Hispanic & & $1.08(0.49-2.4)$ & & $1.37(0.45-4.1)$ \\
\hline $\begin{array}{r}\text { Non-Hispanic } \\
\text { Black }\end{array}$ & & $\begin{array}{c}0.58(0.37- \\
0.9)^{*}\end{array}$ & & $0.68(0.43-1.1)$ \\
\hline Other & & $1.21(0.50-2.9)$ & & $1.62(0.50-5.3)$ \\
\hline \multicolumn{5}{|l|}{$\begin{array}{l}\text { Mother's } \\
\text { education } \\
\text { level }\end{array}$} \\
\hline $\begin{array}{r}\text { Less than high } \\
\text { school }\end{array}$ & & Ref. & & Ref. \\
\hline High school & & $0.99(0.51-1.9)$ & & $1.13(0.55-2.3)$ \\
\hline Some College & & $1.5(0.78-2.9)$ & & $1.36(0.66-2.8)$ \\
\hline $\begin{array}{l}\text { College } \\
\text { graduate }\end{array}$ & & $1.31(0.64-2.6)$ & & $1.45(0.65-3.3)$ \\
\hline
\end{tabular}




\begin{tabular}{|c|c|c|}
\hline \multicolumn{3}{|l|}{$\begin{array}{l}\text { Household } \\
\text { income }\end{array}$} \\
\hline $\begin{array}{r}\text { Above poverty } \\
>\$ 75,000\end{array}$ & Ref. & Ref. \\
\hline $\begin{array}{r}\text { Above poverty } \\
<=\$ 75,000\end{array}$ & $1.55(0.96-2.5)$ & $1.29(0.77-2.2)$ \\
\hline $\begin{array}{r}\text { Below poverty } \\
\text { level }\end{array}$ & $\begin{array}{c}2.44(1.25- \\
4.8)^{* *}\end{array}$ & $1.72(0.78-3.8)$ \\
\hline Unknown & $1.23(0.53-2.9)$ & $2.6(0.91-5.6)$ \\
\hline \multicolumn{3}{|l|}{$\begin{array}{l}\text { No. of medical } \\
\text { visits last year }\end{array}$} \\
\hline 1 to 3 & Ref. & Ref. \\
\hline 4 to 6 & $1.39(0.90-2.1)$ & $1.30(0.80-2.1)$ \\
\hline 7 to 9 & $\begin{array}{l}2.84(1.32- \\
2.9)^{* *}\end{array}$ & $2.24(0.97-5.2)$ \\
\hline \multicolumn{3}{|l|}{$\begin{array}{l}\text { Ever been } \\
\text { uninsured } \\
\text { since age } 11\end{array}$} \\
\hline Yes & Ref. & Ref. \\
\hline No & $1.41(0.75-2.7)$ & $1.64(0.78-3.4)$ \\
\hline \multicolumn{3}{|l|}{$\begin{array}{l}\text { Mother's } \\
\text { marital status }\end{array}$} \\
\hline Married & Ref. & Ref. \\
\hline Not married & $1.26(0.82-1.9)$ & $1.11(0.71-1.7)$ \\
\hline \multicolumn{3}{|l|}{ Teen's age } \\
\hline $13-15$ years & Ref. & Ref. \\
\hline $16-17$ years & $1.08(0.76-1.5)$ & $0.95(0.65-1.4)$ \\
\hline \multicolumn{3}{|l|}{$\begin{array}{l}\text { Teen had } 11- \\
12 \text { year-old } \\
\text { check-up? }\end{array}$} \\
\hline Yes & Ref. & Ref. \\
\hline No & $0.91(0.51-1.6)$ & $1.2(0.64-2.2)$ \\
\hline
\end{tabular}


Figure 4. Physician recommendation trends in Louisiana and Alabama, and Mississippi, 2008-2012

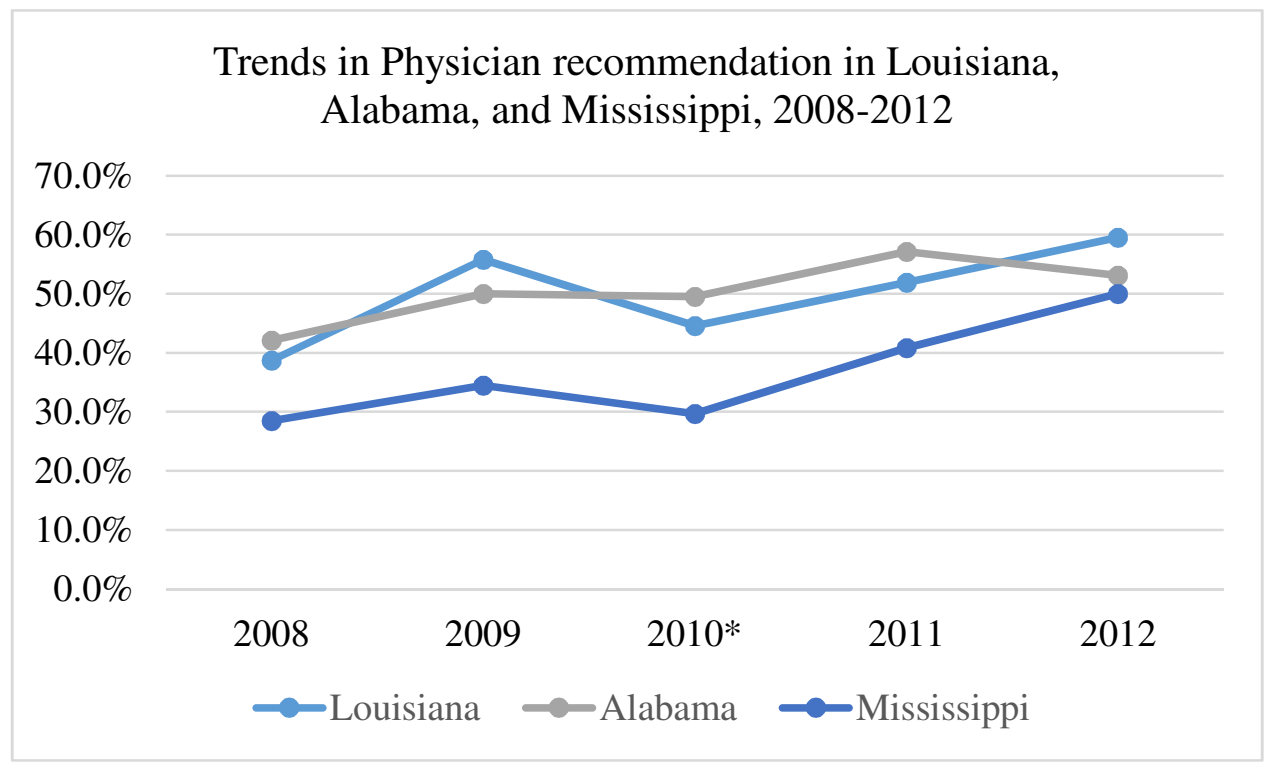

*Policy implementation year

Table 5. Difference-in-differences estimates for physician recommendation proportions in Louisiana and Alabama, Louisiana and Mississippi

\begin{tabular}{cccc}
\hline N= 2,218 & $\begin{array}{c}\text { Pre-policy period } \\
(\mathbf{2 0 0 8 - 0 9 )}\end{array}$ & $\begin{array}{c}\text { Post-policy period } \\
\mathbf{( 2 0 1 1 - 1 2 )}\end{array}$ & Difference \\
\hline Louisiana & 0.47 & 0.557 & 0.087 \\
\hline Alabama & 0.461 & 0.551 & 0.09 \\
\hline DiD & & & 0.003
\end{tabular}

\begin{tabular}{cccc}
\hline $\mathbf{N = 2 , 3 4 3}$ & & & \\
\hline Louisiana & 0.47 & 0.557 & $0.087 * * *$ \\
\hline Mississippi & 0.315 & 0.454 & $0.139 * * *$ \\
\hline DiD & & & 0.052
\end{tabular}

$* \mathrm{p}<0.05 ; * * \mathrm{p}<0.01 ; * * * \mathrm{p}<0.001$

$\mathrm{DiD}=$ difference-in-differences 
Table 6. Odds ratios for HPV vaccine recommendation according to selected characteristics, Louisiana and Alabama, Louisiana and Mississippi

\begin{tabular}{|c|c|c|c|c|}
\hline \multirow{3}{*}{ Characteristics } & \multicolumn{2}{|c|}{ Louisiana and Alabama } & \multicolumn{2}{|c|}{ Louisiana and Mississippi } \\
\hline & $\begin{array}{c}\text { Model 1 } \\
(\mathrm{N}=2,227)\end{array}$ & $\begin{array}{l}\text { Model 2( } \\
\mathrm{N}=1,007)\end{array}$ & $\begin{array}{c}\text { Model 1 } \\
(\mathrm{N}=2,375)\end{array}$ & $\begin{array}{l}\text { Model } 2 \\
(\mathrm{~N}=998)\end{array}$ \\
\hline & $\begin{array}{c}\mathrm{aOR} \\
(95 \% \mathrm{CI})\end{array}$ & $\begin{array}{c}\mathrm{aOR} \\
(95 \% \mathrm{CI})\end{array}$ & $\begin{array}{c}\mathrm{aOR} \\
(95 \% \mathrm{CI})\end{array}$ & $\begin{array}{c}\mathrm{aOR} \\
(95 \% \mathrm{CI})\end{array}$ \\
\hline \multicolumn{5}{|l|}{ Policy implemented } \\
\hline No & Ref. & Ref. & Ref. & Ref. \\
\hline Yes & $\begin{array}{c}1.04(0.78- \\
1.38)\end{array}$ & $\begin{array}{c}1.11(0.66- \\
1.87)\end{array}$ & $\begin{array}{c}1.93(1.45- \\
2.6)^{* * *}\end{array}$ & $\begin{array}{l}1.81(1.05- \\
3.1)^{*}\end{array}$ \\
\hline \multicolumn{5}{|l|}{ Period } \\
\hline Pre & Ref. & Ref. & Ref. & Ref. \\
\hline Post & $\begin{array}{c}1.44(1.06- \\
1.95)^{*}\end{array}$ & $\begin{array}{c}1.23(0.76- \\
2.0)\end{array}$ & $\begin{array}{l}1.80(1.35- \\
2.4)^{* * * *}\end{array}$ & $\begin{array}{c}1.34(0.81- \\
2.2)\end{array}$ \\
\hline \multicolumn{5}{|l|}{ Interaction term } \\
\hline Policy*period & $\begin{array}{c}0.99(0.64- \\
1.51)\end{array}$ & $\begin{array}{c}1.09(0.56- \\
2.1)\end{array}$ & $\begin{array}{c}0.79(0.52- \\
1.2)\end{array}$ & $\begin{array}{c}0.99(0.49- \\
2.0)\end{array}$ \\
\hline \multicolumn{5}{|l|}{ Race and ethnicity } \\
\hline Non-Hispanic White & & Ref. & & Ref. \\
\hline Hispanic & & $\begin{array}{c}1.71(0.71- \\
4.2)\end{array}$ & & $\begin{array}{c}1.10(0.40- \\
3.0)\end{array}$ \\
\hline Non-Hispanic Black & & $\begin{array}{c}1.05(0.42- \\
2.6)\end{array}$ & & $\begin{array}{c}0.77(0.27- \\
1.3)\end{array}$ \\
\hline Other & & $\begin{array}{c}1.49(0.50- \\
4.4)\end{array}$ & & $\begin{array}{c}1.09(0.30- \\
4.0)\end{array}$ \\
\hline \multicolumn{5}{|l|}{$\begin{array}{l}\text { Mother's education } \\
\text { level }\end{array}$} \\
\hline Less than high school & & Ref. & & Ref. \\
\hline High school & & $\begin{array}{c}1.17(0.64- \\
2.1)\end{array}$ & & $\begin{array}{c}1.13(0.58- \\
2.2)\end{array}$ \\
\hline Some College & & $\begin{array}{c}1.95(1.06- \\
3.6)^{*}\end{array}$ & & $\begin{array}{l}2.1(1.07- \\
4.1)^{*}\end{array}$ \\
\hline College graduate & & $\begin{array}{c}2.80(1.47- \\
5.4)^{* *}\end{array}$ & & $\begin{array}{l}2.3(1.15- \\
4.6)\end{array}$ \\
\hline \multicolumn{5}{|l|}{ Household income } \\
\hline $\begin{array}{r}\text { Above poverty } \\
>\$ 75,000\end{array}$ & & Ref. & & Ref. \\
\hline $\begin{array}{r}\text { Above poverty } \\
<=\$ 75,000\end{array}$ & & $\begin{array}{c}0.92(0.60- \\
1.4)\end{array}$ & & $\begin{array}{c}0.90(0.59- \\
1.4)\end{array}$ \\
\hline Below poverty level & & $\begin{array}{c}1.00(0.56- \\
1.8)\end{array}$ & & $\begin{array}{c}0.76(0.42- \\
1.4)\end{array}$ \\
\hline
\end{tabular}




\begin{tabular}{lcc}
\hline Unknown & $0.99(0.44-$ & $1.44(0.58-$ \\
& $2.2)$ & $3.5)$ \\
\hline $\begin{array}{l}\text { No. of medical visits } \\
\text { last year }\end{array}$ & & \\
\hline & & Ref. \\
\hline 4 to 3 & $1.05(0.71-$ & $1.12(0.73-$ \\
& $1.6)$ & $1.8)$ \\
\hline 7 to 9 & $0.40(0.21-$ & $0.70(0.35-$ \\
& $0.78)^{* *}$ & $1.4)$ \\
\hline
\end{tabular}

Ever been uninsured since age 11 ?

\begin{tabular}{|c|c|c|}
\hline Yes & Ref. & Ref. \\
\hline No & $\begin{array}{c}1.17(0.64- \\
1.6)\end{array}$ & $\begin{array}{c}0.93(0.49- \\
1.8)\end{array}$ \\
\hline \multicolumn{3}{|l|}{ Mother's marital status } \\
\hline Married & Ref. & Ref. \\
\hline Not married & $\begin{array}{c}1.22(0.82- \\
1.8)\end{array}$ & $\begin{array}{c}1.19(0.78- \\
1.8)\end{array}$ \\
\hline \multicolumn{3}{|l|}{ Teen's age } \\
\hline 13-15 years & Ref. & Ref. \\
\hline 16-17 years & $\begin{array}{c}1.04(0.75- \\
1.5)\end{array}$ & $\begin{array}{c}1.02(0.71- \\
1.5)\end{array}$ \\
\hline \multicolumn{3}{|l|}{$\begin{array}{l}\text { Teen had an 11-12 } \\
\text { year-old check-up? }\end{array}$} \\
\hline Yes & Ref. & Ref. \\
\hline No & $\begin{array}{c}0.52(0.30- \\
0.90)^{*}\end{array}$ & $\begin{array}{c}0.48(0.28- \\
0.8)^{* *}\end{array}$ \\
\hline
\end{tabular}

$* \mathrm{p}<0.05 ; * * \mathrm{p}<0.01 ; * * * \mathrm{p}<0.001$ 


\section{Conclusion}

This dissertation sought to provide an estimation of HPV infection in the South as compared to the rest of the country to highlight the geographic variation in the United States. It was hypothesized that the prevalence of infection from oncogenic HPV types would be higher in the South compared to the rest of the country. Although this hypothesis was not true for females aged 14-26 years, it was true for females 27-59 years old. Moreover, this dissertation sought to evaluate two HPV vaccination policies that could serve as prototypes for Southern states. It was hypothesized that HPV vaccination rates would be significantly higher in the period after the policy was implemented compared with the period preceding the implementation of both Virginia's and Louisiana's policy. Findings indicated that neither of the two policies have resulted in an increase in vaccination rates.

Several policy analysis frameworks were considered for the theoretical framework of the study. Since the study included a problem identification and a policy evaluation components, Lasswell's Stages Heuristic Public Policy Framework (Lasswell, 1956) was selected. Lasswell's theory is among the most well-known public policy theories. He identified four stages in the public policy process: agenda setting, formulation, implementation, and evaluation. The agenda setting is the stage in which a social problem issue is identified. In the formulation stage, legislators or policy-makers design, introduce, and enact a policy to resolve the social problem previously identified. In the implementation stage, the policies are carried out, and in the evaluation stage, the impact of the policy is assessed (Lasswell, 1956). Lasswell's Stages Heuristic Public 
Policy theory presumes a linear process, thereby oversimplifying the complexity of public policy process. This theory has been widely criticized, particularly for its presumption of a linear policy process (Sabatier, 2007). Nonetheless, the Lasswell's Stages Heuristic approach encompasses the entire public policy process, which provides a wide framework to situate this research.

The conceptual framework of this dissertation was founded upon the Linking Health-related Policy to Health Systems and Outcomes framework (Hardee, Irani, Maclnnis, \& Hamilton, 2012). The Linking Health-Related Policy to Health Systems and Outcomes framework was based on Lasswell's Stages Heuristic theory and included the four stages. Linking Health-Related Policy to Health Systems and Outcomes includes seven principal components: enabling environment, health-related policy development, health policy and program implementation, health systems and health outcomes, policy monitoring, program monitoring, policy and program evaluation (Hardee et al., 2012).

Linking Health-Related Policy to Health Systems and Outcomes framework considers an enabling environment that includes two aspects. The first aspect of the enabling environment includes factors such as political stability, government effectiveness, and accountability. The second aspect includes political, socio-cultural, and economic factors that may facilitate or hinder the policy implementation. It is in such an enabling environment that effective public policies can be developed and implemented because it dictates not only the development and implementation, but also the impact of the policy. The influence of the social, political, and economic factors on the implementation and effectiveness of the policy were considered. Additionally, the model considers the 
problem identification and the health outcome that the policy is intended to influence (Hardee et al., 2012). Pertaining to this study, several cancers result from HPV infection, but for Southern states, cervical cancer disparities was the central health concern guiding HPV vaccination policies. Furthermore, Hardy and colleagues pointed out that the evaluation may quantify the adoption of a health behavior by the intended population (Hardee et al., 2012). Relating to this study, HPV vaccination is the healthy behavior whose adoption was quantified.

The dissertation had a few limitations. The first limitation is the use of selfreported data in all three manuscripts. Self-reported data are subject to recall bias. This bias may have decreased the internal validity of the study. The data used for the dissertation were also subject to social desirability bias, particularly for sexual risk behaviors. Sexual behavior variables were collected using ACASI questionnaires rather than face-to-face interviews. ACASI minimizes social desirability bias. The residual desirability bias may have resulted in an underrepresentation of sexual risk behaviors, thereby reducing study internal validity. Regarding the policy analysis component, although the comparison states share regional characteristics with the policy or treatment states, they were not identical. Moreover, the collection of some variables previously included the NIS-Teen discontinued after 2009. As a result, they could not be included in the policy analysis. One such variable was HPV or HPV vaccine knowledge. Had this variable been collected for all survey years included in the analysis, the change in HPV or HPV vaccine awareness could have been assessed for the treatment or policy states. 


\section{Major Findings}

Despite these limitations, the dissertation filled several important gaps in the literature and produced the following findings:

- The prevalence of infection from high-risk HPV types was higher among females aged 27-59 years in the South compared to their counterparts in the rest of the country.

○ The prevalence infection with oncogenic HPV types was higher among females aged 27-59 years in the South compared to their counterparts in the rest of the country.

- Having more than four lifetime sex partners was associated with HPV infections.

- Women who were sexually active and unmarried had higher odds of HPV infections compared with those who were married.

- Virginia's HPV vaccine mandate for school-entry may not have resulted in a significant increase in HPV vaccination among adolescent females.

- In Virginia, physician recommendation remained strongly associated with HPV vaccination in spite of the implementation of the mandate.

○ Louisiana's HPV/HPV vaccinate awareness policy may not have led to a significant increase in HPV vaccination when compared with states without such a policy.

○ Physician recommendation was strongly associated with HPV vaccination in Louisiana in spite of the parental awareness policy. 


\section{Public Health and Policy Implications}

Despite the availability of cervical cancer screening in the United States, the South continues to experience cervical cancer disparities. HPV infection from high-risk types are higher in the South among women aged 27 years and above. To reduce disparities in cervical cancer incidence and mortality, public health efforts to increase cervical cancer screening in the South must continue. We must not only strive to achieve Healthy People 2020's projected screening goal of 93\%, but we must also ensure that screening services are accessible to women who are least likely to be screened.

Particularly among Southern women, cervical cancer screening services should be made accessible to those with less than high school education, those living at $200 \%$ or below the federal poverty level (National Institute of Health, 2015), and those living in rural areas (Doescher \& Jackson, 2009). Additionally, the rates of HPV infection are relatively high among 14-26 year-old females, which highlights the importance of vaccinating preteens against HPV.

The policy impact evaluation component of the dissertation was intended to facilitate evidence-based HPV vaccination policy-making. The effectiveness of a health policy depends generally on sociocultural, political, and economic factors, among others. HPV vaccination policies are not exceptional. The public perception of a policy, which depends, in part, on the media coverage, may have a considerable influence on its acceptance. In the case of Virginia's HPV vaccine mandate for school-entry, the media coverage was not consistent or generally positive. Additionally, parents perceived the 
mandate as an infringement on their rights. Such an unfavorable environment likely reduced parental compliance to the mandate.

HPV vaccine awareness policies did not receive negative media coverage. However, HPV vaccination awareness alone was insufficient to lead to HPV vaccination. There are several factors that create a chasm between parental HPV vaccine awareness and getting the adolescent vaccinated. These barriers include lack of transportation, outof-pocket cost, parental consent, safety concerns, and parental concerns that the vaccine will promote sexual promiscuity. Uniform and consistent HPV information should be delivered to parents in order for them to understand the reasons the vaccine is needed. Furthermore, HPV vaccine awareness prepares parents and adolescents for physician recommendation and to ask pertinent questions to healthcare providers. Therefore, HPV vaccine awareness policies should be encouraged.

Physician recommendation remains the principal factor associated with HPV vaccination. As a result, policy-makers should consider policies that may increase physician recommendation. State should introduce bills to fund HPV education and best practices regarding vaccine recommendation for current and future physicians. Additionally, similar trainings should be funded for and provided to other medium-level healthcare providers such as nurse practitioners and physician assistants, particularly those practicing in areas with cervical cancer disparities. 


\section{Directions for Future Research}

The findings indicate a higher prevalence of HPV infection from high-risk types in the American South. Further studies are needed to help understand and eliminate barriers to cervical cancer screening in the South. We did not find evidence that Virginia's vaccine mandate or Louisiana's HPV education policy led to an increase in vaccination among females aged 13-17 years. However, it found a strong association between physician recommendation and vaccination. Despite these important findings, the research could not point out all the factors that could dilute the impact of Louisiana's HPV vaccination awareness or Virginia's HPV vaccine mandate for school-entry. Investigations focusing on parental views as well as physician's views of state policies are needed to better understand the socio-cultural contexts in which these policies may be effective. Moreover, since physician recommendation is the factor most strongly associated with vaccination, efforts to encourage physician recommendation must continue along with research to better understand its facilitators. Furthermore, additional studies should investigate the role of nurses and physician assistants in facilitating physician recommendation.

The dissertation supplied evidence coherent with the higher rates of cervical cancer in the American South. Furthermore, it has evaluated the impact of a HPV awareness policy as well as a HPV vaccine mandate. Although findings of this research indicated a higher prevalence of high-risk oncogenic HPV types among Southern women aged 27-59, it could not elucidate the causes for the geographic disparities in HPV infection. Further research may be needed to understand whether causal factors for these 
differences are modifiable. Moreover, the research could not point out all the factors that hindered the effectiveness of Louisiana's HPV vaccination awareness or Virginia's mandate. Investigations focusing on parental views as well as physician's views of their state policies are needed to better understand the socio-cultural contexts in which these policies may be effective.

\section{References}

Doescher, M. P., \& Jackson, J. E. (2009). Trends in Cervical and Breast Cancer Screening Practices Among Women in Rural and Urban Areas of the United States. Journal of Public Health Management Practice, 15(3), 200-209.

Hardee, K., Irani, L., Maclnnis, R., \& Hamilton, M. (2012). Linking Health Policy With Systems and Health Outcomes: A conceptual Framework. Wahsington, D.C. Retrieved from http://www.healthpolicyproject.com/index.cfm?id=publications\&get=pubID\&pubId $=186$

Lasswell, H. D. (1956). The Decision Process. College park, MD: University of Maryland Press.

National Institute of Health. (2015). Cancer trends progress report: cervical cancer screening. Retrieved April 8, 2016, from http://progressreport.cancer.gov/detection/cervical_cancer

Sabatier, P. A. (Ed.). (2007). Theories of the Policy Process (2nd ed.). Boulder, CO: Westview Press. 
VITA

\section{DUDITH PIERRE-VICTOR}

Born, Terrier-Rouge, Northeast Department, Haiti

\begin{tabular}{|c|c|}
\hline 2003-2006 & Associate in Arts (A.A.) \\
\hline & Palm Beach State College \\
\hline & Lake Worth, Florida \\
\hline $2006-2008$ & Bachelor of Science (B.S.) \\
\hline & University of South Florida \\
\hline & Tampa, Florida \\
\hline 2009-2011 & Master of Public Health (MPH) \\
\hline & Florida International University \\
\hline & Miami, Florida \\
\hline 2012-2014 & Doctoral Student \\
\hline & Florida International University \\
\hline & Miami, Florida \\
\hline 2012-2015 & McKnight Doctoral Fellowship \\
\hline & The Florida Education Fund \\
\hline & Tampa, Florida \\
\hline 2015-2016 & Dissertation Year Fellowship \\
\hline & University Graduate School \\
\hline & Florida International University \\
\hline & Miami, Florida \\
\hline 2014-present & Doctoral Candidate \\
\hline & Florida International University \\
\hline & Miami, Florida \\
\hline
\end{tabular}




\section{PUBLICATIONS AND PRESENTATIONS (Selected)}

Madhivanan, P., Barreto, G.A., Revawala, A., Anderson, C., McKinney, S., \& PierreVictor, D. (2013). Where are we with partner treatment in BV? A critical appraisal of the latest systematic review. Sex Transm Dis. 40(6):518.

Mukherjee, S., Pierre-Victor, D., Bahelah, R., \& Madhivanan, P. (2014). Mental Health issues among Pregnant Women in Correctional Facilities: A systematic review. Women and Health, 54(8), 816-842.

Pierre-Victor, D., Mukherjee, S., Bahelah, R., \& Madhivanan, P. (2014). Human Papillomavirus Vaccine Uptake among Males 11-26 Years in United States: Findings from the National Health and Nutrition Examination Survey, 2011-2012. Vaccine, 32(49), 6655-6658.

Mukherjee, S., Trepka, M., Pierre-Victor, D., Bahelah, R., \& Avent, T. (2016). Racial/Ethnic Disparities in Antenatal Depression in the United States: A Systematic Review. Maternal and Child Health Journal, 2016 Mar 25. doi: 10.1007/s10995-0161989-x [Epub ahead of print]

Madhivanan, P., Pierre-Victor, D., Mukherjee, S.,Bhoite, P., Powell, B., Jean-Baptiste, N., Clarke, R., Avent T., Krupp, K., Human Papillomavirus Vaccination and Sexual Disinhibition in Females: A Systematic Review. American Journal of Preventive Medicine, 2016 April 26. doi: 10.1016/j.amepre.2016.03.015. [Epub ahead of print]

Pierre-Victor, D., Mukherjee S., \& Bahelah, R. \& Madhivanan, P. (2014, June). Risk Compensation Following Sexually Transmitted Infections Prevention Interventions: A Systematic Review. Poster presentation at the $47^{\text {th }}$ Annual SER Meeting, Seattle, WA.

Mukherjee, S., Pierre-Victor, D., Bahelah, R., \& Trepka, M. J. (2014, November). Racial/ethnic disparities in antenatal depression in the United States: A systematic review. Poster presentation at the $142^{\text {nd }}$ APHA Annual Meeting; New Orleans, LA.

Pierre-Victor, D., Antoine, R., Clarke, R., Madhivanan, P. (2014, November). Factors influencing human papillomavirus vaccine decision among young Haitian women. Poster presentation at the $142^{\text {nd }}$ APHA Annual Meeting; New Orleans, LA.

Pierre-Victor, D., Clarke, R., Jean-Baptiste, N., Bahelah, R., Mukherjee, S., Anderson, C., Avent, T., Nebhrajani, R., Zohourian, T., \& Madhivanan, P. (2014, April). Systematic review of risk compensation following HPV vaccination. Poster Presentation at the Herbert Wertheim College of Medicine Symposium, Miami, FL. 